ANALYSIS \& PDE Volume $5 \quad$ No. $4 \quad 2012$ MATTHIEU HILLAIRET AND PIERRE RAPHAËL SMOOTH TYPE II BLOW-UP SOLUTIONS TO THE FOUR-DIMENSIONAL ENERGY-CRITICAL WAVE EQUATION 


\title{
SMOOTH TYPE II BLOW-UP SOLUTIONS TO THE FOUR-DIMENSIONAL ENERGY-CRITICAL WAVE EQUATION
}

\author{
MATthieu Hillairet AND PIERRE RAPHAËL
}

We exhibit $\mathscr{C}^{\infty}$ type II blow-up solutions to the focusing energy-critical wave equation in dimension $N=4$. These solutions admit near blow-up time a decomposition

$$
u(t, x)=\frac{1}{\lambda^{(N-2) / 2}(t)}(Q+\varepsilon(t))\left(\frac{x}{\lambda(t)}\right), \quad \text { with }\left\|\varepsilon(t), \partial_{t} \varepsilon(t)\right\|_{\dot{H}^{1} \times L^{2}} \ll 1,
$$

where $Q$ is the extremizing profile of the Sobolev embedding $\dot{H}^{1} \rightarrow L^{2^{*}}$, and a blow-up speed

$$
\lambda(t)=(T-t) e^{-\sqrt{|\log (T-t)|}(1+o(1))} \quad \text { as } t \rightarrow T .
$$

\section{Introduction}

Setting of the problem. We deal in this paper with the energy-critical focusing wave equation

$$
\begin{cases}\partial_{t t} u-\Delta u-f(u)=0 & \text { with } f(t)=t^{(N+2) /(N-2)}, \\ \left(u, \partial_{t} u\right)_{\mid t=0}=\left(u_{0}, u_{1}\right), & (t, x) \in \mathbb{R} \times \mathbb{R}^{N}\end{cases}
$$

in dimension $N=4$. This is a special case of the nonlinear wave equation

$$
\partial_{t t} u-\Delta u-f(u)=0
$$

which, since the pioneering [Jörgens 1961], has been the subject of a considerable amount of work. For the energy-critical nonlinearity $f(u)= \pm t^{(N+2) /(N-2)}$, the Cauchy problem is locally well posed in the energy space $\dot{H}^{1} \times L^{2}$ and the solution propagates regularity; see [Sogge 1995] and references therein. Recall that in this case, (1-2) admits a conserved energy

$$
E(u(t))=E\left(u_{0}, u_{1}\right)=\frac{1}{2} \int\left(\partial_{t} u\right)^{2}+\frac{1}{2} \int|\nabla u|^{2} \mp \frac{N-2}{2 N} \int u^{2 N /(N-2)}
$$

that is left invariant by the scaling symmetry of the flow,

$$
u_{\lambda}(t, x)=\frac{1}{\lambda^{(N-2) / 2}} u\left(\frac{t}{\lambda}, \frac{x}{\lambda}\right) .
$$

Global existence in the defocusing case was proved by Struwe [1988] for radial data and Grillakis [1990] for general data. For focusing nonlinearities, a sharp threshold criterion of global existence and scattering

MSC2010: 35Q51.

Keywords: wave equation, blow-up. 
or finite time blow-up is obtained by Kenig and Merle [2008] based on the soliton solution to (1-1),

$$
Q(r)=\left(\frac{1}{1+r^{2} /(N(N-2))}\right)^{(N-2) / 2},
$$

which is the extremizing profile of the Sobolev embedding $\dot{H}^{1} \rightarrow L^{2^{*}}$. Indeed, for initial data $\left(u_{0}, u_{1}\right)$ such that $E\left(u_{0}, u_{1}\right)<E(Q, 0)$, those with $\left\|\nabla u_{0}\right\|_{L^{2}}<\|\nabla Q\|_{L^{2}}$ have global solutions and scatter, while those with $\left\|\nabla u_{0}\right\|_{L^{2}}>\|\nabla Q\|_{L^{2}}$ lead to finite time blow-up.

Note that like in the works of Levine [1974] (see also [Strauss 1989]) and as is standard in a nonlinear dispersive setting, blow-up is derived through obstructive convexity arguments; see also [Karageorgis and Strauss 2007] for refined statements near the soliton $Q$. However, this approach gives very little insight into the description of the blow-up mechanism and the description of the flow even just near the ground state soliton $Q$ is still only at its beginning.

On the energy-critical wave map problem. There is an important literature devoted to the construction of blow-up solutions for nonlinear wave equations; see [Alinhac 1995; Merle and Zaag 2003; 2008] for the study of the ODE-type of blow-up for subcritical nonlinearities. For energy-critical problems like (1-1), recent important progress has been made through the study of the two-dimensional energy-critical corotational wave map to the 2-sphere,

$$
\partial_{t t} u-\partial_{r r} u-\frac{\partial_{r} u}{r}-\frac{k^{2} \sin 2 u}{2 r^{2}}=0,
$$

where $k \in \mathbb{N}^{*}$ is the homotopy number. The ground state is given there by

$$
Q(r)=2 \tan ^{-1}\left(r^{k}\right) .
$$

After the pioneering works of Christodoulou and Tahvildar-Zadeh [1993], Shatah and Tahvildar-Zadeh [1994] and Struwe [2003] and their detailed study of the concentration of energy scenario, the first explicit description of singularity formation for the $k=1$ case was derived by Krieger, Schlag and Tataru [2008] who constructed finite energy finite time blow-up solutions of the form

$$
u(t, x)=(Q+\varepsilon)\left(t, \frac{x}{\lambda(t)}\right), \quad \text { with }\left\|\varepsilon(t), \partial_{t} \varepsilon(t)\right\|_{\dot{H}^{1} \times L^{2}} \ll 1,
$$

with a blow-up speed given by

$$
\lambda(t)=(T-t)^{v}
$$

for any $v>\frac{3}{2}$; see also [Krieger et al. 2009a]. The spectacular feature of this result is that it exhibits arbitrarily slow blow-up regimes further and further from self-similarity which would correspond to the (forbidden; see [Struwe 2003]) self-similar law

$$
\lambda(t) \sim T-t .
$$

Numerics suggest that this blow-up scenario is nongeneric and corresponds to finite-codimensional manifolds [Bizoń et al. 2001]. After the pioneering work [Rodnianski and Sterbenz 2010] for large homotopy number $k \geq 4$, Raphaël and Rodnianski [2012] gave a complete description of stable blow-up 
dynamics that originate from smooth data for all homotopy numbers $k \geq 1$. The blow-up speed obeys in this regime a universal law that depends in an essential way on the rate of convergence of the ground state $Q$ to its asymptotic value,

$$
\pi-Q \sim \frac{1}{r^{k}} \quad \text { as } r \rightarrow \infty,
$$

and indeed the stable blow-up regime corresponds to a decomposition (1-5) with blow-up speed

$$
\lambda(t) \sim \begin{cases}c_{k} \frac{T-t}{|\log (T-t)|^{1 /(2 k-2)}} & \text { for } k \geq 2, \\ (T-t) e^{-\sqrt{|\log (T-t)|}} & \text { for } k=1 .\end{cases}
$$

Note that this work draws an important analogy with another critical problem, the $L^{2}$ critical nonlinear Schrödinger equation, where a similar universality of the stable singularity formation near the ground state was proved in [Merle and Raphael 2003; 2004; 2005a; 2005b; 2006; Raphael 2005].

Statement of the result. For the power nonlinearity energy-critical problem (1-1), there has been recent progress towards the understanding of the flow near the solitary wave $Q$. Krieger and Schlag [2007] constructed in dimension $N=3$ a codimension one manifold of initial data near $Q$ that yield global solutions asymptotically converging to the soliton manifold. The strategy developed by Krieger et al. [2008] for the wave map problem has been adapted in [Krieger et al. 2009b] to show in dimension $N=3$ the existence of finite energy finite time blow-up solutions of the form

$$
u(t, x)=\frac{1}{\lambda^{(N-2) / 2}(t)}(Q+\varepsilon)\left(t, \frac{x}{\lambda(t)}\right), \quad \text { with }\left\|\varepsilon(t), \partial_{t} \varepsilon(t)\right\|_{\dot{H}^{1} \times L^{2}} \ll 1,
$$

and with a blow-up speed given by

$$
\lambda(t)=(T-t)^{v},
$$

for any $v>\frac{3}{2}$. The quantization of the energy at blow-up for small type II blow-up solutions in dimension $N \in\{3,5\}$ is proved in [Duyckaerts et al. 2011;2012] in the radial and nonradial cases. In particular, for radial data, if $T<+\infty$ and

$$
\sup _{t \in[0, T]}\left[|\nabla u(t)|_{L^{2}}^{2}+\left.\partial_{t} u\right|_{L^{2}} ^{2}\right] \leq|\nabla Q|_{L^{2}}^{2}+\alpha^{*}, \quad \alpha^{*} \ll 1,
$$

then there exists a dilation parameter $\lambda(t) \rightarrow 0$ as $t \rightarrow T$ and asymptotic profiles $\left(u^{*}, v^{*}\right) \in H^{1} \times L^{2}$ such that

$$
\left(u(t, x)-\frac{1}{\lambda^{(N-2) / 2}(t)} Q\left(\frac{x}{\lambda(t)}\right), \partial_{t} u(t)\right) \rightarrow\left(u^{*}, v^{*}\right) \quad \text { in } \dot{H}^{1} \times L^{2} \text { as } t \rightarrow T ;
$$

see [Merle and Raphael 2005b] for related classification results for the $L^{2}$ critical (NLS).

These works however leave open the question of the existence of smooth type II blow-up solutions. We claim that such smooth type II blow-up solutions can be constructed in dimension $N=4$ as the formal analogue of the singular dynamics exhibited by Raphaël and Rodnianski [2012] for the wave map problem in the least homotopy number class $k=1$. The following theorem is the main result of this paper: 
Theorem 1.1 (existence of smooth type II blow-up solutions in dimension $N=4$ ). Let $N=4$. Then for all $\alpha^{*}>0$, there exist $\mathscr{C}^{\infty}$ initial data $\left(u_{0}, u_{1}\right)$ with

$$
E\left(u_{0}, u_{1}\right)<E(Q, 0)+\alpha^{*}
$$

such that the corresponding solution to the energy-critical focusing wave equation (1-1) blows up in finite time $T=T\left(u_{0}, u_{1}\right)<+\infty$ in a type II regime according to the following dynamics: there exist $\left(u^{*}, v^{*}\right) \in \dot{H}^{1} \times L^{2}$ such that

$$
\left(u(t, x)-\frac{1}{\lambda^{(N-2) / 2}(t)} Q\left(\frac{x}{\lambda(t)}\right), \partial_{t} u(t)\right) \rightarrow\left(u^{*}, v^{*}\right) \quad \text { in } \dot{H}^{1} \times L^{2} \text { as } t \rightarrow T,
$$

with a blow-up speed given by

$$
\lambda(t)=(T-t) e^{-\sqrt{|\log (T-t)|(1+o(1))}} \text { as } t \rightarrow T .
$$

Comments on the result. 1. On the smoothness of the initial data. An important feature of Theorem 1.1 is to exhibit a new blow-up speed which is valid for $\mathscr{C}^{\infty}$ solutions. Indeed, while the Krieger et al. [2009b] approach provides a continuum of blow-up speeds, the exact regularity of the obtained solutions is not known, which is an unpleasant consequence of their construction scheme. In fact, it is expected that $\mathscr{C}^{\infty}$ initial data should lead to quantized blow-up rates hence breaking the continuum of blow-up speeds (1-8), we refer to [van den Berg et al. 2003] for a related discussion in the context of the energy-critical harmonic heat flow. Hence we expect the blow-up rate (1-10) to correspond to the minimal type II blow-up speed of smooth solutions with small supercritical energy. Such a general lower bound on the blow-up rate in the spirit of the one obtained by Merle and Raphael [2006; Raphael 2005] for the $L^{2}$ critical NLS is an open problem. The construction of excited blow-up solutions with other speeds and $\mathscr{C}^{\infty}$ regularity also remains to be done. This problematic is related to the understanding of the structure of the flow near $Q$, which is still in its infancy.

2. On the codimension one manifold. The proof of Theorem 1.1 involves a detailed description of the set of initial data leading to the type II blow-up with speed (1-10). Indeed, given a small enough parameter $b_{0}>0$ and a suitable deformation $Q_{b_{0}}$ of the soliton with

$$
Q_{b_{0}} \rightarrow Q \quad \text { as } b_{0} \rightarrow 0
$$

in some strong sense, we show that for any smooth and radially symmetric excess of energy

$$
\left\|\eta_{0}, \eta_{1}\right\|_{H^{2} \times H^{1}} \lesssim \frac{b_{0}^{2}}{\left|\log \left(b_{0}\right)\right|}
$$

we can find $d_{+}\left(b_{0}, \eta_{0}, \eta_{1}\right) \in \mathbb{R}$ such that the solution to (1-1) with initial data

$$
u_{0}=Q_{b_{0}}+\eta_{0}+d_{+} \psi, \quad u_{1}=b_{0}\left(\frac{N-2}{2} Q_{b_{0}}+y \cdot \nabla Q_{b_{0}}\right)+\eta_{1},
$$

blows up in finite time in the regime described by Theorem 1.1. Here $\psi$ is the bound state of the linearized operator close to $Q$ and generates the unstable mode, we refer to Definition 3.4 and Proposition 3.5 for precise statements. Hence the set of blow-up solutions we construct lives on a codimension one 
manifold in the radial class in some weak sense. Following [Krieger and Schlag 2007; Krieger and Schlag 2009], the proof that this set is indeed a codimension one manifold relies on proving some Lipschitz regularity of the map $\left(b_{0}, \eta_{0}, \eta_{1}\right) \rightarrow d_{+}\left(b_{0}, \eta_{0}, \eta_{1}\right)$, and in particular some local uniqueness to begin with. The analysis in [Krieger and Schlag 2009] shows that this may be a delicate step in some cases. Our solution is constructed using a soft continuous topological argument of Brouwer-type coupled with suitable monotonicity properties in the spirit of Cote, Marte and Merle [2009]. In other related settings (see [Martel 2005; Raphaël and Szeftel 2011]) this strategy has proved to be quite powerful for eventually achieving strong uniqueness results. This interesting question in our setting will require additional efforts and needs to be addressed separately in detail.

3. Extension to higher dimensions. We focus on the case of dimension $N=4$ for the sake of simplicity. Our main objective is to provide a robust framework to construct $\mathscr{C}^{\infty}$ type II blow-up solutions. However, following the heuristic developed in [Raphaël and Rodnianski 2012], the blow-up speed (1-10) corresponds to the $k=1$ case in (1-7), and we similarly conjecture in dimension $N \geq 5$ the existence of type II finite time blow-up solutions close to $Q$ with blow-up speed

$$
\lambda(t) \sim c_{N} \frac{T-t}{|\log (T-t)|^{1 /(N-4)}} .
$$

Note from (1-3) that the higher the dimension, the fastest the decay of the ground state $Q$, and that this should help avoid some difficulties that occur only in low dimension like in [Raphaël and Rodnianski 2012] for large homotopy number $k \geq 4$. We expect the strategy developed in this paper to carry over to the cases $N=5$ and 6 , but the extension to large dimension will be confronted in particular with the difficulty of the lack of smoothness of the nonlinearity. Let us also insist on the fact that the case $N=4$ is in many ways the more delicate one in terms of the strong coupling of the main part of the solution and the outgoing tail due to the slow decay of $Q$, which results in the somewhat pathological blow-up speed (1-10). This comment becomes even more dramatic in dimension $N=3$, where we expect our analysis to be applicable to the construction of $\mathscr{C}^{\infty}$ type II blow-up solutions, but this seems to require a slightly different approach.

\section{Strategy of the proof of Theorem 1.1 .}

Step 1: Approximate self-similar solution. Let $D, \Lambda$ denote the differential operators in (1-18). Exact self-similar solutions to (1-1) of the form

$$
u(t, x)=\frac{1}{\lambda^{(N-2) / 2}(t)} Q_{b}\left(\frac{x}{\lambda(t)}\right), \quad \text { with } b=-\lambda_{t},
$$

where $Q_{b}$ satisfies the self-similar equation

$$
\Delta Q_{b}-b^{2} D \Lambda Q_{b}+Q_{b}^{3}=0
$$

are known to develop a singularity on the light cone $y=(T-t) / \lambda(t)=1 / b$ leading to an unbounded Dirichlet energy $\left\|\nabla Q_{b}\right\|_{L^{2}}=+\infty$; see [Kavian and Weissler 1990]. We therefore assume $0<b \ll 1$ and 
consider a one term expansion approximation

$$
Q_{b}=Q+b^{2} T_{1},
$$

which injected into (1-11) yields, at the order $b^{2}$,

$$
H T_{1}=-D \Lambda Q .
$$

Here $H$ is the linearized operator close to Q given by

$$
H=-\Delta-\frac{N+2}{N-2} Q^{4 /(N-2)}
$$

The spectral structure of $H$ is well known in connection to the fact that $Q$ is an extremizer of the Sobolev embedding $\dot{H}^{1} \rightarrow L^{2^{*}}$, and in the radial sector $H$ admits one nonpositive eigenvalue with well localized eigenvector $\psi$,

$$
H \psi=-\zeta \psi, \quad \zeta>0
$$

and a resonance at the boundary of the continuum spectrum generated by the scaling invariance of (1-1),

$$
H(\Lambda Q)=0, \quad \Lambda Q(r) \sim \frac{C}{r^{N-2}} \text { as } r \rightarrow+\infty .
$$

In order to solve (1-12), we first remove the leading-order growth in the exact solution $T_{1}=\frac{1}{4}|y|^{2} Q$ which is consequence of the flux computation

$$
(D \Lambda Q, \Lambda Q)=\frac{1}{2} \lim _{y \rightarrow+\infty} y^{4}|\Lambda Q|^{2}>0
$$

due to the slow decay of $Q$ in dimension $N=4$ from (1-3). For this, we solve

$$
H T_{1}=-D \Lambda Q+c_{b} \Lambda Q \mathbf{1}_{y \leq 1 / b}, \quad \text { with } c_{b}=\frac{(D \Lambda Q, \Lambda Q)}{\int_{y \leq 1 / b}|\Lambda Q|^{2}} \sim \frac{1}{2|\log b|} \text { as } b \rightarrow 0 .
$$

The purpose of this construction is to yield after a suitable localization process an $o\left(b^{2}\right)$ approximate solution to the self-similar equation (1-11) whose dominant term near and past the light cone is still given by $Q$ itself in the sense that

$$
b^{2}\left|T_{1}\right| \ll Q \quad \text { for } y \geq 1 / b .
$$

This identifies $Q$ as the leading-order radiation term. ${ }^{1}$

Step 2: Bootstrap estimates. We now roughly consider initial data of the form

$$
u_{0}=Q_{b_{0}}+d_{+} \psi+\eta_{0}, \quad u_{1}=b_{0} \Lambda Q_{b_{0}}+\eta_{1}, \quad \text { with }\left|d_{+}\right|+\left\|\eta_{0}, \eta_{1}\right\|_{H^{2} \times H^{1}} \ll b_{0}^{2},
$$

and introduce a modulated decomposition of the flow

$$
u(t, x)=\frac{1}{\lambda^{(N-2) / 2}(t)}\left(Q_{b(t)}+\varepsilon\right)\left(t, \frac{x}{\lambda(t)}\right), \quad b(t)=-\lambda_{t} .
$$

\footnotetext{
${ }^{1}$ See [Raphaël and Rodnianski 2012] for a further discussion on this issue and the role played by the nonvanishing Pohozaev integration (1-16).
} 
Here we face the major difference between the power nonlinearity wave equation (1-1) and the critical wave map problem (1-4), which is the presence of a negative eigenvalue in the first case (1-14) for the linearized operator $H$ close to $Q$. This induces an instability in the modulation equations for $b, \lambda$ that is absent in the wave map case, leading to stable blow-up dynamics. However, we claim that the ODE-type instability generated by (1-14) is the only instability mechanism.

The situation is conceptually similar to the one studied in [Cote et al. 2009] where multisolitary wave solutions are constructed in the supercritical regime despite the presence of exponentially growing modes for the linearized operator which are absent in the subcritical regime. We adapt a similar scheme of proof that does not rely on a fixed point argument to solve the problem from infinity in time, ${ }^{2}$ but by directly following the flow for any initial data of the form (1-17). This reduces the full problem to a one-dimensional dynamical system for which a clever classical continuity argument yields the existence of $d_{+}\left(b_{0}, \eta_{0}, \eta_{1}\right)$ such that the unstable mode is extinct, see Section 5.

The key is hence to control the flow under the a priori control of the unstable mode, and here we adapt the technology developed in [Raphaël and Rodnianski 2012] which relies on monotonicity properties of the linearized Hamiltonian at the $H^{2}$ level of regularity. However, the analysis in [Raphaël and Rodnianski 2012] heavily relies on the existence of a decomposition of the Hamiltonian,

$$
H=A^{*} A, \quad A=-\partial_{y}+V(y),
$$

which is central to the proof of the main monotonicity property and is lost in our setting. This forces us to revisit the approach in several ways, and to rely in particular on fine algebraic properties of the flow $^{3}$ near $Q$ and coercivity properties of suitable quadratic forms in the spirit of [Martel and Merle 2002; Merle and Raphael 2005a] (see Lemma 4.7) which remarkably turn out to be almost explicit thanks to the formula (1-3). We are eventually able to find $d_{+}\left(b_{0}, \eta_{0}, \eta_{1}\right)$ for which, to leading order,

$$
b_{s} \sim-c_{b} b^{2} \sim-\frac{b^{2}}{2|\log b|}, \quad b=-\lambda_{t}, \quad \frac{d s}{d t}=\frac{1}{\lambda}, \quad\left|d_{+}\right|+\left\|\partial_{y y} \varepsilon\right\|_{L^{2}} \ll b^{2},
$$

and whose reintegration in time yields finite time blow-up in the regime described by Theorem 1.1.

Notation. We define differential operators

$$
\Lambda f=\frac{N-2}{2} f+y \cdot \nabla f \quad\left(\dot{H}^{1} \text { scaling }\right), \quad D f=\frac{N}{2} f+y \cdot \nabla f \quad\left(L^{2} \text { scaling }\right) .
$$

Denoting by

$$
(f, g)=\int f g=\int_{0}^{+\infty} f(r) g(r) r^{N-1} d r
$$

the $L^{2}\left(\mathbb{R}^{N}\right)$ radial inner product, we observe the integration by parts formulas

$$
(D f, g)=-(f, D g) \quad \text { and } \quad(\Lambda f, g)+(\Lambda g, f)=-2(f, g) .
$$

\footnotetext{
${ }^{2}$ After renormalization of the time.

${ }^{3}$ See in particular (4-23), (4-38).
} 
Given $f$ and $\lambda>0$, we shall write

$$
f_{\lambda}(t, r)=\frac{1}{\lambda^{(N-2) / 2}} f\left(t, \frac{r}{\lambda}\right),
$$

and the rescaled space variable will always be denoted by

$$
y=\frac{r}{\lambda} .
$$

We let $\chi$ be a smooth positive radial cut off function, $\chi(r)=1$ for $r \leq 1$ and $\chi(r)=0$ for $r \geq 2$. For a given parameter $B>0$, we let

$$
\chi_{B}(r)=\chi\left(\frac{r}{B}\right)
$$

Given $b>0$, we set

$$
B_{0}=\frac{2}{b}, \quad B_{1}=\frac{|\log b|}{b} .
$$

To clarify the exposition we use the notation $a \lesssim b$ for when there exists a constant $C$ with no relevant dependency on $(a, b)$ such that $a \leq C b$. In particular, we do not allow constants $C$ to depend on the parameter $M$ except in Appendix A.

\section{Computation of the modified self-similar profile}

This section is devoted to the construction of an approximate self-similar solution $Q_{b}$ which describes the dominant part of the blow-up profile inside the backward light cone from the singular point $(0, T)$ and displays a slow decay at infinity which is eventually responsible for the modifications to the blow-up speed with respect to the self-similar law. The key to this construction is the fact that the structure of the linearized operator $H$ close to $Q$ is completely explicit in the radial sector thanks to the explicit formulas at hand for the elements of the kernel.

We introduce the direction

$$
\Phi=D \Lambda Q
$$

which displays the cancellation

$$
|\Phi(y)| \lesssim \frac{1}{1+y^{4}}
$$

and the crucial nondegeneracy which follows from the Pohozaev integration by parts formula,

$$
(\Phi, \Lambda Q)=\lim _{y \rightarrow+\infty}\left(\frac{1}{2} y^{4}|\Lambda Q|^{2}\right)=32>0 .
$$

Proposition 2.1 (approximate self-similar solution). Let $M$ denote a large enough constant. Then there exists $b^{*}(M)>0$ small enough such that for all $0<b<b^{*}(M)$, there exists a smooth radially symmetric profile $T_{1}$ satisfying the orthogonality condition

$$
\left(T_{1}, \chi_{M} \Phi\right)=0
$$

such that

$$
P_{B_{1}}=Q+\chi_{B_{1}} b^{2} T_{1}
$$


is an approximate self-similar solution in the following sense. Let

$$
\Psi_{B_{1}}=-\Delta P_{B_{1}}+b^{2} D \Lambda P_{B_{1}}-f\left(P_{B_{1}}\right),
$$

then for all $k \geq 0,0 \leq y \leq 1 / b^{2}$,

$$
\begin{aligned}
\left|\frac{d^{k} T_{1}}{d y^{k}}(y)\right| & \lesssim \frac{1}{1+y^{k}}\left[\frac{1+|\log (b y)|}{|\log b|} \mathbf{1}_{2 \leq y \leq \frac{B_{0}}{2}}+\frac{1}{b^{2} y^{2}|\log b|} \mathbf{1}_{y \geq \frac{B_{0}}{2}}+\frac{\log (M)+|\log (1+y)|}{1+y^{2}}\right], \\
\left|\frac{d^{k}}{d y^{k}} \frac{\partial P_{B_{1}}}{\partial b}\right| & \lesssim \frac{b \mathbf{1}_{y \leq 2 B_{1}}}{1+y^{k}}\left[\frac{1+|\log (b y)|}{|\log b|} \mathbf{1}_{2 \leq y \leq \frac{B_{0}}{2}}+\frac{1}{b^{2} y^{2}|\log b|} \mathbf{1}_{y \geq \frac{B_{0}}{2}}+\frac{\log (M)+|\log (1+y)|}{1+y^{2}}\right],
\end{aligned}
$$

and, for all $k \geq 0, y \geq 0$,

$$
\begin{aligned}
& \left|\frac{d^{k}}{d y^{k}}\left(\Psi_{B_{1}}-c_{b} b^{2} \chi_{B_{0} / 4} \Lambda Q\right)\right| \\
& \lesssim \frac{b^{4}}{1+y^{k}}\left[\frac{1+|\log (b y)|}{|\log b|} \mathbf{1}_{2 \leq y \leq B_{0} / 2}+\frac{1}{b^{2} y^{2}|\log b|} \mathbf{1}_{2 B_{1} \geq y \geq B_{0} / 2}+\frac{\log (M)+|\log (1+y)|}{1+y^{2}} \mathbf{1}_{y \leq 2 B_{1}}\right] \\
& +\frac{b^{2}}{\left(1+y^{4+k}\right)} \mathbf{1}_{y \geq B_{1} / 2}
\end{aligned}
$$

for some constant

$$
c_{b}=\frac{1}{2|\log b|}\left(1+O\left(\frac{1}{|\log b|}\right)\right) .
$$

Proof.

Step 1: Inversion of $H$. The first Green's function of $H$ is given from scaling invariance by

$$
\Lambda Q(y)=\frac{N-2}{2\left(1+y^{2} /(N(N-2))\right)^{N / 2}}\left(1-\frac{y^{2}}{N(N-2)}\right),
$$

which admits the asymptotics

$$
\forall k \geq 0, \quad \frac{d^{k}(\Lambda Q)}{d y^{k}}(y)= \begin{cases}O(1) & \text { as } y \rightarrow 0, \\ O\left(y^{-(N-2+k)}\right) & \text { as } y \rightarrow \infty\end{cases}
$$

Now let

$$
\Gamma(y)=-\Lambda Q(y) \int_{1}^{y} \frac{d s}{s^{N-1}(\Lambda Q)^{2}(s)}
$$

be another (singular at the origin ${ }^{4}$ ) element of the kernel of $H$, which can be found from the Wronskian relation

$$
\Gamma^{\prime} \Lambda Q-\Gamma(\Lambda Q)^{\prime}=\frac{-1}{y^{N-1}}
$$

From this we easily find the asymptotics of $\Gamma^{(k)}$ for any integer $k$ :

$$
\frac{d^{k} \Gamma}{d y^{k}}(y)= \begin{cases}O\left(y^{-(N-2+k)}\right) & \text { as } y \rightarrow 0 \\ O\left(y^{-k}\right) & \text { as } y \rightarrow \infty\end{cases}
$$

\footnotetext{
${ }^{4}$ Note that $\Gamma$ must be smooth at $y=\sqrt{N(N-2)}$, where $\Lambda Q$ vanishes, because of the radial ODE $H \Gamma=0$.
} 
A smooth solution to $H w=F$ is

$$
w(y)=\Gamma(y) \int_{0}^{y} F(s) \Lambda Q(s) s^{N-1} d s-\Lambda Q(y) \int_{0}^{y} F(s) \Gamma(s) s^{N-1} d s .
$$

We now look for a solution to the self-similar equation in the form $Q+b^{2} T_{1}$. This yields

$$
\begin{aligned}
\Psi_{b} & =-\Delta Q_{b}+b^{2} D \Lambda Q_{b}-f\left(Q_{b}\right) \\
& =b^{2}\left(H T_{1}+D \Lambda Q\right)+b^{4} D \Lambda T_{1}-\left[f\left(Q+b^{2} T_{1}\right)-f(Q)-b^{2} f^{\prime}(Q) T_{1}\right] .
\end{aligned}
$$

Step 2: Computation of $T_{1}$. Thanks to the anomalous decay (2-2), we choose $T_{1}$ to be a solution of

$$
\left\{\begin{aligned}
H T_{1}=F & =-D \Lambda Q+c_{b} \chi_{B_{0} / 4} \Lambda Q \\
\left(T_{1}, \chi_{M} \Phi\right) & =0
\end{aligned}\right.
$$

with $c_{b}$ chosen such that

$$
(F, \Lambda Q)=0
$$

That is, from the Pohozaev integration by parts formula — see (1-21) and (2-3) -

$$
\begin{aligned}
c_{b} & =\frac{(D \Lambda Q, \Lambda Q)}{\left(\chi_{B_{0} / 4} \Lambda Q, \Lambda Q\right)}=\frac{1}{2} \frac{\lim _{y \rightarrow+\infty} y^{4}|\Lambda Q(y)|^{2}}{\int \chi_{B_{0} / 4}|\Lambda Q|^{2}} \\
& =\frac{1}{2|\log b|}\left(1+O\left(\frac{1}{|\log b|}\right)\right) \quad \text { as } b \rightarrow 0 .
\end{aligned}
$$

This yields (2-10). Following (2-14), we first consider

$$
\tilde{T}_{1}(y)=\Gamma(y) \int_{0}^{y} F(s) \Lambda Q(s) s^{3} d s-\Lambda Q(y) \int_{0}^{y} F(s) \Gamma(s) s^{3} d s .
$$

The smoothness of $\tilde{T}_{1}$ at the origin follows from (2-18) together with elliptic regularity from (2-16). We now examine the behavior of $\tilde{T}_{1}$ at large $y$.

We first observe that, from the orthogonality (2-17),

$$
\tilde{T}_{1}(y)=-\left[\Gamma(y) \int_{y}^{+\infty} F(s) \Lambda Q(s) s^{3} d s+\Lambda Q(y) \int_{0}^{y} F(s) \Gamma(s) s^{3} d s\right] .
$$

Hence, from the degeneracy $|D \Lambda Q|=O\left(y^{-4}\right)$, this yields that for $B_{0} / 2 \leq y \leq 1 / b^{2}$,

$$
\begin{aligned}
\left|\tilde{T}_{1}(y)\right| & \lesssim \int_{y}^{+\infty} \frac{s^{3}}{\left(1+s^{4}\right)\left(1+s^{2}\right)} d s+\frac{1}{y^{2}}\left[\int_{0}^{y} \frac{1+s^{3}}{1+s^{4}} d s+\left|c_{b}\right| \int_{0}^{B_{0}} \frac{s^{3}}{1+s^{2}} d s\right] \\
& \lesssim \frac{|\log (1+y)|}{1+y^{2}}+\frac{1}{b^{2} y^{2}|\log b|}
\end{aligned}
$$


Similarly, for $1 \leq y \leq B_{0} / 2$,

$$
\begin{aligned}
\left|\tilde{T}_{1}(y)\right| & =\left|\Gamma(y) \int_{y}^{+\infty} F(s) \Lambda Q(s) s^{3} d s+\Lambda Q(y) \int_{0}^{y} F(s) \Gamma(s) s^{3} d s\right| \\
& \lesssim \int_{y}^{+\infty} \frac{s^{3}}{\left(1+s^{4}\right)\left(1+s^{2}\right)} d s+\left|c_{b}\right| \int_{y}^{B_{0}} \frac{s^{3}}{\left(1+s^{2}\right)^{2}} d s+\frac{1}{1+y^{2}}\left[\int_{0}^{y} \frac{s^{3}}{1+s^{4}} d s+\left|c_{b}\right| \int_{0}^{y} \frac{s^{3}}{1+s^{2}} d s\right] \\
& \lesssim \frac{1+|\log (b y)|}{|\log b|}+\frac{|\log (1+y)|}{1+y^{2}} .
\end{aligned}
$$

We now choose, thanks to (2-3),

$$
T_{1}(y)=\tilde{T}_{1}(y)-c \Lambda Q \quad \text { with } c=\frac{\left(\tilde{T}_{1}, \chi_{M} \Phi\right)}{\left(\chi_{M} \Phi, \Lambda Q\right)},
$$

so that the orthogonality condition (2-4) is fulfilled. We note that the bounds (2-19) and (2-20) ensure that $c$ remains bounded by $\log (M)$ uniformly in $M$ and $b$, provided $b$ is chosen sufficiently small with respect to $M$.

This yields (2-7) for $k=0$; the other cases follow similarly.

Step 3: Estimate on $\Psi_{B_{1}}$ and $\partial_{b} \Psi_{B_{1}}$. We now cut off the slow decaying tail $T_{1}$ according to (2-5) and estimate the corresponding error to self-similarity $\Psi_{B_{1}}$ given by (2-6).

We compute

$$
\begin{aligned}
\Psi_{B_{1}}=b^{2} \chi_{B_{1}}\left(H T_{1}+D \Lambda Q\right)+b^{2}\left[-2 \chi_{B_{1}}^{\prime} T_{1}^{\prime}-T_{1} \Delta \chi_{B_{1}}+\right. & \left.\left(1-\chi_{B_{1}}\right) D \Lambda Q+b^{2} D \Lambda\left(\chi_{B_{1}} T_{1}\right)\right] \\
& -\left[f\left(Q+b^{2} \chi_{B_{1}} T_{1}\right)-f(Q)-\chi_{B_{1}} f^{\prime}(Q) T_{1}\right] .
\end{aligned}
$$

Outside the support of $\chi_{B_{1}}$ we have thus $\Psi_{B_{1}}=b^{2} D \Lambda Q$. On the other hand, in dimension $N=4$, we have the Taylor expansion

$$
f\left(Q+b^{2} \chi_{B_{1}} T_{1}\right)-f(Q)-\chi_{B_{1}} f^{\prime}(Q) T_{1}=b^{4} \chi_{B_{1}}^{2} T_{1}^{2}(y) \int_{0}^{1}(1-\tau)\left(Q(y)+\tau b^{2} \chi_{B_{1}} T_{1}(y)\right) d \tau
$$

We thus estimate from (2-7), (2-15), (2-16) and the degeneracy (2-2) for $y \leq 2 B_{1}$ that

$$
\begin{aligned}
\left|\Psi_{B_{1}}-b^{2} c_{b} \chi_{B_{0} / 4} \Lambda Q\right| \lesssim b^{2} \mathbf{1}_{y \geq B_{1} / 2}( & \left.\frac{T_{1}^{\prime}}{1+y}+\frac{T_{1}}{1+y^{2}}+\frac{1}{1+y^{4}}\right) \\
& +b^{4}\left|D \Lambda\left(\chi_{B_{1}} T_{1}\right)\right|+b^{4}\left|T_{1}^{2}(y)\right| \int_{0}^{1}(1-\tau)\left|Q(y)+\tau b^{2} T_{1}(y)\right| d \tau .
\end{aligned}
$$

(2-7) now yields (2-9) for $k=0$. Further derivatives are estimated similarly thanks to the smoothness of the nonlinearity. We emphasize here that, given $B>0$ large, we have $1 /(1+y) \lesssim 1 / B \lesssim 1 /(1+y)$ on the support of $\chi_{B}^{\prime}$, so that differentiating $\chi_{B}$ acts as multiplication by $1 /(1+y)$. Furthermore, we have $1 / B_{1}=o(b)$ so that we can always dominate $1 /(1+y)$ by $b$ on the support of $\chi_{B_{1}}^{\prime}$.

Finally, we compute $\partial_{b} P_{B_{1}}$ from (2-5). 
To this end, we note that $\partial_{b} c_{b}=O\left(\frac{1}{b|\log (b)|^{2}}\right)$ when $b \rightarrow 0$, so that the source term for $T_{1}$ in (2-16) satisfies

$$
\partial_{b} F=\left[O\left(\frac{1}{b|\log b|}\right) \chi_{B_{0} / 4}+O\left(\frac{1}{b|\log b|}\right) \rho_{B_{0} / 4}\right] \Lambda Q,
$$

where $\rho(z)=z \chi^{\prime}(z) \in \mathscr{C}_{c}^{\infty}(0, \infty)$ and we keep the convention for function dilation. Hence, the same arguments as for $T_{1}$ enable us to show first that $\partial_{b} \tilde{T}_{1}$, and then $\partial_{b} T_{1}$, satisfy the estimates

$$
\left|\frac{d^{k} \partial_{b} T_{1}}{d y^{k}}(y)\right| \lesssim \frac{1}{b\left(1+y^{k}\right)}\left[\frac{1+|\log (b y)|}{|\log b|} \mathbf{1}_{2 \leq y \leq B_{0} / 2}+\frac{1}{b^{2} y^{2}|\log b|} \mathbf{1}_{y \geq B_{0} / 2}+\frac{1+|\log (1+y)|}{1+y^{2}}\right] .
$$

Finally, we compute from (2-5) that

$$
\partial_{b} P_{B_{1}}=2 b \chi_{B_{1}} T_{1}+b^{2} \partial_{b} \log \left(B_{1}\right) \rho_{B_{1}} T_{1}+b^{2} \chi_{B_{1}} \partial_{b} T_{1} .
$$

This decomposition, together with (2-7) and the previous computation, yield (2-8), which concludes the proof of Proposition 2.1.

\section{Description of the trapped regime}

We display in this section the regime which leads to the blow-up dynamics described by Theorem 1.1.

Modulation of solutions to (1-1). Let us start by describing the set of solutions among which the finite time blow-up scenario described by Theorem 1.1 is likely to arise. We recall from (1-14) that $\psi$ denotes the bound state of $H$ with eigenvalue $-\zeta<0$. The following lemma is a standard consequence of the implicit function theorem and the smoothness of the flow; see Appendix A.

Lemma 3.1 (modulation theory). Let $M$ be a large constant to be chosen later and $0<b_{0}<b_{0}^{*}(M)$ small enough. Let $\left(\eta_{0}, \eta_{1}, d_{+}\right)$satisfy the smallness condition

$$
\left|d_{+}\right|+\left\|\eta_{0}, \nabla \eta_{0}, \eta_{1}+b_{0}\left(1-\chi_{B_{1}\left(b_{0}\right)}\right) \Lambda Q, \nabla \eta_{1}\right\|_{\dot{H}^{1} \times \dot{H}^{1} \times L^{2} \times L^{2}} \lesssim \frac{b_{0}^{2}}{\left|\log b_{0}\right|},
$$

then there exists a time $T_{0}$ such that the unique solution $u \in \mathscr{b}^{2}\left(\left[0, T_{0}\right] ; L^{2}\left(\mathbb{R}^{N}\right)\right) \cap \mathscr{C}\left(\left[0, T_{0}\right] ; H^{2}\left(\mathbb{R}^{N}\right)\right)$ to (1-1) with initial data

$$
u_{0}=P_{B_{1}\left(b_{0}\right)}+\eta_{0}+d_{+} \psi, \quad u_{1}=b_{0} \Lambda P_{B_{1}\left(b_{0}\right)}+\eta_{1},
$$

admits on $\left[0, T_{0}\right]$ a unique decomposition

$$
u(t)=\left(P_{B_{1}(b(t))}+\varepsilon(t)\right)_{\lambda(t)}
$$

with $\lambda \in \mathscr{C}^{2}\left(\left[0, T_{0}\right], \mathbb{R}_{+}^{*}\right)$ such that

$$
\left(\varepsilon(t), \chi_{M} \Phi\right)=0 \text { and } b(t)=-\lambda_{t} \quad \text { for all } t \in\left[0, T_{0}\right],
$$

and the following smallness condition is satisfied:

$\|\nabla \varepsilon(t)\|_{L^{2}} \lesssim b_{0}\left|\log b_{0}\right|, \quad\left|b(t)-b_{0}\right|+|\lambda(t)-1|+\left\|\nabla^{2} \varepsilon(t)\right\|_{L^{2}} \lesssim \frac{b_{0}^{2}}{\left|\log b_{0}\right|} \quad$ for all $t \in\left[0, T_{0}\right]$ 
Remark 3.2. Recall that the slow decay of $Q$ and the choice of $P_{B_{1}}$ induces an unbounded tail of $\Lambda P_{B_{1}}$ in the energy norm, and more specifically $\|\Lambda Q\|_{L^{2}}=+\infty$, hence the need for the compensation in the norm of the time derivative in (3-1).

Decomposition of the flow and modulation equations. Considering initial data satisfying the assumption of the above lemma, we now write the evolution equation induced by (1-1) in terms of the decomposition (3-3). Let

$$
u(t, r)=\frac{1}{[\lambda(t)]^{N / 2-1}}\left(P_{B_{1}(b(t))}+\varepsilon\right)\left(t, \frac{r}{\lambda(t)}\right)=\left(P_{B_{1}(b(t))}\right)_{\lambda(t)}+w(t, r),
$$

where $b=-\lambda_{t}$. Let us derive the equations for $w$ and $\varepsilon$. Let

$$
s(t)=\int_{0}^{t} \frac{d \tau}{\lambda(\tau)}
$$

be the rescaled time. We shall make an intensive use of the rescaling formulas

$$
\begin{aligned}
u(t, r) & =\frac{1}{\lambda^{N / 2}-1} v(s, y), \quad y=\frac{r}{\lambda}, \quad \frac{d s}{d t}=\frac{1}{\lambda}, \\
\partial_{t} u & =\frac{1}{\lambda}\left(\partial_{s} v+b \Lambda v\right)_{\lambda}, \\
\partial_{t t} u & =\frac{1}{\lambda^{2}}\left[\partial_{s}^{2} v+b\left(\partial_{s} v+2 \Lambda \partial_{s} v\right)+b^{2} D \Lambda v+b_{s} \Lambda v\right]_{\lambda} .
\end{aligned}
$$

In particular, we derive from (1-1) the equation for $\varepsilon$,

$\partial_{s}^{2} \varepsilon+H_{B_{1}} \varepsilon=-\Psi_{B_{1}}-b_{s} \Lambda P_{B_{1}}-b\left(\partial_{s} P_{B_{1}}+2 \Lambda \partial_{s} P_{B_{1}}\right)-\partial_{s}^{2} P_{B_{1}}-b\left(\partial_{s} \varepsilon+2 \Lambda \partial_{s} \varepsilon\right)-b_{s} \Lambda \varepsilon+N(\varepsilon)$,

where, implicitly, $B_{1}=B_{1}(b(t))$ and $H_{B_{1}}$ is the linear operator associated to the profile $P_{B_{1}}$,

$$
H_{B_{1}} \varepsilon=-\Delta \varepsilon+b^{2} D \Lambda \varepsilon-f^{\prime}\left(P_{B_{1}}\right) \varepsilon,
$$

and the nonlinearity

$$
N(\varepsilon)=f\left(P_{B_{1}}+\varepsilon\right)-f\left(P_{B_{1}}\right)-f^{\prime}\left(P_{B_{1}}\right) \varepsilon .
$$

Alternatively, the equation for $w$ takes the form

$$
\partial_{t}^{2} w+\tilde{H}_{B_{1}} w=-\left[\partial_{t}^{2}\left(P_{B_{1}}\right)_{\lambda}-\Delta\left(P_{B_{1}}\right)_{\lambda}-f\left(\left(P_{B_{1}}\right)_{\lambda}\right)\right]+N_{\lambda}(w),
$$

with

$$
\begin{aligned}
\tilde{H}_{B_{1}} w & =-\Delta w-f^{\prime}\left(\left(P_{B_{1}}\right)_{\lambda}\right) w \\
N_{\lambda}(w) & =f\left(\left(P_{B_{1}}\right)_{\lambda}+w\right)-f\left(\left(P_{B_{1}}\right)_{\lambda}\right)-f^{\prime}\left(\left(P_{B_{1}}\right)_{\lambda}\right) w .
\end{aligned}
$$


We then expand using (3-9), (3-10), obtaining

$$
\begin{aligned}
\partial_{t}^{2}\left(P_{B_{1}}\right)_{\lambda}-\Delta\left(P_{B_{1}}\right)_{\lambda}-f\left(\left(P_{B_{1}}\right)_{\lambda}\right) & =\frac{1}{\lambda^{2}}\left[\partial_{s s} P_{B_{1}}+b\left(\partial_{s} P_{B_{1}}+2 \Lambda \partial_{s} P_{B_{1}}\right)+b_{s} \Lambda P_{B_{1}}+\Psi_{B_{1}}\right]_{\lambda} \\
& =\frac{1}{\lambda^{2}}\left[b \Lambda \partial_{s} P_{B_{1}}+b_{s} \Lambda P_{B_{1}}+\Psi_{B_{1}}\right]_{\lambda}+\partial_{t}\left[\frac{1}{\lambda}\left(\partial_{s} P_{B_{1}}\right)_{\lambda}\right],
\end{aligned}
$$

and rewrite the equation for $w$ as

$$
\partial_{t}^{2} w+\tilde{H}_{B_{1}} w=-\frac{1}{\lambda^{2}}\left[b \Lambda \partial_{s} P_{B_{1}}+b_{s} \Lambda P_{B_{1}}+\Psi_{B_{1}}\right]_{\lambda}-\partial_{t}\left[\frac{1}{\lambda}\left(\partial_{s} P_{B_{1}}\right)_{\lambda}\right]+N_{\lambda}(w) .
$$

For most of our arguments we prefer to view the linear operator $H_{B_{1}}$ acting on $w$ in (3-16) as a perturbation of the linear operator $H_{\lambda}$ associated to $Q_{\lambda}$. Then

$$
\begin{aligned}
\partial_{t}^{2} w+H_{\lambda} w & =F_{B_{1}} \\
& =-\frac{1}{\lambda^{2}}\left[b \Lambda \partial_{s} P_{B_{1}}+b_{s} \Lambda P_{B_{1}}+\Psi_{B_{1}}\right]_{\lambda}-\partial_{t}\left[\frac{1}{\lambda}\left(\partial_{s} P_{B_{1}}\right)_{\lambda}\right]-\left[f^{\prime}\left(Q_{\lambda}\right)-f^{\prime}\left(\left(P_{B_{1}}\right)_{\lambda}\right)\right] w+N_{\lambda}(w)
\end{aligned}
$$

with

$$
H_{\lambda} w=-\Delta w+f^{\prime}\left(Q_{\lambda}\right) w
$$

The set of bootstrap estimates. First we fix some notations. We introduce the energy $\mathscr{E}(t)$ associated to the Hamiltonian $H_{\lambda}$,

$$
\mathscr{E}(t)=\lambda^{2} \int\left[\left(H_{\lambda} \partial_{t} w, \partial_{t} w\right)+\left(H_{\lambda} w\right)^{2}\right]
$$

Given the unstable eigenvalue $\zeta \in(0, \infty)$, we set

$$
V_{+}=\left|\begin{array}{c}
1 \\
\sqrt{\zeta}
\end{array}, \quad V_{-}=\right| \begin{gathered}
1 \\
-\sqrt{\zeta}
\end{gathered}
$$

and introduce the decomposition of the unstable direction,

$$
\mid \begin{aligned}
& (\varepsilon, \psi) \\
& \left(\partial_{s} \varepsilon, \psi\right)
\end{aligned}=\tilde{a}_{+}(s) V_{+}+\tilde{a}_{-}(s) V_{-} .
$$

Let us write

$$
\kappa_{+}(s)=\tilde{a}_{+}(s)+\frac{b_{s}}{2 \sqrt{\zeta}}\left(\partial_{b} P_{B_{1}}, \psi\right), \quad \kappa_{-}(s)=\tilde{a}_{-}(s)-\frac{b_{s}}{2 \sqrt{\zeta}}\left(\partial_{b} P_{B_{1}}, \psi\right) .
$$

We note that the vectors $V_{+}, V_{-}$given by (3-20) yield an eigenbasis of

$$
\left(\begin{array}{ll}
0 & 1 \\
\zeta & 0
\end{array}\right)
$$

and hence correspond respectively to the unstable and stable mode of the two dimensional dynamical system

$$
\frac{d Y}{d s}=\left(\begin{array}{ll}
0 & 1 \\
\zeta & 0
\end{array}\right) Y
$$


which, to first order in $b$, is verified by the projection onto the unstable mode $(\varepsilon, \psi)$; see (4-57). The deformation term $b_{s}\left(\partial_{b} P_{B_{1}}, \psi\right)$ in (3-22) is present to handle some possible time oscillations induced by the $\partial_{s}^{2} P_{B_{1}}$ term in the right-hand side of (3-11), which cannot be estimated in absolute value but will be proved to be of lower order.

With these conventions, we may now parametrize the set of initial data described by Lemma 3.1 by $a_{+}=\kappa_{+}(0)$, and then reformulate the initial smallness properties in terms of suitable initial bounds for $\varepsilon$; see Appendix A for the proof, which is standard.

Lemma 3.3 (initial parametrization of the unstable mode and initial bounds). Let $M$ and $b_{0}$ be given as in Lemma 3.1 and denote by $C(M)$ a sufficiently large constant. Then, given $\left(\eta_{0}, \eta_{1}, a_{+}\right)$satisfying

$$
\left|a_{+}\right|+\left\|\eta_{0}, \nabla \eta_{0}, \eta_{1}+b_{0}\left(1-\chi_{B_{1}\left(b_{0}\right)}\right) \Lambda Q, \nabla \eta_{1}\right\|_{\dot{H}^{1} \times \dot{H}^{1} \times L^{2} \times L^{2}} \leq \frac{b_{0}^{2}}{\left|\log b_{0}\right|},
$$

there exists a unique $d_{+}$with $\left|d_{+}\right| \lesssim b_{0}^{2} /\left|\log \left(b_{0}\right)\right|$ and $T_{0}>0$ such that the unique decomposition

$$
u(t)=\left(P_{B_{1}(b(t))}+\varepsilon\right)_{\lambda(t)}=\left(P_{B_{1}(b(t))}\right)_{\lambda(t)}+w(t)
$$

of the unique smooth solution u to (1-1) on $\left[0, T_{0}\right]$ with initial data (3-2) satisfies the initialization

$$
\kappa_{+}(0)=a_{+}
$$

and the following smallness conditions on $\left[0, T_{0}\right]$ :

- Smallness and positivity of $b$ :

$$
0<b(t)<5 b_{0}
$$

- Pointwise bound on $b_{s}$ :

$$
\left|b_{s}(t)\right|^{2} \leq C(M) \frac{[b(t)]^{4}}{|\log b(t)|^{2}} .
$$

- Smallness of the energy norm:

$$
\left\|\nabla w(t), \partial_{t} w(t)+\frac{b(t)}{\lambda(t)}\left(\left(1-\chi_{B_{1}(b(t))}\right) \Lambda Q\right)_{\lambda(t)}\right\|_{L^{2} \times L^{2}} \leq \sqrt{b_{0}} .
$$

- Global $\dot{H}^{2}$ bound:

$$
|\mathscr{E}(t)| \leq C(M) \frac{[b(t)]^{4}}{|\log b(t)|^{2}} .
$$

- A priori bound on the stable mode:

$$
\left|\kappa_{-}(t)\right| \leq(C(M))^{1 / 8} \frac{[b(t)]^{2}}{|\log b(t)|} .
$$

- A priori bound of the unstable mode:

$$
\left|\kappa_{+}(t)\right| \leq 2 \frac{[b(t)]^{2}}{|\log b(t)|} .
$$

We can now describe the bootstrap regime. 
Definition 3.4 (exit time). Let $K(M)$ be a large constant. Given $a_{+} \in\left[-b_{0}^{2} /\left|\log b_{0}\right|, b_{0}^{2} /\left|\log b_{0}\right|\right]$, we let $T\left(a_{+}\right)$be the life time of the solution to (1-1) with initial data (3-2), and $T_{1}\left(a_{+}\right)>0$ be the supremum of $T \in\left(0, T\left(a_{+}\right)\right)$such that for all $t \in[0, T]$, the following estimates hold:

- Smallness and positivity of $b$ :

$$
0<b(t)<5 b_{0}
$$

- Pointwise bound on $b_{s}$ :

$$
\left|b_{s}\right|^{2} \leq K(M) \frac{[b(t)]^{4}}{|\log b(t)|^{2}}
$$

- Smallness of the energy norm:

$$
\left\|\nabla w(t), \partial_{t} w(t)+\frac{b(t)}{\lambda(t)}\left(\left(1-\chi_{B_{1}(b(t))}\right) \Lambda Q\right)_{\lambda(t)}\right\|_{L^{2} \times L^{2}} \leq \sqrt{b_{0}} .
$$

- Global $\dot{H}^{2}$ bound:

$$
|\mathscr{E}(t)| \leq K(M) \frac{[b(t)]^{4}}{|\log b(t)|^{2}} .
$$

- A priori bound on the stable and unstable modes:

$$
\left|\kappa_{+}(t)\right| \leq 2 \frac{[b(t)]^{2}}{|\log b(t)|}, \quad\left|\kappa_{-}(t)\right| \leq(K(M))^{1 / 8} \frac{[b(t)]^{2}}{|\log b(t)|} .
$$

The existence of blow-up solutions in the regime described by Theorem 1.1 now follows from the following proposition:

Proposition 3.5. There exists $a_{+} \in\left[-b_{0}^{2} /\left|\log b_{0}\right|, b_{0}^{2} /\left|\log b_{0}\right|\right]$ such that

$$
T_{1}\left(a_{+}\right)=T\left(a_{+}\right) .
$$

Then the corresponding solution to (1-1) blows up in finite time in the regime described by Theorem 1.1.

The proof of Proposition 3.5 relies on a monotonicity argument applied to the energy $\mathscr{E}$, which is the core of the analysis (see Proposition 4.6), and the strictly outgoing behavior of the unstable mode induced by the nontrivial eigenvalue $-\zeta<0$ of $H$ (see Lemma 4.10). The fact that the regime described by the bootstrap bounds (3-31)-(3-35) corresponds to a finite blow-up solution with a specific blow-up speed will then follow from the modulation equations and the sharp derivation of the blow speed as in [Raphaël and Rodnianski 2012].

\section{Improved bounds}

This section is devoted to the derivation of the main dynamical properties of the flow in the bootstrap regime described by Definition 3.4. The three main steps are first the derivation of a monotonicity property on $\mathscr{E}$, which allows us to improve the bounds (3-31)-(3-34) in $\left[0, T_{1}\left(a_{+}\right)\right]$, second the derivation of the dynamics of the eigenmode and the outgoing behavior of the unstable direction, and third the derivation 
of the sharp law for the parameter $b$, which allows to bootstrap its smallness (3-31) and will eventually allow us to derive the sharp blow-up speed.

Remark 4.1. Throughout the proof, we will introduce various constants $C(M), \delta(M)>0$ that do not depend on the bootstrap constant $K(M)$. An important feature of all these constants is that, up to a smaller choice of $b^{*}(M)$ or a larger choice of $K(M)$, we assume that any product of the form $C(M) f(b)$, where $\lim _{b \rightarrow 0} f(b)=0$, or that any ratio $\delta(M) / K(M)$ is small in the trapped regime. This will be used implicitly in this section.

Coercivity of $\mathscr{E}$. Let us start by showing that the linearized energy $\mathscr{E}$ yields a control of suitable weighted norms of $(w, \varepsilon)$ in the regime $t \in\left[0, T_{1}\left(a_{+}\right)\right]$.

Lemma 4.2 (coercivity of $\mathscr{E}$ ). There exists $M_{0} \geq 1$ such that for all $M \geq M_{0}$, there exists ${ }^{5} \delta(M)>0$ and $C(M)<\infty$ such that in the interval $\left[0, T_{1}\left(a_{+}\right)\right)$,

$$
\mathscr{E} \geq \frac{1}{2} \lambda^{2} \int\left(H_{\lambda} w\right)^{2}+\delta(M) \lambda^{2}\left[\int\left(\nabla \partial_{t} w\right)^{2}+\int \frac{\left(\partial_{r} w\right)^{2}}{r^{2}}\right]-C(M)[K(M)]^{1 / 4} \frac{b^{4}}{|\log b|^{2}} .
$$

Proof of Lemma 4.2. This is a consequence of the explicit distribution of the negative eigenvalues of $H$ and the a priori bound on the unstable mode (3-35). Indeed, let $t \in\left[0, T_{1}\left(a_{+}\right)\right.$), then first observe from (3-21), (3-22), (3-35) that

$$
\begin{aligned}
|(\varepsilon, \psi)|^{2}+\left|\left(\partial_{s} \varepsilon, \psi\right)\right|^{2} & \lesssim\left|\kappa_{+}\right|^{2}+\left|\kappa_{-}\right|^{2}+\left|b_{s}\right|^{2}\left(\partial_{b} P_{B_{1}}, \psi\right)^{2} \\
& \lesssim[K(M)]^{1 / 4} \frac{b^{4}}{|\log b|^{2}}+C(M) b^{2}\left|b_{s}\right|^{2} \lesssim[K(M)]^{1 / 4} b^{4} /|\log b|^{2},
\end{aligned}
$$

where we used the estimates of Proposition 2.1 and the fact that $\psi$ is well localized. This yields

$$
\begin{aligned}
\frac{1}{\lambda^{4}}\left(w, \psi_{\lambda}\right)^{2}+\frac{1}{\lambda^{2}}\left(\partial_{t} w, \psi_{\lambda}\right)^{2} & =(\varepsilon, \psi)^{2}+\left(\partial_{s} \varepsilon+b \Lambda \varepsilon, \psi\right)^{2} \\
& \lesssim[K(M)]^{1 / 4} \frac{b^{4}}{|\log b|^{2}}+b^{2}\left[\int \frac{\varepsilon^{2}}{y^{4}(1+|\log (y)|)^{2}}+\int \frac{|\nabla \varepsilon|^{2}}{y^{2}}\right],
\end{aligned}
$$

and similarly, using the orthogonality condition (3-4),

$$
\begin{aligned}
\frac{1}{\lambda^{4}}\left(w,\left(\chi_{M} \Phi\right)_{\lambda}\right)^{2}+\frac{1}{\lambda^{2}}\left(\partial_{t} w,\left(\chi_{M} \Phi\right)_{\lambda}\right)^{2} & =\left(b \Lambda \varepsilon, \chi_{M} \Phi\right)^{2} \\
& \lesssim b^{2} M^{C}\left[\int \frac{\varepsilon^{2}}{y^{4}(1+|\log (y)|)^{2}}+\int \frac{|\nabla \varepsilon|^{2}}{y^{2}}\right] .
\end{aligned}
$$

Applying Lemma C.3 yields

$$
\lambda^{2} \int\left|H_{\lambda} w\right|^{2}=\int|H \varepsilon|^{2} \geq \delta(M)\left[\int \frac{|\nabla \varepsilon|^{2}}{y^{2}}+\frac{\varepsilon^{2}}{y^{4}(1+|\log (y)|)^{2}}\right] .
$$

\footnotetext{
${ }^{5}$ Recall Remark 4.1.
} 
Introducing the rescaled version $(\mathrm{C}-13)$ of Lemma C.3, we then conclude that

$$
\begin{aligned}
\mathscr{E} \geq \frac{1}{2} \int \lambda^{2}\left(H_{\lambda} w\right)^{2}+\delta_{1}(M)[ & \left.\lambda^{2} \int\left(\nabla \partial_{t} w\right)^{2}+\int \frac{|\nabla \varepsilon|^{2}}{y^{2}}+\int \frac{\varepsilon^{2}}{y^{4}(1+|\log (y)|)^{2}}\right] \\
& -b^{2} M^{C}\left[\int \frac{\varepsilon^{2}}{y^{4}(1+|\log (y)|)^{2}}+\int \frac{|\nabla \varepsilon|^{2}}{y^{2}}\right]-C(M)[K(M)]^{1 / 4} \frac{b^{4}}{|\log b|^{2}} \\
\geq \frac{1}{2} \int \lambda^{2}\left(H_{\lambda} w\right)^{2}+\delta(M) \lambda^{2} & {\left[\int\left(\nabla \partial_{t} w\right)^{2}+\int \frac{\left(\partial_{r} w\right)^{2}}{r^{2}}\right]-C(M)[K(M)]^{1 / 4} \frac{b^{4}}{|\log b|^{2}} }
\end{aligned}
$$

where we used the Hardy bound (C-3), and (4-1) is proved.

Remark 4.3. Note that (4-1) together with the Hardy estimate (C-1), the coercivity estimate (C-9) and (4-4) yield the following weighted bound on $\varepsilon$ which will be extensively used in the paper: Let

$$
\eta(s, y)=\lambda^{(N-2) / 2+1} \partial_{t} w(t, \lambda y)=\partial_{s} \varepsilon(s, y)+b \Lambda \varepsilon(s, y)
$$

then

$$
\begin{aligned}
\int \frac{\varepsilon^{2}}{y^{4}\left(1+|\log y|^{2}\right)}+\int \frac{\eta^{2}}{y^{2}}+\int \frac{|\nabla \varepsilon|^{2}}{y^{2}}+\int|\nabla \eta|^{2} & \lesssim c(M)\left[|\mathscr{E}|+[K(M)]^{1 / 4} \frac{b^{4}}{|\log b|^{2}}\right] \\
& \lesssim c(M)|\mathscr{E}|+\sqrt{K(M)} \frac{b^{4}}{|\log b|^{2}}
\end{aligned}
$$

First bound on $\boldsymbol{b}_{\boldsymbol{s}}$. We now derive a crude bound on $b_{s}$ which appears as an order-one forcing term in the right-hand side of the equation (3-11) for $\varepsilon$. This bound is a simple consequence of the construction of the profile $Q_{b}$ and the choice of the orthogonality condition (3-4).

Lemma 4.4 (rough pointwise bound on $b_{s}$ ). We have the bound 6

$$
\left(b_{s}+\frac{(\varepsilon, H \Phi)}{(\Lambda Q, \Phi)}\right)^{2} \lesssim \frac{1}{M}|\mathscr{E}|+\sqrt{K(M)} \frac{b^{4}}{|\log b|^{2}} .
$$

Remark 4.5. This is in contrast with [Raphaël and Rodnianski 2012], where the $b_{s}$ term could be treated as degenerate with respect to $\varepsilon$ thanks to a specific choice of orthogonality conditions and the factorization of the operator $H$ in the wave map case. This difficulty in our case will be treated using a specific algebra generated by our choice of orthogonality condition (3-4) which gives the right sign to the leading-order terms involving $b_{s}$ in the energy identity of Proposition 4.6; see (4-24), (4-38).

Proof of Lemma 4.4. Let us recall that the equation for $\varepsilon$ in rescaled variables is given by (3-11)-(3-13). Observe also that from (1-19), the adjoint of $H_{B}$ with respect to the $L^{2}\left(\mathbb{R}^{N}\right)$ inner product is

$$
H_{B_{1}}^{*}=H_{B_{1}}+2 b^{2} D \text {. }
$$

To compute $b_{s}$ we take the scalar product of (3-11) with $\chi_{M} \Phi$. Using the orthogonality relations

$$
\left(\partial_{s}^{m} \varepsilon, \chi_{M} \Phi\right)=\left(\partial_{s}^{m}\left(P_{B_{1}}-Q\right), \chi_{M} \Phi\right)=0 \text { for all } m \geq 0
$$

\footnotetext{
${ }^{6}$ Recall Remark 4.1.
} 
we integrate by parts to get the algebraic identity

$$
\begin{aligned}
& b_{s}\left[\left(\Lambda P_{B_{1}}, \chi_{M} \Phi\right)+2\right.\left.b\left(\Lambda \partial_{b} P_{B_{1}}, \chi_{M} \Phi\right)+\left(\Lambda \varepsilon, \chi_{M} \Phi\right)\right] \\
&=-\left(\Psi_{B_{1}}, \chi_{M} \Phi\right)-\left(\varepsilon, H_{B_{1}}^{*}\left(\chi_{M} \Phi\right)\right)+2 b\left(\partial_{s} \varepsilon, \Lambda\left(\chi_{M} \Phi\right)\right)+\left(N(\varepsilon), \chi_{M} \Phi\right) .
\end{aligned}
$$

We first derive from the estimates of Proposition 2.1 that

$$
\left(\Psi_{B_{1}}, \chi_{M} \Phi\right)^{2} \lesssim \frac{b^{4}}{|\log b|^{2}}
$$

Similarly, using (4-6) yields

$$
\left(\partial_{s} \varepsilon, \Lambda\left(\chi_{M} \Phi\right)\right)^{2} \lesssim C(M)\left[c(M)|\mathscr{E}|+\sqrt{K(M)} \frac{b^{4}}{|\log b|^{2}}\right]
$$

and

$$
\left(\varepsilon, H_{B_{1}}^{*}\left(\chi_{M} \Phi\right)\right)=(\varepsilon, H \Phi)-\left(H \varepsilon,\left(1-\chi_{M}\right) \Phi\right)+O\left(M^{C} b^{2} \sqrt{c(M)|\mathscr{E}|+\sqrt{K(M)} b^{4} /|\log b|^{2}}\right) .
$$

We then use the improved decay (2-2) and (4-7) to estimate

$$
\left(H \varepsilon,\left(1-\chi_{M}\right) \Phi\right)^{2} \lesssim\left(\int_{y \geq M} \frac{|H \varepsilon|}{1+y^{N}}\right)^{2} \lesssim \frac{|\mathscr{E}|}{M}+\sqrt{K(M)} \frac{b^{4}}{|\log b|^{2}}
$$

Thus

$$
\left|\left(\varepsilon, H_{B_{1}}^{*}\left(\chi_{M} \Phi\right)\right)-(\varepsilon, H \Phi)\right|^{2} \lesssim \frac{1}{M}|\mathscr{E}|+\sqrt{K(M)} \frac{b^{4}}{|\log b|^{2}}
$$

Similarly,

$$
\begin{aligned}
\left(\Lambda P_{B_{1}}, \chi_{M} \Phi\right)+2 b\left(\Lambda \partial_{b} P_{B_{1}}, \chi_{M} \Phi\right) & +\left(\Lambda \varepsilon, \chi_{M} \Phi\right) \\
= & (\Lambda Q, \Phi)+O\left(\frac{b}{|\log b|}+M^{C} \sqrt{|\mathscr{E}|+\sqrt{K(M)} b^{4} /|\log b|^{2}}\right) \\
= & (\Lambda Q, \Phi)+O\left(\frac{b}{|\log b|}\right)
\end{aligned}
$$

where we have used that in the trapped regime we have $\mathscr{E} \leq K(M) b^{4} /[\log (b)]^{2}$. Finally, on the support of $\chi_{M}$ and for $b<b_{0}^{*}(M)$ small enough, the term $Q$ dominates in $Q_{b}=Q+b^{2} T_{1}$. Hence, for the nonlinear term, we have from the Sobolev inequality and (4-7) that

$$
\left|\left(N(\varepsilon), \chi_{M} \Phi\right)\right| \lesssim \int\left(\frac{\varepsilon^{2}}{1+y^{6}}+\frac{\varepsilon^{3}}{1+y^{4}}\right) \lesssim \int \frac{|\varepsilon|^{2}}{\left(1+y^{5}\right)}\left[1+\|y \varepsilon\|_{L^{\infty}}\right] \lesssim C(M)\left[\mathscr{E}+\sqrt{K(M)} \frac{b^{4}}{|\log b|^{2}}\right] .
$$

Injecting this, together with (4-11)-(4-14), into (4-10) yields (4-8). ${ }^{7}$

\footnotetext{
${ }^{7}$ Recall Remark 4.1 .
} 
Global $\dot{\boldsymbol{H}}^{2}$ bound. We derive in this section a monotonicity statement for the energy $\mathscr{E}$ that provides a global $\dot{H}^{2}$ estimate for the solution. The monotonicity statement involves suitable repulsive properties of the rescaled Hamiltonian $H_{\lambda}$ in the focusing regime under the orthogonality condition (3-11) and the $a$ priori control of the unstable mode (3-35), which themselves rely on the positivity of an explicit quadratic form; see Lemma 4.7.

Proposition 4.6 ( $H^{2}$ control of the radiation). In the trapped regime, there exists a function $\mathscr{F}$ satisfying

$$
\mathscr{F} \lesssim \frac{\mathscr{E}}{M}+\sqrt{K(M)} \frac{b^{4}}{|\log b|^{2}}
$$

such that, for some $0<\alpha<1$ close enough to 1 , we have

$$
\frac{d}{d t}\left\{\frac{\mathscr{E}+\mathscr{F}}{\lambda^{2(1-\alpha)}}\right\} \leq \frac{b}{\lambda^{3-2 \alpha}}\left[\sqrt{K(M)} \frac{b^{4}}{|\log b|^{2}}\right]
$$

Proof.

Step 1: Energy identity. Let

$$
\widetilde{V}(t, r)=\frac{N+2}{N-2} Q_{\lambda}^{4 /(N-2)}(r)=\frac{1}{\lambda^{2}} V\left(\frac{r}{\lambda}\right), \quad V(y)=\frac{N+2}{N-2} Q^{4 /(N-2)}(y) .
$$

We first have an algebraic energy identity that follows by integrating by parts from (3-17),

$$
\frac{1}{2} \frac{d}{d t}\left\{\int\left(\partial_{t r} w\right)^{2}-\int \widetilde{V}\left(\partial_{t} w\right)^{2}+\int\left(H_{\lambda} w\right)^{2}\right\}=-\int \partial_{t} \widetilde{V}\left[\frac{\left(\partial_{t} w\right)^{2}}{2}+w H_{\lambda} w\right]+\int \partial_{t} w H_{\lambda} F_{B_{1}} .
$$

We now use the $w$ equation and integration by parts to compute

$$
\begin{aligned}
-\int \partial_{t} \widetilde{V} w H_{\lambda} w & =-\int \partial_{t} \widetilde{V} w\left(F_{B_{1}}-\partial_{t t} w\right) \\
& =\frac{d}{d t}\left\{\int \partial_{t} \widetilde{V} w \partial_{t} w\right\}-\int \partial_{t} \widetilde{V} w F_{B_{1}}-\int \partial_{t} \widetilde{V}\left(\partial_{t} w\right)^{2}-\int \partial_{t t} \widetilde{V} w \partial_{t} w .
\end{aligned}
$$

We next pick $0<\alpha<1$ close enough to 1 and combine the above identities to get

$$
\begin{aligned}
& \frac{1}{2 \lambda^{2 \alpha}} \frac{d}{d t}\left\{\lambda ^ { 2 \alpha } \left[\int\left(\partial_{t r} w\right)^{2}-\int \widetilde{V}\left(\partial_{t} w\right)^{2}+\int\right.\right.\left.\left.\left(H_{\lambda} w\right)^{2}-2 \int \partial_{t} \widetilde{V} w \partial_{t} w\right]\right\} \\
&=-R_{1}+R_{2}+\frac{2 \alpha b}{\lambda} \int \partial_{t} \widetilde{V} w \partial_{t} w-\int \partial_{t t} \widetilde{V} w \partial_{t} w,
\end{aligned}
$$

where $R_{1}$ collects the quadratic terms

$$
\begin{aligned}
R_{1} & =\frac{\alpha b}{\lambda}\left[\int\left(\partial_{t r} w\right)^{2}-\int \widetilde{V}\left(\partial_{t} w\right)^{2}+\int\left(H_{\lambda} w\right)^{2}\right]+\frac{3}{2} \int \partial_{t} \widetilde{V}\left(\partial_{t} w\right)^{2}-\frac{b_{s}}{\lambda^{2}} \int \partial_{t} \widetilde{V}(\Lambda Q)_{\lambda} w \\
& =\frac{b}{\lambda^{3}}\left[\alpha \int\left(\partial_{y} \eta\right)^{2}-\alpha \int V \eta^{2}+\alpha \int(H \varepsilon)^{2}+\frac{3}{2} \int(2 V+y \cdot \nabla V) \eta^{2}-b_{s} \int \varepsilon(2 V+y \cdot \nabla V) \Lambda Q\right]
\end{aligned}
$$


and $R_{2}$ collects the nonlinear higher-order terms

$$
R_{2}=\int \partial_{t} w H_{\lambda} F_{B_{1}}-\int \partial_{t} \widetilde{V} w\left[F_{B_{1}}+\frac{b_{s}}{\lambda^{2}}(\Lambda Q)_{\lambda}\right] .
$$

Step 2: Derivation of the quadratic terms and treatment of the $b_{s}$ term. Let us now obtain a suitable lower bound for the quadratic term $R_{1}$. The main enemy is the $b_{s}$ term which is of order one in $\varepsilon$ and will be treated by using a specific algebra generated by the choice of the orthogonality condition (3-4).

Observe from $H(\Lambda Q)=0$ that $(\Lambda Q / \lambda)_{\lambda}(y)=(1 / \lambda)^{N / 2}(\Lambda Q)(y / \lambda)$ satisfies

$$
-\Delta(\Lambda Q / \lambda)_{\lambda}(y)-(1 / \lambda)^{2} V(y / \lambda)(\Lambda Q / \lambda)_{\lambda}(y)=0 .
$$

Differentiating this relation at $\lambda=1$ yields

$$
H \Phi=H(D \Lambda Q)=(2 V+y \cdot \nabla V) \Lambda Q .
$$

We inject this into the modulation equation (4-8) to get

$$
-b_{s} \int \varepsilon(2 V+y \cdot \nabla V) \Lambda Q=b_{s}^{2}(\Phi, \Lambda Q)+\left|b_{s}\right| O\left(\frac{|\mathscr{E}|}{M}+\sqrt{K(M)} \frac{b^{4}}{|\log b|^{2}}\right)^{1 / 2} \text {. }
$$

We thus conclude using the sign

$$
(\Phi, \Lambda Q)>0
$$

and (4-8), (4-21) that

$$
\begin{aligned}
R_{1} \geq \frac{b}{\lambda^{3}}\left[\alpha \int\left(\partial_{y} \eta\right)^{2}+\int\left[(3-\alpha) V+\frac{3}{2} y \cdot \nabla V\right] \eta^{2}+\alpha \int(H \varepsilon)^{2}\right. & +c_{1}\left(b_{s}\right)^{2} \\
& \left.+O\left(\frac{|\mathscr{E}|}{M}+\sqrt{K(M)} \frac{b^{4}}{|\log b|^{2}}\right)\right]
\end{aligned}
$$

for some universal constant $c_{1}>0$ independent of $M$.

Step 3: Coercivity of the quadratic form. We now claim the following coercivity property of the quadratic form in $\eta$ appearing on the right-hand side of (4-24) in the limit case $\alpha=1$. The proof is given in Appendix B.

Lemma 4.7. There exists a universal constant $c_{0}>0$ such that for all $\eta \in \dot{H}_{\mathrm{rad}}^{1}$ we have

$$
\int\left(\partial_{y} \eta\right)^{2}+\int\left[2 V+\frac{3}{2} y \cdot \nabla V\right] \eta^{2} \geq c_{0} \int\left(\partial_{y} \eta\right)^{2}-\frac{1}{c_{0}}\left[(\eta, \psi)^{2}+(\eta, \Phi)^{2}\right] .
$$

From a simple continuity argument, there exists $0<\alpha^{*}<1$ such that given $0<\alpha^{*}<\alpha \leq 1$, for all $\eta \in \dot{H}_{\text {rad }}^{1}$, we have

$$
\alpha \int\left(\partial_{y} \eta\right)^{2}+\int\left[(3-\alpha) V+\frac{3}{2} y \cdot \nabla V\right] \eta^{2} \geq \frac{c_{0}}{2} \int\left(\partial_{y} \eta\right)^{2}-\frac{2}{c_{0}}\left[(\eta, \psi)^{2}+(\eta, \Phi)^{2}\right] .
$$

We now pick once and for all such an $\alpha<1$ and control the negative directions.

Using (4-3) and (4-7) yields

$$
(\eta, \psi)^{2} \lesssim b|\mathscr{E}|+\sqrt{K(M)} \frac{b^{4}}{|\log b|^{2}}
$$


Similarly, we compute $(\eta, \Phi)=\left(\eta, \chi_{M} \Phi\right)+\left(\eta,\left(1-\chi_{M}\right) \Phi\right)$ for which (4-4) and (4-7) yield

$$
\left(\eta, \chi_{M} \Phi\right)^{2} \lesssim b|\mathscr{E}|+\sqrt{K(M)} \frac{b^{4}}{|\log b|^{2}},
$$

and applying (C-1) we have

$$
\left(\eta,\left(1-\chi_{M}\right) \Phi\right)^{2} \leq\|y \eta\|_{L^{\infty}}^{2}\left[\int_{y \geq M / 2} \frac{|\Phi|}{y}\right]^{2} \lesssim \frac{1}{M} \int\left|\partial_{y} \eta\right|^{2} .
$$

This, together with (4-24), yields the lower bound on quadratic terms,

$$
R_{1} \geq \frac{b}{\lambda^{3}}\left[c_{1}\left(\left(b_{s}\right)^{2}+|\mathscr{E}|\right)+O\left(\sqrt{K(M)} \frac{b^{4}}{|\log b|^{2}}\right)\right]
$$

for some universal constant $c_{1}>0$. Indeed, a straightforward integration by parts in (3-19) yields

$$
\mathscr{E} \lesssim \int\left|\partial_{y} \eta\right|^{2}+\int|H \varepsilon|^{2}
$$

Step 4: Control of lower-order quadratic terms. The lower-order quadratic terms in (4-20) are controlled similarly,

$$
\begin{aligned}
\left|\int \partial_{t} \tilde{V} w \partial_{t} w\right| \lesssim \frac{b}{\lambda^{2}}\left[\int \frac{\varepsilon^{2}}{1+y^{6}}+\int \frac{\eta^{2}}{y^{2}}\right] & \lesssim \frac{1}{\lambda^{2}}\left(b C(M)|\mathscr{E}|+\sqrt{K(M)} \frac{b^{4}}{|\log b|^{2}}\right) \\
& \lesssim \frac{1}{\lambda^{2}}\left(\frac{|\mathscr{E}|}{M}+\sqrt{K(M)} \frac{b^{4}}{|\log b|^{2}}\right),
\end{aligned}
$$

and, with the help of (3-32),

$$
\left|\int \partial_{t t} \widetilde{V} w \partial_{t} w\right| \lesssim\left(\frac{b^{2}}{\lambda^{3}}+\frac{\left|b_{s}\right|}{\lambda^{3}}\right)\left[\int \frac{\varepsilon^{2}}{1+y^{6}}+\int \frac{\eta^{2}}{y^{2}}\right] \lesssim \frac{b}{\lambda^{3}}\left(b C(M)|\mathscr{E}|+\sqrt{K(M)} \frac{b^{4}}{|\log b|^{2}}\right) .
$$

Remark 4.8. We note here that (4-26) is sufficient for the proof of our theorem. Indeed, the estimated term $\int \partial_{t} \widetilde{V} w \partial_{t} w$ has been integrated by parts with respect to time, so that it becomes a part of $\mathscr{F}$. Furthermore, we note that to compute (4-16), we multiply $\mathscr{F}$ by $\lambda^{2 \alpha}$. Consequently, the commutator $b \alpha / \lambda \int \partial_{t} \widetilde{V} w \partial_{t} w$ appears on the right-hand side. However, (4-26) yields that, in the trapped regime, this supplementary term is controlled by $b / \lambda^{3} \sqrt{K(M)} b^{4} /|\log b|^{2}$. Similar arguments will be repeated implicitly below for the terms that require an integration by parts with respect to time.

Step 5: Rewriting the nonlinear $R_{2}$ terms. It remains to control the nonlinear $R_{2}$ terms in (4-20) given by (4-22). According to (3-17), this term contains $b_{s s}$-types of terms which cannot be estimated in absolute value and require a further integration by parts in time. Let

$$
F_{B_{1}}=F_{1}-\partial_{t} F_{2}, \quad \text { with } F_{2}=\frac{1}{\lambda}\left(\partial_{s} P_{B_{1}}\right)_{\lambda},
$$

and write

$$
R_{2}=\int \partial_{t} w H_{\lambda} F_{1}-\int \partial_{t} \tilde{V} w\left[F_{1}+\frac{b_{s}}{\lambda^{2}}(\Lambda Q)_{\lambda}\right]-\int \partial_{t} w H_{\lambda} \partial_{t} F_{2}+\int \partial_{t} \widetilde{V} w \partial_{t} F_{2}
$$


We now integrate by parts in time to treat the $F_{2}$ term,

$$
\begin{aligned}
-\int \partial_{t} w H_{\lambda} \partial_{t} F_{2}+\int \partial_{t} \widetilde{V} w \partial_{t} F_{2} & \\
& =-\frac{d}{d t}\left\{\int \partial_{t} w H_{\lambda} F_{2}-\int \partial_{t} \widetilde{V} w F_{2}\right\}-\int\left(\partial_{t t} \widetilde{V} w+2 \partial_{t} \widetilde{V} \partial_{t} w\right) F_{2}+\int \partial_{t t} w H_{\lambda} F_{2} .
\end{aligned}
$$

The last term is rewritten using (3-17) and integration by parts,

$$
\begin{aligned}
\int \partial_{t t} w H_{\lambda} F_{2} & =\int\left[F_{1}-\partial_{t} F_{2}-H_{\lambda} w\right] H_{\lambda} F_{2} \\
& =-\frac{1}{2} \frac{d}{d t}\left\{\int\left|\nabla F_{2}\right|^{2}-\int \widetilde{V} F_{2}^{2}\right\}-\frac{1}{2} \int \partial_{t} \widetilde{V} F_{2}^{2}+\int\left[F_{1}-H_{\lambda} w\right] H_{\lambda} F_{2} .
\end{aligned}
$$

Eventually we arrive at a manageable expression for $R_{2}$,

$$
\begin{array}{r}
R_{2}=-\frac{d}{d t}\left\{\int \partial_{t} w H_{\lambda} F_{2}-\int \partial_{t} \tilde{V} w F_{2}+\frac{1}{2} \int\left|\nabla F_{2}\right|^{2}-\frac{1}{2} \int \tilde{V} F_{2}^{2}\right\} \\
-\int \partial_{t} \widetilde{V} w\left[F_{1}+\frac{b_{s}}{\lambda^{2}}(\Lambda Q)_{\lambda}\right]+\int \partial_{t} w H_{\lambda} F_{1}-\int\left(\partial_{t} \tilde{V} w+2 \partial_{t} \widetilde{V} \partial_{t} w\right) F_{2} \\
-\frac{1}{2} \int \partial_{t} \widetilde{V} F_{2}^{2}+\int\left[F_{1}-H_{\lambda} w\right] H_{\lambda} F_{2} .
\end{array}
$$

We now aim at estimating all the terms in the right-hand side of (4-28). According to (3-17), we split $F_{1}$ into four terms

$$
F_{1}+\frac{b_{s}}{\lambda^{2}}(\Lambda Q)_{\lambda}=-\frac{1}{\lambda^{2}}\left[\Psi_{B_{1}}+F_{1,1}+F_{1,2}+N(\varepsilon)\right]_{\lambda}
$$

with

$$
F_{1,1}=b \Lambda \partial_{s} P_{B_{1}}+b_{s}\left(\Lambda P_{B_{1}}-\Lambda Q\right), \quad F_{1,2}=\left[f^{\prime}(Q)-f^{\prime}\left(P_{B_{1}}\right)\right] \varepsilon
$$

Step 6: $F_{1}$ terms. These are the leading-order terms.

- $\Psi_{B_{1}}$ terms. We first extract from (2-9) the rough bound

which yields

$$
\left|\Psi_{B_{1}}\right| \lesssim \frac{b^{2}}{|\log b|\left(1+y^{2}\right)}+C(M) b^{4} \mathbf{1}_{y \leq 2 B_{1}},
$$

$$
\int \frac{1+|\log y|^{2}}{1+y^{4}}\left|\Psi_{B_{1}}\right|^{2} \lesssim \frac{b^{4}}{|\log b|^{2}}
$$

and thus, from (4-7),

$$
\begin{aligned}
\left|\int \partial_{t} \widetilde{V} w \frac{1}{\lambda^{2}}\left(\Psi_{B_{1}}\right)_{\lambda}\right| & \lesssim \frac{b}{\lambda^{3}} \int \frac{|\varepsilon|\left|\Psi_{B_{1}}\right|}{\left(1+y^{4}\right)} \\
& \lesssim \frac{b}{\lambda^{3}} \frac{b^{2}}{|\log b|} C(M) \sqrt{|\mathscr{E}|+\sqrt{K(M)} b^{4} /|\log b|^{2}} \\
& \lesssim \frac{b}{\lambda^{3}} \sqrt{K(M)} \frac{b^{4}}{|\log b|^{2}} .
\end{aligned}
$$


Next we use the fundamental cancellation $H(\Lambda Q)=0$ and (2-9) to estimate

$$
\begin{array}{r}
\left|H \Psi_{B_{1}}\right| \lesssim \frac{b^{4}}{1+y^{2}}\left[\frac{1+|\log (b y)|}{|\log b|} \mathbf{1}_{2 \leq y \leq 2 B_{0}}+\frac{1}{b^{2} y^{2}|\log b|} \mathbf{1}_{B_{0} / 2 \leq y \leq 2 B_{1}}+\frac{\log (M)+|\log (1+y)|}{1+y^{2}} \mathbf{1}_{y \leq 2 B_{1}}\right] \\
+\frac{b^{2}}{\left(1+y^{4}\right)|\log b|} \mathbf{1}_{y \geq B_{1} / 2},
\end{array}
$$

and thus get

$$
\int\left(1+y^{2}\right)\left|H\left(\Psi_{B_{1}}\right)\right|^{2} \lesssim \frac{b^{6}}{|\log b|^{2}}
$$

Hence

$$
\left|\int \partial_{t} w H_{\lambda}\left(\frac{1}{\lambda^{2}}\left(\Psi_{B_{1}}\right)_{\lambda}\right)\right| \lesssim \frac{b}{\lambda^{3}}\|\eta / y\|_{L^{2}}\left[\int \frac{1}{b^{2}}(1+y)^{2}\left|H\left(\Psi_{B_{1}}\right)\right|^{2}\right]^{1 / 2} \lesssim \frac{b}{\lambda^{3}} \sqrt{K(M)} \frac{b^{4}}{|\log b|^{2}} .
$$

- $F_{1,1}$ terms. From (2-7) and (2-8) we obtain

$$
\left|F_{1,1}\right| \lesssim\left|b_{s}\right| b^{2}\left[\frac{1+|\log (b y)|}{|\log b|} \mathbf{1}_{2 \leq y \leq B_{0} / 2}+\frac{1}{b^{2} y^{2}|\log b|} \mathbf{1}_{B_{0} / 2 \leq y \leq 2 B_{1}}+\frac{\log (M)+|\log y|}{1+y^{2}}\right],
$$

and, recalling that differentiation with respect to $y$ acts as a multiplication by $1 /(1+y)$,

$$
\left|H F_{1,1}\right| \lesssim C(M) \frac{\left|b_{s}\right| b^{2}}{1+y^{2}}\left[\frac{1+|\log (b y)|}{|\log b|} \mathbf{1}_{2 \leq y \leq B_{0} / 2}+\frac{1}{b^{2} y^{2}|\log b|} \mathbf{1}_{B_{0} / 2 \leq y \leq 2 B_{1}}+\frac{\log (M)+|\log y|}{1+y^{2}}\right],
$$

from which

$$
\int\left(1+y^{2}\right)\left|H\left(F_{1,1}\right)\right|^{2} \lesssim\left|b_{s}\right|^{2} \frac{b^{2}}{|\log b|^{2}}, \quad \int \frac{\left(1+|\log y|^{2}\right)}{\left(1+y^{4}\right)}\left|F_{1,1}\right|^{2} \lesssim\left|b_{s}\right|^{2} b^{2} .
$$

Hence similar arguments as with the $\Psi_{B_{1}}$ terms yield

$$
\left|\int \partial_{t} \widetilde{V} w F_{1,1}\right| \lesssim \frac{b}{\lambda^{3}} b\left|b_{s}\right| C(M) \sqrt{|\mathscr{E}|+\sqrt{K(M)} b^{4} /|\log b|^{2}} \lesssim \frac{b}{\lambda^{3}} \sqrt{K(M)} \frac{b^{4}}{|\log b|^{2}},
$$

and

$$
\begin{aligned}
\left|\int \partial_{t} w H_{\lambda} F_{1,1}\right| & \lesssim \frac{C(M) b}{\lambda^{3}} \frac{\left|b_{s}\right|}{|\log b|} \sqrt{|\mathscr{E}|+\sqrt{K(M)} b^{4} /|\log b|^{2}} \\
& \lesssim \frac{b}{\lambda^{3}}\left[\frac{\left|b_{s}\right|^{2}}{|\log b|}+\frac{\mathscr{E}}{|\log b|}+\sqrt{K(M)} \frac{b^{4}}{|\log b|^{2}}\right] \lesssim \frac{b}{\lambda^{3}} \sqrt{K(M)} \frac{b^{4}}{|\log b|^{2}} .
\end{aligned}
$$

- $F_{1,2}$ terms. The explicit expansion of the cubic nonlinearity and the bound (2-7) yield

$$
\left|F_{1,2}\right| \lesssim \frac{C(M) b^{2}}{1+y^{2}}|\varepsilon| \quad \text { and } \quad\left|\nabla F_{1,2}\right| \lesssim \frac{C(M) b^{2}}{1+y^{3}}|\varepsilon|+\frac{C(M) b^{2}}{1+y^{2}}|\nabla \varepsilon|
$$

from which

$$
\frac{1}{\lambda^{2}}\left|\int \partial_{t} \tilde{V} w\left(F_{1,2}\right)_{\lambda}\right| \lesssim \frac{C(M) b^{3}}{\lambda^{3}} \int \frac{\varepsilon^{2}}{1+y^{6}} \lesssim \frac{b}{\lambda^{3}}\left(b|\mathscr{E}|+\sqrt{K(M)} \frac{b^{4}}{|\log b|^{2}}\right),
$$


and, after integration by parts of the Laplacian term,

$$
\begin{aligned}
\frac{1}{\lambda^{2}}\left|\int \partial_{t} w H_{\lambda}\left(F_{1,2}\right)_{\lambda}\right| & \lesssim \frac{C(M)}{\lambda^{3}}\left[\int \frac{|\eta|}{1+y^{4}} \frac{b^{2}}{1+y^{2}}|\varepsilon|+\int|\nabla \eta|\left(\frac{b^{2}}{1+y^{3}}|\varepsilon|+\frac{b^{2}}{1+y^{2}}|\nabla \varepsilon|\right)\right] \\
& \lesssim \frac{b}{\lambda^{3}}\left[\frac{|\mathscr{E}|}{M}+\sqrt{K(M)} \frac{b^{4}}{|\log b|^{2}}\right] .
\end{aligned}
$$

- Nonlinear term $N(\varepsilon)$. We expand the nonlinearity as

$$
N(\varepsilon)=3 P_{B_{1}} \varepsilon^{2}+\varepsilon^{3} .
$$

This yields, using (3-27) and (C-1), the rough bound

$$
|N(\varepsilon)| \lesssim \frac{\varepsilon^{2}}{1+y}
$$

In what follows, we will use the following bound on $\eta$, which follows from (4-6), (C-1):

$$
\|y \eta\|_{L^{\infty}} \lesssim\|\nabla \eta\|_{L^{2}} \lesssim\left(c(M)|\mathscr{E}|+\sqrt{K(M)} \frac{b^{4}}{|\log b|^{2}}\right)^{1 / 2}
$$

We then estimate

$$
\left|\frac{1}{\lambda^{2}} \int \partial_{t} \tilde{V} w(N(\varepsilon))_{\lambda}\right| \lesssim \frac{b}{\lambda^{3}} \int \frac{|\varepsilon|^{3}}{1+y^{5}} \lesssim \frac{b}{\lambda^{3}}\|\nabla \varepsilon\|_{L^{2}}\left(c(M)|\mathscr{E}|+\sqrt{K(M)} \frac{b^{4}}{|\log b|^{2}}\right) \lesssim \frac{b}{\lambda^{3}} \sqrt{K(M)} \frac{b^{4}}{|\log b|^{2}}
$$

for $b_{0}<b^{*}(M)$ small enough. We split the second term into

$$
\int \partial_{t} w H_{\lambda}\left(\frac{(N(\varepsilon))_{\lambda}}{\lambda^{2}}\right)=\int \nabla \partial_{t} w \cdot \nabla\left(\frac{(N(\varepsilon))_{\lambda}}{\lambda^{2}}\right)-\int \widetilde{V} \partial_{t} w\left(\frac{(N(\varepsilon))_{\lambda}}{\lambda^{2}}\right)
$$

The second of these terms is estimated by brute force:

$$
\begin{aligned}
\left|\int \tilde{V} \partial_{t} w\left(\frac{(N(\varepsilon))_{\lambda}}{\lambda^{2}}\right)\right| \lesssim \frac{1}{\lambda^{3}} \int \frac{|\eta||\varepsilon|^{2}}{1+y^{5}} & \lesssim \frac{1}{\lambda^{3}}\|y \eta\|_{L^{\infty}} \int \frac{|\varepsilon|^{2}}{1+y^{6}} \\
& \lesssim \frac{1}{\lambda^{3}}\left(c(M)|\mathscr{E}|+\sqrt{K(M)} \frac{b^{4}}{|\log b|^{2}}\right)^{3 / 2} \lesssim \frac{b}{\lambda^{3}} \frac{b^{4}}{|\log b|^{2}} .
\end{aligned}
$$

The first term in (4-35) is split into two parts:

$$
\int \nabla \partial_{t} w \cdot \nabla\left(\frac{(N(\varepsilon))_{\lambda}}{\lambda^{2}}\right)=\int \nabla \partial_{t} w \cdot\left[\nabla\left(w^{3}\right)+3\left(P_{B_{1}}\right)_{\lambda} \nabla\left(w^{2}\right)\right]+\frac{3}{\lambda^{3}} \int \varepsilon^{2} \nabla \eta \cdot \nabla P_{B_{1}} .
$$

The second term is integrated by parts in space and then estimated by brute force: 


$$
\begin{aligned}
\left|\frac{3}{\lambda^{3}} \int \varepsilon^{2} \nabla \eta \cdot \nabla P_{B_{1}}\right| & =\frac{3}{\lambda^{3}}\left|\int \eta\left[\varepsilon^{2} \Delta P_{B_{1}}+2 \varepsilon \nabla P_{B_{1}} \cdot \nabla \varepsilon\right]\right| \\
& \lesssim \frac{1}{\lambda^{3}} \int|\eta|\left[\frac{\varepsilon^{2}}{1+y^{4}}+\frac{|\varepsilon||\nabla \varepsilon|}{1+y^{3}}\right] \\
& \lesssim \frac{1}{\lambda^{3}}\|y \eta\|_{L^{\infty}}\left[\int \frac{\varepsilon^{2}}{1+y^{5}}+\int \frac{|\nabla \varepsilon|^{2}}{y^{2}}\right] \\
& \lesssim \frac{1}{\lambda^{3}}\left(c(M)|\mathscr{E}|+\sqrt{K(M)} \frac{b^{4}}{|\log b|^{2}}\right)^{3 / 2} \lesssim \frac{b}{\lambda^{3}} \frac{b^{4}}{|\log b|^{2}} .
\end{aligned}
$$

The first term is more delicate and requires first a time integration by parts,

$$
\begin{aligned}
\int \nabla \partial_{t} w \cdot\left[\nabla\left(w^{3}\right)+3\left(P_{B_{1}}\right)_{\lambda} \nabla\left(w^{2}\right)\right]=\frac{d}{d t}\left\{\int\right. & \left.|\nabla w|^{2}\left[\frac{3}{2} w^{2}+3\left(P_{B_{1}}\right)_{\lambda} w\right]\right\} \\
& -3 \int w \partial_{t} w|\nabla w|^{2}-3 \int|\nabla w|^{2}\left[w \partial_{t}\left(P_{B_{1}}\right)_{\lambda}+\left(P_{B_{1}}\right)_{\lambda} \partial_{t} w\right]
\end{aligned}
$$

We may now estimate all terms by brute force. First,

$$
\left.\left|\int\right| \nabla w\right|^{2}\left[\frac{3}{2} w^{2}+3\left(P_{B_{1}}\right)_{\lambda} w\right] \mid \lesssim \frac{1}{\lambda^{2}}\left[\|y \varepsilon\|_{L^{\infty}}+\left\|y P_{B_{1}}\right\|_{L^{\infty}}\right]\|y \varepsilon\|_{L^{\infty}} \int \frac{|\nabla \varepsilon|^{2}}{y^{2}} \lesssim \frac{1}{\lambda^{2}} \frac{b^{4}}{|\log b|^{2}},
$$

second,

$$
\left.\left|\int w \partial_{t} w\right| \nabla w\right|^{2} \mid \lesssim \frac{1}{\lambda^{2}}\|y \varepsilon\|_{L^{\infty}}\|y \eta\|_{L^{\infty}} \int \frac{|\nabla \varepsilon|^{2}}{y^{2}} \lesssim\left(c(M)|\mathscr{E}|+\sqrt{K(M)} \frac{b^{4}}{|\log b|^{2}}\right)^{3 / 2} \lesssim \frac{b}{\lambda^{3}} \frac{b^{4}}{|\log b|^{2}},
$$

and third,

$$
\begin{aligned}
\left.\left|\int\right| \nabla w\right|^{2} w \partial_{t}\left(P_{B_{1}}\right)_{\lambda} \mid & \lesssim \frac{\|y w\|_{L^{\infty}}}{\lambda^{3}} \int \frac{|\nabla w|^{2}}{y}\left[\frac{b}{1+y^{2}}+C(M) b\left|b_{s}\right| \mathbf{1}_{y \leq B_{1}}\right] \\
& \lesssim \frac{b}{\lambda^{3}}|\nabla \varepsilon|_{L^{2}}\left(1+C(M)\left|b_{s}\right| \frac{|\log b|}{b}\right) \int \frac{|\nabla \varepsilon|^{2}}{y^{2}} \lesssim \frac{b}{\lambda^{3}} \frac{b^{4}}{|\log b|^{2}},
\end{aligned}
$$

where we used the rough bound extracted from (2-8), $\left|\partial_{b} P_{B_{1}}\right| \lesssim C(M) b \mathbf{1}_{y \leq B_{1}}$. Finally,

$$
\left.\left|\int\right| \nabla w\right|^{2}\left(P_{B_{1}}\right)_{\lambda} \partial_{t} w \mid \lesssim \frac{1}{\lambda^{3}}\|y \eta\|_{L^{\infty}} \int \frac{|\nabla \varepsilon|^{2}}{1+y^{3}} \lesssim\left(C(M)^{\mathscr{E}}+\sqrt{K(M)} \frac{b^{4}}{|\log b|^{2}}\right)^{3 / 2} \lesssim \frac{b}{\lambda^{3}} \frac{b^{4}}{|\log b|^{2}},
$$

for $b_{0}<b^{*}(M)$ small enough. The above chain of estimates together with Remark 4.8, achieves the control of the nonlinear term $N(\varepsilon)$.

Step 7: $F_{2}$ terms. We estimate from (2-8),

$$
\int\left|\frac{\partial_{b} P_{B_{1}}}{(1+y)}\right|^{2}+\int\left|\nabla \partial_{b} P_{B_{1}}\right|^{2} \lesssim \frac{1}{|\log b|^{2}} \quad \text { and } \quad \int \frac{1}{1+y^{3}}\left|\partial_{b} P_{B_{1}}\right|^{2} \lesssim \frac{b}{|\log b|^{2}}
$$


Hence, first,

$$
\begin{aligned}
\left|\int \partial_{t} w H_{\lambda} F_{2}\right| & \lesssim \frac{\left|b_{s}\right|}{\lambda^{2}}\left[\int \frac{|\eta|\left|\partial_{b} P_{B_{1}}\right|}{\left(1+y^{4}\right)}+\int|\nabla \eta|\left|\nabla \partial_{b} P_{B_{1}}\right|\right] \\
& \lesssim \frac{1}{\lambda^{2}} \frac{\left|b_{s}\right|}{|\log b|} \sqrt{c(M)|\mathscr{E}|+\sqrt{K(M)} b^{4} /|\log b|^{2}} \\
& \lesssim \frac{1}{\lambda^{2}}\left[\frac{|\mathscr{E}|}{M}+\sqrt{K(M)} \frac{b^{4}}{|\log b|^{2}}\right],
\end{aligned}
$$

second,

$$
\begin{aligned}
\left|\int \partial_{t} \widetilde{V} w F_{2}\right| \lesssim \frac{\left|b_{s}\right| b}{\lambda^{2}} \int \frac{\left|\partial_{b} P_{B_{1}}\right||\varepsilon|}{\left(1+y^{4}\right)} & \lesssim \frac{\left|b_{s}\right| b}{\lambda^{2}|\log b|}\left[c(M)|\mathscr{E}|+\sqrt{K(M)} \frac{b^{4}}{|\log b|^{2}}\right]^{\frac{1}{2}} \\
& \lesssim \frac{1}{\lambda^{2}}\left[\frac{|\mathscr{E}|}{M}+\sqrt{K(M)} \frac{b^{4}}{|\log b|^{2}}\right]
\end{aligned}
$$

and third,

$$
\int\left|\nabla F_{2}\right|^{2}+\left|\int V F_{2}^{2}\right| \lesssim \frac{\left|b_{s}\right|^{2}}{\lambda^{2}}\left[\int \frac{\left|\partial_{b} P_{B_{1}}\right|^{2}}{\left(1+y^{4}\right)}+\int\left|\nabla \partial_{b} P_{B_{1}}\right|^{2}\right] \lesssim \frac{1}{\lambda^{2}} \frac{\left(b_{s}\right)^{2}}{|\log b|^{2}} \lesssim \frac{1}{\lambda^{2}} \frac{b^{4}}{|\log b|^{2}}
$$

Similarly,

$$
\begin{aligned}
\left|\int\left(\partial_{t} \tilde{V} w+2 \partial_{t} \tilde{V} \partial_{t} w\right) F_{2}\right|+\mid \int \partial_{t} \tilde{V} & F_{2}^{2} \mid \\
& \lesssim \frac{\left|b_{s}\right|}{\lambda^{3}}\left[\int \frac{\left(\left(\left|b_{s}\right|+b^{2}\right)|\varepsilon|+b|\eta|\right)\left|\partial_{b} P_{B_{1}}\right|}{\left(1+y^{4}\right)}+\left|b_{s}\right| b \int \frac{\left|\partial_{b} P_{B_{1}}\right|^{2}}{1+y^{4}}\right] \\
& \lesssim \frac{\left|b_{s}\right|}{\lambda^{3}}\left[\frac{\left(\left|b_{s}\right|+b\right)}{|\log b|} \sqrt{c(M)|\mathscr{E}|+\sqrt{K(M)} b^{4} /|\log b|^{2}}+\frac{b^{2}}{|\log b|^{2}}\left|b_{s}\right|\right] \\
& \lesssim \frac{b}{\lambda^{3}}\left[\frac{|\mathscr{E}|}{M}+\sqrt{K(M)} \frac{b^{4}}{|\log b|^{2}}\right] .
\end{aligned}
$$

Eventually, (4-32) and (4-33) ensure that

$$
\int\left(1+y^{2}\right)\left|H\left(\Psi_{B_{1}}+F_{1,1}\right)\right|^{2} \lesssim\left[\frac{b^{6}}{|\log b|^{2}}+\frac{b^{2}\left|b_{s}\right|^{2}}{|\log b|^{2}}\right] \lesssim \frac{b^{6}}{|\log b|^{2}}
$$

which together with (4-36) yields

$$
\left|\int \frac{1}{\lambda^{2}}\left(\Psi_{B_{1}}+F_{1,1}\right)_{\lambda} H_{\lambda} F_{2}\right| \lesssim \frac{1}{\lambda^{3}} \frac{b^{3}\left|b_{s}\right|}{|\log b|^{2}} \lesssim \frac{b}{\lambda^{3}} \frac{b^{4}}{|\log b|^{2}} .
$$

We similarly estimate from (4-34), after integration by parts, that

$$
\begin{aligned}
\left|\int \frac{1}{\lambda^{2}}\left(F_{1,2}\right)_{\lambda} H_{\lambda} F_{2}\right| & \lesssim \frac{\left|b_{s}\right|}{\lambda^{3}}\left[\int \frac{b^{2}|\varepsilon|\left|\partial_{b} P_{B_{1}}\right|}{1+y^{6}}+\int\left|\nabla \partial_{b} P_{B_{1}}\right|\left(\frac{b^{2}|\varepsilon|}{1+y^{3}}+\frac{b^{2}|\nabla \varepsilon|}{1+y^{2}}\right)\right] \\
& \lesssim C(M) \frac{b^{4}}{\lambda^{3}|\log b|}\left(\int \frac{\varepsilon^{2}}{1+y^{6}}+\int \frac{|\nabla \varepsilon|^{2}}{1+y^{4}}\right)^{1 / 2} \lesssim \frac{b}{\lambda^{3}} \frac{b^{4}}{|\log b|^{2}} .
\end{aligned}
$$


For the nonlinear term, we extract from (2-8) the rough bound

$$
\left|H\left(\partial_{b} P_{B_{1}}\right)\right| \lesssim[C(M)+\log (b)] \frac{b}{1+y^{2}} \mathbf{1}_{y \leq B_{1}},
$$

which together with $(\mathrm{C}-1)$ ensures that

$$
\begin{aligned}
\left|\int \frac{1}{\lambda^{2}}(N(\varepsilon))_{\lambda} H_{\lambda} F_{2}\right| & \lesssim \frac{[C(M)+\log (b)]}{\lambda^{3}}\left|b_{s}\right| \int \frac{b}{1+y^{2}} \frac{\varepsilon^{2}}{1+y} \mathbf{1}_{y \leq B_{1}} \\
& \lesssim C(M) \frac{\left|b_{s}\right||\log b|^{4}}{\lambda^{3}} \int \frac{\varepsilon^{2}}{\left(1+y^{4}\right)|\log y|^{2}} \\
& \lesssim \frac{b}{\lambda^{3}} \sqrt{b}\left(c(M)|\mathscr{E}|+\sqrt{K(M)} \frac{b^{4}}{|\log b|^{2}}\right) \lesssim \frac{b}{\lambda^{3}} \frac{b^{4}}{|\log b|^{2}} .
\end{aligned}
$$

Step 10: The remaining $F_{2}$ term has the right sign. It remains to estimate the term

$$
-\int H_{\lambda} w H_{\lambda} F_{2}
$$

on the right-hand side of (4-28). Let us stress the fact that this term is a priori no better $O\left(\mathscr{E} / \lambda^{3}\right)$ due to the $b_{s}$ contribution and the bound (4-8); recall Remark 4.5.

We now claim that the main contribution has the right sign again. Indeed, we first compute from the $T_{1}$ equation (2-16) that

$$
H T_{1}=-\Phi+c_{b} \chi_{B_{0} / 4} \Lambda Q \quad \text { and } \quad H \partial_{b} T_{1}=O\left(\frac{1}{b|\log b|} \frac{\mathbf{1}_{2 \leq y \leq B_{0} / 2}}{\left(1+y^{2}\right)}\right) .
$$

We then apply the decomposition (2-22),

$$
H\left(\partial_{b} P_{B_{1}}\right)=H\left(2 b T_{1}+2 b\left(\chi_{B_{1}}-1\right) T_{1}+b^{2} \partial_{b} \log \left(B_{1}\right) \rho_{B_{1}} T_{1}+b^{2} \chi_{B_{1}} \partial_{b} T_{1}\right)=-2 b \Phi+\Sigma,
$$

and estimate using (2-8), (2-21), (4-37) that

$$
|\Sigma| \lesssim \frac{b}{1+y^{2}}\left[\frac{1}{|\log b|} \mathbf{1}_{2 \leq y \leq B_{0} / 2}+\frac{1}{b^{2} y^{2}|\log b|} \mathbf{1}_{B_{0} / 2 \leq y}\right] .
$$

In particular, $\int \Sigma^{2} \lesssim b^{2} /|\log b|$, and thus using the modulation equation (4-8) gives

$$
\begin{aligned}
-\int H_{\lambda} w H_{\lambda} F_{2} & =-\frac{b_{s}}{\lambda^{3}} \int(H \varepsilon) H\left(\partial_{b} P_{B_{1}}\right) \\
& =-\frac{b_{s}}{\lambda^{3}} \int H \varepsilon(-2 b \Phi+\Sigma) \\
& =2 \frac{b}{\lambda^{3}} b_{s}(\varepsilon, H \Phi)+\frac{b}{\lambda^{3}} O\left(\frac{\left|b_{s}\right|}{\sqrt{|\log b|}} \sqrt{|\mathscr{E}|+\sqrt{K(M)} b^{4} /|\log b|^{2}}\right) \\
& =2 \frac{b}{\lambda^{3}}\left[-\frac{(\varepsilon, H \Phi)}{(\Lambda Q, \Phi)}+O\left(\sqrt{|\mathscr{E}| / M+\sqrt{K(M)} b^{4} /|\log b|^{2}}\right)\right](\varepsilon, H \Phi)+\frac{b}{\lambda^{3}} O\left(\frac{b^{4}}{|\log b|^{2}}\right) \\
& =-\frac{2 b}{\lambda^{3}} \frac{(\varepsilon, H \Phi)^{2}}{(\Lambda Q, \Phi)}+O\left(\frac{|\mathscr{E}|}{M}+\sqrt{K(M)} \frac{b^{4}}{|\log b|^{2}}\right)+\frac{b}{\lambda^{3}} O\left(\frac{b^{4}}{|\log b|^{2}}\right)
\end{aligned}
$$




$$
\leq O\left(\frac{|\mathscr{E}|}{M}+\sqrt{K(M)} \frac{b^{4}}{|\log b|^{2}}\right)
$$

Collecting all the above estimates yields (4-16) and concludes the proof of Proposition 4.6.

Improved bound. We now claim that the a priori bound on the unstable direction (3-35), coupled with the monotonicity property of Proposition 4.6, implies the following:

Lemma 4.9 (improved bounds under the a priori control (3-35)). In $\left[0, T_{1}\left(a_{+}\right)\right]$we have

$$
\begin{aligned}
&\left\|\nabla w(t), \partial_{t} w(t)+\frac{b(t)}{\lambda(t)}\left(\left(1-\chi_{B_{1}(b(t))}\right) \Lambda Q\right)_{\lambda(t)}\right\|_{L^{2} \times L^{2}} \lesssim b_{0}\left|\log b_{0}\right|, \\
& \frac{b^{4}(t)}{|\log b(t)|^{2} \lambda^{2(1-\alpha)}(t)} \geq \frac{b^{4}(0)}{|\log b(0)|^{2} \lambda^{2(1-\alpha)}(0)}, \\
&\left|b_{s}\right|^{2} \leq \frac{K(M)}{2} \frac{b^{4}}{|\log b|^{2}}, \\
&|\mathscr{E}(t)| \leq \frac{K(M)}{2} \frac{b^{4}}{(\log b)^{2}} .
\end{aligned}
$$

Proof.

Step 1: Energy bound. The energy bound (4-40) is a consequence of the conservation of energy. Indeed, conservation of energy and the initial bounds of Lemma 3.1 ensure that

$$
E\left(u, \partial_{t} u\right)=E\left(u_{0}, u_{1}\right)=E(Q)+O\left(b_{0} \sqrt{\left|\log b_{0}\right|}\right)
$$

(see Appendix A) and thus give

$$
E(Q)+O\left(b_{0}\left|\log b_{0}\right|\right)=\frac{1}{2} \int\left[\partial_{t}\left(P_{B_{1}}\right)_{\lambda}+\partial_{t} w\right]^{2}+\frac{1}{2} \int\left|\nabla\left(P_{B_{1}}\right)_{\lambda}+\nabla w\right|^{2}-\frac{1}{4} \int\left[\left(P_{B_{1}}\right)_{\lambda}+w\right]^{4} .
$$

We lower bound the first term by expanding

$$
\begin{aligned}
\partial_{t}\left(P_{B_{1}}\right)_{\lambda}+\partial_{t} w & =\partial_{t} w+\frac{b}{\lambda}\left(\left(1-\chi_{B_{1}}\right) \Lambda Q\right)_{\lambda}+\frac{b}{\lambda}\left(\chi_{B_{1}} \Lambda Q\right)_{\lambda}+\frac{b^{3}}{\lambda}\left(\Lambda\left[\chi_{B_{1}} T_{1}\right]\right)_{\lambda}+\frac{b_{s}}{\lambda}\left(\partial_{b} P_{B_{1}}\right)_{\lambda} \\
& =\partial_{t} w+\frac{b}{\lambda}\left(\left(1-\chi_{B_{1}}\right) \Lambda Q\right)_{\lambda}+\Sigma
\end{aligned}
$$

with

$$
\int \Sigma^{2} \lesssim b_{0}^{2}\left|\log b_{0}\right|
$$

where we used the bootstrap bounds (3-31) and (3-32). Finally,

$$
\int\left[\partial_{t}\left(P_{B_{1}}\right)_{\lambda}+\partial_{t} w\right]^{2} \geq \frac{1}{2} \int\left[\frac{b}{\lambda}\left(\left(1-\chi_{B_{1}}\right) \Lambda Q\right)_{\lambda}+\partial_{t} w\right]^{2}-O\left(b_{0}^{2}\left|\log b_{0}\right|\right) .
$$


We then expand the second term as

$$
\begin{aligned}
\frac{1}{2} \int & {\left[\nabla\left(P_{B_{1}}\right)_{\lambda}+\nabla w\right]^{2}-\frac{1}{4} \int\left[\left(P_{B_{1}}\right)_{\lambda}+w\right]^{4} } \\
= & \frac{1}{2} \int\left[\nabla P_{B_{1}}+\nabla \varepsilon\right]^{2}-\frac{1}{4} \int\left[P_{B_{1}}+\varepsilon\right]^{4} \\
= & \frac{1}{2} \int\left|\nabla P_{B_{1}}\right|^{2}-\frac{1}{4} \int\left|P_{B_{1}}\right|^{4}-\left(\varepsilon, \Delta P_{B_{1}}+P_{B_{1}}^{3}\right)+\frac{1}{2}\left(\int|\nabla \varepsilon|^{2}-3 \int P_{B_{1}}^{2} \varepsilon^{2}\right)-\frac{1}{4}\left(4 P_{B_{1}} \varepsilon^{3}+\varepsilon^{4}\right) .
\end{aligned}
$$

From the construction of $P_{B_{1}}$,

$$
\frac{1}{2} \int\left|\nabla P_{B_{1}}\right|^{2}-\frac{1}{4} \int\left|P_{B_{1}}\right|^{4}=E(Q)+O\left(b^{2}|\log b|\right) .
$$

The linear term is treated using (2-9), the improved decay (2-2) and (4-31). We get

$$
\left|\left(\varepsilon, \Delta P_{B_{1}}+P_{B_{1}}^{3}\right)\right|=\left|\left(\varepsilon, b^{2} D \Lambda P_{B_{1}}-\Psi_{B_{1}}\right)\right| \lesssim\|\varepsilon / y\|_{L^{2}}\left\|y\left(b^{2} D \Lambda P_{B_{1}}-\Psi_{B_{1}}\right)\right\|_{L^{2}} \lesssim b|\nabla \varepsilon|_{L^{2}} .
$$

We now rewrite the quadratic term as a small deformation of $H$ and use the coercivity bound (C-8) to ensure that

$$
\int|\nabla \varepsilon|^{2}-3 \int P_{B_{1}}^{2} \varepsilon^{2} \geq c_{0} \int|\nabla \varepsilon|^{2}+D e f
$$

with

$$
\text { Def }:=3 \int\left(Q^{2}-P_{B_{1}}^{2}\right) \varepsilon^{2}-\frac{(\varepsilon, \psi)^{2}}{c_{0}} .
$$

Collecting (2-7) and (C-1), on the one hand, and (4-2) on the other hand, we compute

$$
\left|\int\left(Q^{2}-P_{B_{1}}^{2}\right) \varepsilon^{2}\right| \leq\left\|y^{2}\left(Q^{2}-P_{B_{1}}^{2}\right)\right\|_{L^{\infty}}\|\nabla \varepsilon\|_{L^{2}}^{2} \lesssim b\|\nabla \varepsilon\|_{L^{2}}^{2} \quad \text { and } \quad|(\varepsilon, \psi)|^{2} \lesssim b^{2}|\log b| .
$$

The nonlinear term is easily estimated by the Sobolev inequality:

$$
\int\left|\left(3 P_{B_{1}}+\varepsilon\right) \varepsilon^{3}\right| \leq\left\|y P_{B_{1}}\right\|_{L^{\infty}}\|y \varepsilon\|_{L^{\infty}}\|\nabla \varepsilon\|_{L^{2}}^{2} \lesssim \sqrt{b_{0}}\|\nabla \varepsilon\|_{L^{2}}^{2} .
$$

Injecting (4-45), (4-47), (4-46), (4-49), (4-48), (4-50) into (4-44) now yields (4-40).

Step 2: Lower bound on $b$. We now turn to the proof of (4-41). First observe from the bootstrap estimate (3-32) that

$$
\left|b_{s}\right| \leq \sqrt{K(M)} \frac{b^{2}}{|\log b|} \leq \frac{1-\alpha}{10} b^{2} .
$$

This implies

$$
\frac{d}{d s}\left(\frac{b^{4}}{(\log b)^{2} \lambda^{2(1-\alpha)}}\right)=\frac{4 b^{3}}{\lambda^{2(1-\alpha)}(\log b)^{2}}\left[b_{s}\left(1-\frac{1}{2 \log b}\right)+\frac{1-\alpha}{2} b^{2}\right]>0
$$

and (4-41) follows. 
Step 3: Improved $\dot{H}^{2}$ bound. We now turn to the proof of (4-43). We integrate (4-16) in time and conclude from (4-1) and (4-15) that

$$
\begin{aligned}
|\mathscr{E}(t)| \lesssim\left(\frac{\lambda(t)}{\lambda(0)}\right)^{2(1-\alpha)} & |\mathscr{E}(0)| \\
& +(K(M))^{1 / 2}\left[\frac{b^{4}(t)}{|\log b(t)|^{2}}+[\lambda(t)]^{2(1-\alpha)} \int_{0}^{t} \frac{b(\tau)}{[\lambda(\tau)]^{3-2 \alpha}} \frac{b^{4}(\tau)}{|\log b(\tau)|^{2}} d \tau\right] .
\end{aligned}
$$

We then derive from (4-51) that

$\int_{0}^{t} \frac{b(\tau)}{[\lambda(\tau)]^{3-2 \alpha}} \frac{b^{4}(\tau)}{|\log b(\tau)|^{2}} d \tau$

$$
\begin{aligned}
& =-\int_{0}^{t} \frac{\lambda_{t}}{\lambda^{3-2 \alpha}} \frac{b^{4}}{|\log b|^{2}} d \tau \\
& \leq \frac{1}{2(1-\alpha)} \frac{b^{4}(t)}{\lambda^{2(1-\alpha)}(t)|\log b(t)|^{2}}-\frac{1}{2(1-\alpha)} \int_{0}^{t} \frac{b_{s}}{\lambda^{3-2 \alpha}} \frac{b^{3}}{|\log b|^{2}}\left[1-\frac{2}{|\log b|^{2}}\right] \\
& \lesssim \frac{b^{4}(t)}{\lambda^{2(1-\alpha)}(t)|\log b(t)|^{2}}+\sqrt{K(M)} \int_{0}^{t} \frac{b(\tau)}{[\lambda(\tau)]^{3-2 \alpha}} \frac{b^{4}(\tau)}{|\log b(\tau)|^{2}} \frac{1}{|\log b(\tau)|} d \tau,
\end{aligned}
$$

and hence obtain the bound

$$
\lambda^{2(1-\alpha)}(t) \int_{0}^{t} \frac{b(\tau)}{[\lambda(\tau)]^{3-2 \alpha}} \frac{b^{4}(\tau)}{|\log b(\tau)|^{2}} d \tau \lesssim \frac{b^{4}(t)}{|\log b(t)|^{2}}
$$

Injecting this into (4-52) and using the initial bounds (A-12), (A-17) and the monotonicity (4-41) yields

$$
\mathscr{E}(t) \lesssim\left(\frac{\lambda(t)}{\lambda(0)}\right)^{2(1-\alpha)} \frac{b^{4}(0)}{|\log b(0)|^{2}}+(K(M))^{1 / 2} \frac{b^{4}(t)}{|\log b(t)|^{2}} \lesssim \sqrt{K(M)} \frac{b^{4}(t)}{|\log b(t)|^{2}}
$$

and (4-43) follows. The bound (4-42) now follows from Lemma 4.4 and (4-53). This concludes the proof of Lemma 4.9.

Dynamic of the unstable mode. We now focus onto the dynamic of the unstable mode. We recall the decomposition

$$
Y(t)=\mid \begin{aligned}
& (\varepsilon, \psi) \\
& \left(\partial_{s} \varepsilon, \psi\right)
\end{aligned}=\tilde{a}_{+}(t) V_{+}+\tilde{a}_{-}(t) V_{-},
$$

and the variables given by (3-22),

$$
\kappa_{+}(s)=\tilde{a}_{+}(s)+\frac{b_{s}}{2 \sqrt{\zeta}}\left(\partial_{b} P_{B_{1}}, \psi\right), \quad \kappa_{-}(s)=\tilde{a}_{-}(s)-\frac{b_{s}}{2 \sqrt{\zeta}}\left(\partial_{b} P_{B_{1}}, \psi\right) .
$$

Lemma 4.10 (control of the unstable mode). For all $t \in\left[0, T_{1}\left(a_{+}\right)\right]$we have

$$
\left|\kappa_{-}(t)\right| \leq \frac{1}{2}(K(M))^{1 / 8} \frac{b^{2}}{|\log b|},
$$

and $\kappa_{+}$is strictly outgoing,

$$
\left|\frac{d \kappa_{+}}{d s}-\sqrt{\zeta} \kappa_{+}\right| \leq \sqrt{b} \frac{b^{2}}{|\log b|}
$$


Proof. We compute the equation satisfied by the unstable direction $(\varepsilon, \psi)$ by taking the inner product of (3-11) with the well localized direction $\psi$ and get

$$
\frac{d^{2}}{d s^{2}}(\varepsilon, \psi)-\zeta(\varepsilon, \psi)=E(\varepsilon)-\left(\partial_{s}^{2} P_{B_{1}}, \psi\right)
$$

with

$$
\begin{aligned}
E(\varepsilon)=-\left(\Psi_{B_{1}}, \psi\right)-b_{s}( & \left.\Lambda P_{B_{1}}, \psi\right)-b\left(\partial_{s} P_{B_{1}}+2 \Lambda \partial_{s} P_{B_{1}}, \psi\right)-b\left(\partial_{s} \varepsilon+2 \Lambda \partial_{s} \varepsilon, \psi\right) \\
& -b_{s}(\Lambda \varepsilon, \psi)+(N(\varepsilon), \psi)+b^{2}(\Lambda \varepsilon, D \psi)+\left(\left(f^{\prime}\left(P_{B_{1}}\right)-f^{\prime}(Q)\right) \varepsilon, \psi\right) .
\end{aligned}
$$

Simple algebraic manipulations using (4-54) and (3-22) and the initial condition yield the equivalent system

$$
\frac{d}{d s} \kappa_{+}=\sqrt{\zeta} \kappa_{+}(s)+\frac{E_{+}(s)}{2 \sqrt{\zeta}}, \quad \frac{d}{d s} \kappa_{-}=-\sqrt{\zeta} \kappa_{-}(s)-\frac{E_{-}(s)}{2 \sqrt{\zeta}} \kappa_{-}(0),
$$

with

$$
E_{+}(s)=E(s)-\frac{b_{s}}{2}\left(\partial_{b} P_{B_{1}}, \psi\right), \quad E_{-}(s)=E(s)+\frac{b_{s}}{2}\left(\partial_{b} P_{B_{1}}, \psi\right) .
$$

We now have from the explicit formula (4-58) and (4-60), the exponential localization of $\psi$, the orthogonality

$$
(\psi, \Lambda Q)=0
$$

the estimates of Proposition 2.1 and the bootstrap estimate (3-32) the bound

$$
\frac{1}{\sqrt{\zeta}}\left|E_{ \pm}\right| \lesssim|b|\left(\left|b_{s}\right|+\sqrt{|\mathscr{E}|}+\sqrt{K(M)} \frac{b^{2}}{|\log b|}\right) \leq \sqrt{b} \frac{b^{2}}{|\log b|},
$$

which together with (4-59) yields (4-56). Let then

$$
\mathscr{G}=\kappa_{-}^{2} \frac{|\log b|^{2}}{b^{4}}
$$

then from (4-59), (4-61), (3-32), we estimate that

$$
\begin{aligned}
\frac{d \mathscr{G}}{d s} & =2 \kappa_{-} \frac{d \kappa_{-}}{d s} \frac{|\log b|^{2}}{b^{4}}+\kappa_{-}^{2} b_{s}\left[-\frac{4|\log b|^{2}}{b^{5}}+\frac{2 \log b}{b^{5}}\right] \\
& =2 \frac{|\log b|^{2}}{b^{4}}\left[\kappa_{-}\left(-\sqrt{\zeta} \kappa_{-}-\frac{E_{-}}{\sqrt{\zeta}}\right)\right]+\kappa_{-}^{2} \frac{|\log b|^{2}}{b^{4}} O\left(\frac{\left|b_{s}\right|}{b}\right) \\
& \leq-\frac{\sqrt{\zeta}}{2} \frac{|\log b|^{2}}{b^{4}} \kappa_{-}^{2}+\frac{|\log b|^{2}}{b^{4}} \kappa_{-} \sqrt{b} \frac{b^{2}}{|\log b|} \lesssim-\frac{\sqrt{\zeta}}{2} \varphi_{+1} .
\end{aligned}
$$

We integrate this in time and get

$$
\mathscr{G}(s) \leq \mathscr{G}(0) e^{-\frac{\sqrt{\zeta}}{2} s}+\int_{0}^{s} e^{-\frac{(s-\sigma)}{2} \sqrt{\zeta}} d \sigma \lesssim 1
$$

where we used the initial inequality (A-18) yielding that $\mathscr{G}(0) \lesssim 1$. This concludes the proof of (4-55) and of Lemma 4.10. 
Derivation of the sharp law for $\boldsymbol{b}$. We now turn to the derivation of the sharp law for $b$, which will yield the monotonicity statement on $b$ needed to obtain the smallness bootstrap estimate (3-31), and will eventually lead to the derivation of the sharp blow-up speed (1-10).

Lemma 4.11 (sharp law for $b$ ). Let

$$
\begin{aligned}
\tilde{P}_{B_{0}} & =\chi_{B_{0} / 4} Q \\
G(b) & =b\left|\Lambda \tilde{P}_{B_{0}}\right|_{L^{2}}^{2}+\int_{0}^{b} \tilde{b}\left(\partial_{b} \tilde{P}_{B_{0}}, \Lambda \tilde{P}_{B_{0}}\right) d \tilde{b}, \\
\mathscr{S}(s) & =\left(\partial_{s} \varepsilon, \Lambda \tilde{P}_{B_{0}}\right)+b\left(\varepsilon+2 \Lambda \varepsilon, \Lambda \tilde{P}_{B_{0}}\right)+b_{s}\left(\partial_{b} \tilde{P}_{B_{0}}, \Lambda \tilde{P}_{B_{0}}\right)-b_{s}\left(\partial_{b}\left(P_{B_{1}}-\tilde{P}_{B_{0}}\right), \Lambda \tilde{P}_{B_{0}}\right) .
\end{aligned}
$$

Then

$$
\begin{aligned}
& G(b)=64 b|\log b|+O(b), \quad|\mathscr{I}| \lesssim K(M) b, \\
& \left|\frac{d}{d s}\{G(b)+\mathscr{I}(s)\}+32 b^{2}\right| \lesssim K(M) \frac{b^{2}}{\sqrt{|\log b|}} .
\end{aligned}
$$

Remark 4.12. Observe that (4-65) and (4-66) essentially yield a pointwise differential equation

$$
b_{s} \sim-\frac{b^{2}}{2|\log b|},
$$

which will allow us to derive the sharp scaling law via the relationship $-\lambda_{s} / \lambda=b$.

Proof of Lemma 4.11. The proof is inspired by the one in [Raphaël and Rodnianski 2012]. We multiply (3-11) by $\Lambda \tilde{P}_{B_{0}}$ and compute

$$
\begin{aligned}
\left(b_{s} \Lambda P_{B_{1}}\right. & \left.+b\left(\partial_{s} P_{B_{1}}+2 \Lambda \partial_{s} P_{B_{1}}\right)+\partial_{s}^{2} P_{B_{1}}, \Lambda \tilde{P}_{B_{0}}\right) \\
& =-\left(\Psi_{B_{1}}, \Lambda \tilde{P}_{B_{0}}\right)-\left(H_{B_{1}} \varepsilon, \Lambda \tilde{P}_{B_{0}}\right)-\left(\partial_{s}^{2} \varepsilon+b\left(\partial_{s} \varepsilon+2 \Lambda \partial_{s} \varepsilon\right)+b_{s} \Lambda \varepsilon, \Lambda \tilde{P}_{B_{0}}\right)+\left(N(\varepsilon), \Lambda \tilde{P}_{B_{0}}\right)
\end{aligned}
$$

We further rewrite this as

$$
\begin{aligned}
& \left(b_{s} \Lambda \tilde{P}_{B_{0}}+b\left(\partial_{s} \tilde{P}_{B_{0}}+2 \Lambda \partial_{s} \tilde{P}_{B_{0}}\right)+\partial_{s}^{2} \tilde{P}_{B_{0}}, \Lambda \tilde{P}_{B_{0}}\right) \\
& =-\left(\Psi_{B_{1}}, \Lambda \tilde{P}_{B_{0}}\right)-\left(b_{s} \Lambda\left(P_{B_{1}}-\tilde{P}_{B_{0}}\right)+b\left(\partial_{s}\left(P_{B_{1}}-\tilde{P}_{B_{0}}\right)+2 \Lambda \partial_{s}\left(P_{B_{1}}-\tilde{P}_{B_{0}}\right)\right)+\partial_{s}^{2}\left(P_{B_{1}}-\tilde{P}_{B_{0}}\right), \Lambda \tilde{P}_{B_{0}}\right) \\
& -\left(H_{B_{1}} \varepsilon, \Lambda \tilde{P}_{B_{0}}\right)-\left(\partial_{s}^{2} \varepsilon+b\left(\partial_{s} \varepsilon+2 \Lambda \partial_{s} \varepsilon\right)+b_{s} \Lambda \varepsilon, \Lambda \tilde{P}_{B_{0}}\right)+\left(N(\varepsilon), \Lambda \tilde{P}_{B_{0}}\right) .
\end{aligned}
$$

We now estimate all terms in this identity.

Step 1: $b$ terms. An integration by parts in time allows us to rewrite the left-hand side of (4-67) as

$$
\left(b_{s} \Lambda \tilde{P}_{B_{0}}+b\left(\partial_{s} \tilde{P}_{B_{0}}+2 \Lambda \partial_{s} \tilde{P}_{B_{0}}\right)+\partial_{s}^{2} \tilde{P}_{B_{0}}, \Lambda \tilde{P}_{B_{0}}\right)=\frac{d}{d s}\left[G(b)+b_{s}\left(\partial_{b} \tilde{P}_{B_{0}}, \Lambda \tilde{P}_{B_{0}}\right)\right]+\left|b_{s}\right|^{2}\left\|\partial_{b} \tilde{P}_{B_{0}}\right\|_{L^{2}}^{2}
$$

with $G$ given by (4-63). Observe from (3-32) the bound

$$
\left|b_{s}\right|^{2}\left\|\partial_{b} \tilde{P}_{B_{0}}\right\|_{L^{2}}^{2} \lesssim \frac{\left|b_{s}\right|^{2}}{b^{2}} \lesssim(K(M))^{2} \frac{b^{2}}{|\log b|^{2}} \lesssim \frac{b^{2}}{\sqrt{|\log b|}} .
$$


We now turn to the key step in the derivation of the sharp $b$ law which corresponds to the following outgoing flux computation: ${ }^{8}$

$$
\left(\Psi_{B_{1}}, \Lambda \tilde{P}_{B_{0}}\right)=32 b^{2}\left(1+O\left(\frac{1}{|\log b|}\right)\right) \text { as } b \rightarrow 0 .
$$

Indeed, we first estimate from (2-9) that

$$
\left|\left(\Psi_{B_{1}}-c_{b} b^{2} \chi_{B_{0} / 4} \Lambda Q, \Lambda \tilde{P}_{B_{0}}\right)\right| \lesssim b^{4} \int_{y \leq B_{0} / 2}\left[\frac{1+|\log (b y)|}{|\log b|\left(1+y^{2}\right)}+\frac{1+|\log (1+y)|}{\left(1+y^{2}\right)^{2}}\right] \lesssim \frac{b^{2}}{|\log b|} .
$$

The remainder term is computed from (2-10) and the explicit formula for $Q(1-3)$,

$$
\left(c_{b} b^{2} \chi_{B_{0} / 4} \Lambda Q, \Lambda \tilde{P}_{B_{0}}\right)=\frac{b^{2}}{2|\log b|}\left(1+O\left(\frac{1}{|\log b|}\right)\right)\left[\int_{y \leq 1 / 2 b}(\Lambda Q)^{2}+O(1)\right]=32 b^{2}\left(1+O\left(\frac{1}{|\log b|}\right)\right),
$$

and (4-69) follows.

We now estimate the lower-order terms in $b$ that correspond to the second line of (4-67). One term is reintegrated by parts in time,

$$
-\left(\partial_{s}^{2}\left(P_{B_{1}}-\tilde{P}_{B_{0}}\right), \Lambda \tilde{P}_{B_{0}}\right)=-\frac{d}{d s}\left\{b_{s}\left(\partial_{b}\left(P_{B_{1}}-\tilde{P}_{B_{0}}\right), \Lambda \tilde{P}_{B_{0}}\right)\right\}+b_{s}^{2}\left(\partial_{b}\left(P_{B_{1}}-\tilde{P}_{B_{0}}\right), \partial_{b} \Lambda \tilde{P}_{B_{0}}\right) .
$$

The remaining terms are estimated in brute force using (2-8) and (3-32), which yield

$$
\begin{aligned}
\left|\left(b_{s} \Lambda\left(P_{B_{1}}-\tilde{P}_{B_{0}}\right)+b\left(\partial_{s}\left(P_{B_{1}}-\tilde{P}_{B_{0}}\right)+2 \Lambda \partial_{s}\left(P_{B_{1}}-\tilde{P}_{B_{0}}\right)\right), \Lambda \tilde{P}_{B_{0}}\right)\right|+b_{s}^{2} \mid & \left(\partial_{b}\left(P_{B_{1}}-\tilde{P}_{B_{0}}\right), \partial_{b} \Lambda \tilde{P}_{B_{0}}\right) \mid \\
& \lesssim\left|b_{s}\right|+\frac{\left|b_{s}\right|^{2}}{b^{2}} \lesssim K(M) \frac{b^{2}}{|\log b|} .
\end{aligned}
$$

Step 2: $\varepsilon$ terms. We are left with estimating the third line on the right-hand side of (4-67). We first treat the linear term from (4-1), (4-7) and (3-34) and get

$$
\left|\left(H_{B_{1}} \varepsilon, \Lambda \tilde{P}_{B_{0}}\right)\right| \lesssim\left|\left(H \varepsilon, \Lambda \tilde{P}_{B_{0}}\right)\right|+\int|\varepsilon|\left|P_{B_{1}}^{2}-Q^{2}\right|\left|\Lambda \tilde{P}_{B_{0}}\right|+b^{2}\left|\left(D \Lambda \varepsilon, \Lambda \tilde{P}_{B_{0}}\right)\right|
$$

On the one hand, (4-7) together with bootstrap estimates yields

$$
\int|\varepsilon|\left|P_{B_{1}}^{2}-Q^{2}\right|\left|\Lambda \tilde{P}_{B_{0}}\right| \lesssim b^{2} \int_{y \leq B_{0}} \frac{|\varepsilon|}{\left(1+y^{2}\right)^{2}} \leq b^{3 / 2}\left(\int \frac{|\varepsilon|^{2}}{(1+y)^{5}}\right)^{1 / 2} \lesssim \frac{b^{2}}{|\log (b)|}
$$

On the other hand, after integration by parts, we repeat the same arguments as before and apply (C-4).

\footnotetext{
${ }^{8}$ See again [Raphaël and Rodnianski 2012] for more details about the flux computation statement and its connection to the Pohozaev integration by parts formula.
} 
This yields

$$
\begin{aligned}
b^{2}\left|\left(D \Lambda \varepsilon, \Lambda \tilde{P}_{B_{0}}\right)\right| & \leq b^{2} \int_{y \leq B_{0}} \frac{|\varepsilon|}{\left(1+y^{4}\right)}+b^{2} \int_{B_{0} / 4 \leq y \leq B_{0} / 2} \frac{|\varepsilon|}{\left(1+y^{2}\right)}+b^{2} \int_{y \leq B_{0}}|\nabla \varepsilon| \frac{y}{1+y^{2}} \\
& \lesssim b^{3 / 2}\left(\int \frac{|\varepsilon|^{2}}{\left(1+y^{5}\right)}\right)^{1 / 2}+\left(\int_{B_{0} / 4 \leq y \leq B_{0} / 2} \frac{|\varepsilon|^{2}}{\left(1+y^{4}\right)}\right)^{1 / 2}+\left(\int_{y \leq B_{0}} \frac{|\nabla \varepsilon|^{2}}{1+y^{2}}\right)^{1 / 2} \\
& \lesssim \sqrt{|\log (b)|}\left(c(M)|\mathscr{E}|+\sqrt{K(M)} \frac{b^{4}}{|\log b|^{2}}\right)^{1 / 2} \\
& \lesssim \sqrt{K(M)} \frac{b^{2}}{\sqrt{|\log (b)|}} .
\end{aligned}
$$

Finally,

$$
\begin{aligned}
\left|\left(H \varepsilon, \Lambda \tilde{P}_{B_{0}}\right)\right| & \lesssim\|H \varepsilon\|_{L^{2}} \sqrt{|\log b|}+\sqrt{K(M)} \frac{b^{2}}{\sqrt{\log (b)}} \\
& \lesssim \sqrt{|\log b|} \sqrt{|\mathscr{E}|+\sqrt{K(M)} b^{4} /|\log b|^{2}} \lesssim \sqrt{K(M)} \frac{b^{2}}{\sqrt{|\log b|}} .
\end{aligned}
$$

We further integrate by parts in time to obtain

$$
\begin{aligned}
\left(\partial_{s}^{2} \varepsilon+b\left(\partial_{s} \varepsilon+2 \Lambda \partial_{s} \varepsilon\right)\right. & \left.+b_{s} \Lambda \varepsilon, \Lambda \tilde{P}_{B_{0}}\right) \\
& =\frac{d}{d s}\left[\left(\partial_{s} \varepsilon, \Lambda \tilde{P}_{B_{0}}\right)+b\left(\varepsilon+2 \Lambda \varepsilon, \Lambda \tilde{P}_{B_{0}}\right)\right]-b_{s}\left[\left(\partial_{s} \varepsilon+b \Lambda \varepsilon, \Lambda \partial_{b} \tilde{P}_{B_{0}}\right)+\left(\varepsilon, \Phi_{b}\right)\right]
\end{aligned}
$$

with

$$
\Phi_{b}=-\Lambda \tilde{P}_{B_{0}}-\Lambda^{2} \tilde{P}_{B_{0}}-b \Lambda \partial_{b} \tilde{P}_{B_{0}}-b \Lambda^{2} \partial_{b} \tilde{P}_{B_{0}}
$$

We thus estimate from (4-1), (4-5), (4-7), (3-32) and (3-34) that

$$
\begin{aligned}
\left|b_{s}\right|\left|\left(\partial_{s} \varepsilon+b \Lambda \varepsilon, \Lambda \partial_{b} \tilde{P}_{B_{0}}\right)+\left(\varepsilon, \Phi_{b}\right)\right| & \lesssim\left|b_{s}\right|\left[\int_{B_{0} / 4 \leq y \leq B_{0}} \frac{|\eta|}{y}+\int_{y \leq B_{0}} \frac{|\varepsilon|}{1+y^{2}}\right] \\
& \lesssim \frac{\left|b_{s}\right||\log b|}{b^{2}} C(M) \sqrt{|\mathscr{E}|+\sqrt{K(M)} b^{4} /|\log b|^{2}} \lesssim K(M) \frac{b^{2}}{\sqrt{|\log b|}} .
\end{aligned}
$$

The nonlinear term is estimated as before. Indeed, we have

$$
\begin{aligned}
\left|\left(N(\varepsilon), \Lambda \tilde{P}_{B_{0}}\right)\right| & \lesssim \int\left(\left|P_{B_{1}}\right|+|\varepsilon|\right) \varepsilon^{2}\left|\Lambda \tilde{P}_{B_{0}}\right| \\
& \lesssim \frac{1}{b^{2}}\left\|y\left(\left|P_{B_{1}}\right|+|\varepsilon|\right)\right\|_{L^{\infty}}\left\|\left(1+y^{2}\right) \Lambda \tilde{P}_{B_{0}}\right\|_{L^{\infty}} \int_{0}^{B_{0}} \frac{|\varepsilon|^{2}}{y\left(1+y^{4}\right)} \\
& \lesssim \frac{C(M)}{b^{2}}\left[\mathscr{E}+K(M) \frac{b^{4}}{|\log b|^{2}}\right] \lesssim K(M) \frac{b^{2}}{\sqrt{|\log b|}}
\end{aligned}
$$

Step 3: Control of $G(b)$ and $\Phi$. Injecting the estimates of Steps 1 and 2 into (4-67) yields (4-66). It remains to prove (4-65). The estimate for $G(b)$ is a straightforward consequence of the choice (4-62) and 
the explicit formula (1-3). It remains to control $\mathscr{I}$. We integrate by parts in space in (4-64) and get

$$
\mathscr{I}(s)=\left(\partial_{s} \varepsilon+b \Lambda \varepsilon, \Lambda \tilde{P}_{B_{0}}\right)-b\left(\varepsilon, \Lambda \tilde{P}_{B_{0}}+\Lambda^{2} \tilde{P}_{B_{0}}\right)+b_{s}\left(\partial_{b} \tilde{P}_{B_{0}}, \Lambda \tilde{P}_{B_{0}}\right)-b_{s}\left(\partial_{b}\left(P_{B_{1}}-\tilde{P}_{B_{0}}\right), \Lambda \tilde{P}_{B_{0}}\right) .
$$

The $b$ terms are estimated as in Step 1,

$$
\left|b_{s}\right|\left|\left(\partial_{b} \tilde{P}_{B_{0}}, \Lambda \tilde{P}_{B_{0}}\right)-\left(\partial_{b}\left(P_{B_{1}}-\tilde{P}_{B_{0}}\right), \Lambda \tilde{P}_{B_{0}}\right)\right| \lesssim \frac{\left|b_{s}\right|}{b} \lesssim b
$$

The linear term is estimated using (4-1), (4-5), (4-7), (3-32) and (3-34),

$$
\begin{aligned}
\left|\left(\partial_{s} \varepsilon+b \Lambda \varepsilon, \Lambda \tilde{P}_{B_{0}}\right)-b\left(\varepsilon, \Lambda \tilde{P}_{B_{0}}+\Lambda^{2} \tilde{P}_{B_{0}}\right)\right| & \\
& \lesssim \int_{y \leq B_{0}} \frac{|\eta|}{y^{2}}+b \int_{y \leq B_{0}} \frac{|\varepsilon|}{y^{2}} \lesssim \frac{1}{b}\left(\int \frac{|\eta|^{2}}{y^{2}}\right)^{1 / 2}+\frac{|\log b|}{b^{2}}\left(\int_{y \leq B_{0}} \frac{|\varepsilon|^{2}}{y^{4}\left(1+|\log y|^{2}\right)}\right)^{1 / 2} \lesssim K(M) b,
\end{aligned}
$$

and (4-65) is proved. This concludes the proof of Lemma 4.11.

\section{Sharp description of the singularity formation}

We are now in position to conclude the proofs of Proposition 3.5 and Theorem 1.1 as simple consequences of the a priori bounds obtained in the previous section. The proofs rely on a topological argument that finishes the bootstrap argument, and then the sharp description of the blow-up dynamic is a consequence of the a priori bounds obtained on the solution and in particular the modulation equation (4-66).

Proof of Proposition 3.5. We argue by contradiction and assume that for all

$$
a_{+} \in\left[-\frac{b_{0}^{2}}{\left|\log b_{0}\right|}, \frac{b_{0}^{2}}{\left|\log b_{0}\right|}\right], \quad T_{1}\left(a_{+}\right)<T\left(a_{+}\right) .
$$

In view of what Lemma 4.9 says about the bootstrap regime and the improved bounds of Lemmas 4.9 and 4.10, a simple continuity argument ensures that $T_{1}\left(a_{+}\right)$is attained at the first time $t$ where

$$
\left|\kappa_{+}(t)\right|=\frac{|b(t)|^{2}}{2|\log (b(t))|} .
$$

The fundamental fact used now is the outgoing behavior (4-56), which together with (5-1), ensures that

$$
\left|\frac{d \kappa_{+}}{d t}\left(T_{1}\left(a_{+}\right)\right)\right|>0
$$

Thus from a standard argument, ${ }^{9}$ the map

$$
\left[-\frac{b_{0}^{2}}{\left|\log b_{0}\right|}, \frac{b_{0}^{2}}{\left|\log b_{0}\right|}\right] \rightarrow \mathbb{R} *_{+}, \quad a_{+} \mapsto T_{1}\left(a_{+}\right),
$$

is continuous. We may thus consider the continuous map

$$
\Phi:\left[-\frac{b_{0}^{2}}{\left|\log b_{0}\right|}, \frac{b_{0}^{2}}{\left|\log b_{0}\right|}\right] \rightarrow \mathbb{R}, \quad a_{+} \rightarrow \kappa_{+}\left(T_{1}\left(a_{+}\right)\right) \frac{2\left|\log b\left(T_{1}\left(a_{+}\right)\right)\right|}{b^{2}\left(T_{1}\left(a_{+}\right)\right)}
$$

\footnotetext{
${ }^{9}$ See [Cote et al. 2009, Lemma 6] for a complete exposition.
} 
On the one hand, (5-1) implies

$$
\Phi\left(\left[-\frac{b_{0}^{2}}{\left|\log b_{0}\right|}, \frac{b_{0}^{2}}{\left|\log b_{0}\right|}\right]\right) \subset\{-1,1\} .
$$

On the other hand, the outgoing behavior (4-56) together with the initialization $\kappa_{+}(0)=a_{+}$ensure that

$$
\Phi\left(-\frac{b_{0}^{2}}{\left|\log b_{0}\right|}\right)=-1 \quad \text { and } \Phi\left(\frac{b_{0}^{2}}{\left|\log b_{0}\right|}\right)=1
$$

and a contradiction follows. ${ }^{10}$ This concludes the proof of Proposition 3.5.

Proof of Theorem 1.1.

Step 1: Finite time blow-up and derivation of the blow-up speed. Choose from Proposition 3.5 initial data with $T_{1}\left(a_{+}\right)=T\left(a_{+}\right)$. We first claim that $u$ blows up in finite time,

$$
T=T\left(a_{+}\right)<+\infty .
$$

Indeed, from (4-41),

$$
\lambda^{2(1-\alpha)} \lesssim b^{3} \text { and thus } \quad \lambda^{2 / 3} \lesssim \lambda^{2(1-\alpha) / 3} \lesssim b=-\lambda_{t} .
$$

Integrating this differential inequality yields

$$
t \lesssim \lambda^{1 / 3}(0)-\lambda^{1 / 3}(t) \lesssim 1
$$

and (5-2) follows. The $\left(\dot{H}^{1} \cap \dot{H}^{2}\right) \times\left(L^{2} \cap \dot{H}^{1}\right)$ bounds (3-33) and (3-34) on $\left(\varepsilon, \partial_{t} \varepsilon\right)$, and hence on $\left(u, \partial_{t} u\right)$ in the bootstrap regime, and standard $H^{2}$ local well posedness theory ensures that blow-up corresponds to

$$
\lambda(t) \rightarrow 0 \quad \text { as } t \rightarrow T\left(a_{+}\right) .
$$

We now derive the blow-up speed by reintegrating the ODE (4-66) and briefly sketch the proof which follows as in [Raphaël and Rodnianski 2012].

First recall the standard scaling lower bound

$$
\lambda(t) \leq C\left(u_{0}\right)(T-t),
$$

which implies that the rescaled time is global,

$$
s(t)=\int_{0}^{t} \frac{d \tau}{\lambda(\tau)} \rightarrow+\infty \quad \text { as } t \rightarrow T .
$$

Let

$$
\mathscr{g}=G+\mathscr{g}
$$

so that from (4-65) we get

$$
\mathscr{F}=64 b|\log b|\left(1+O\left(\frac{1}{|\log b|}\right)\right) \text { and } \quad b=\frac{\mathscr{E}}{64|\log \mathscr{F}|}\left(1+O\left(\frac{1}{\sqrt{|\log \mathscr{F}|}}\right)\right),
$$

\footnotetext{
${ }^{10}$ This topological argument is the one-dimensional version of Brouwer's fixed-point argument used in [Cote et al. 2009].
} 
and $\mathscr{I}$ satisfies from (4-66) the ODE

$$
\mathscr{F}_{s}+\frac{\mathscr{g}^{2}}{128|\log \mathscr{F}|^{2}}\left(1+O\left(\frac{1}{\sqrt{|\log \mathscr{F}|}}\right)\right)=0 .
$$

We multiply the above by $|\log \mathscr{F}|^{2} / \mathscr{F}^{2}$, integrate in time and obtain to leading order that

$$
\mathscr{g}=\frac{128(\log s)^{2}}{s}\left(1+O\left(\frac{1}{\sqrt{|\log s|}}\right)\right) \text { that is, }-\frac{\lambda_{s}}{\lambda}=b=\frac{2 \log s}{s}\left(1+O\left(\frac{1}{\sqrt{|\log s|}}\right)\right),
$$

where we used (5-3). Integrating this once more in time yields

$$
-\log \lambda=(\log s)^{2}\left(1+O\left(\frac{1}{\sqrt{|\log s|}}\right)\right)
$$

and thus

$$
b=-\lambda_{t}=\exp \left(-\sqrt{|\log \lambda|}\left(1+O\left(\frac{1}{|\log \lambda|^{1 / 4}}\right)\right)\right) .
$$

Integrating this from $t$ to $T$ where $\lambda(T)=0$ yields the asymptotic

$$
\lambda(t)=(T-t) \exp \left(-\sqrt{|\log \lambda(t)|}\left(1+O\left(\frac{1}{|\log \lambda(t)|^{1 / 4}}\right)\right)\right),
$$

which yields (1-10).

Step 2: Energy quantization. It remains to prove (1-9), which can be derived exactly as in [Raphaël and Rodnianski 2012]; this is left to the reader. This concludes the proof of Theorem 1.1.

\section{Appendix A: Modulation theory}

This appendix is devoted to the proof of Lemmas 3.1 and 3.3. The arguments are standard in the framework of modulation theory and we briefly sketch the main computations.

Proof of Lemma 3.1. First note that the bounds

$$
\left\|\nabla\left(P_{B_{1}}-Q\right)\right\|_{L^{2}}+b\left\|\Lambda P_{B_{1}}-b\left(1-\chi_{B_{1}}\right) \Lambda Q\right\|_{L^{2}} \lesssim b|\log b|
$$

ensure that our initial data are of the form

$$
u_{0}=Q+\tilde{\eta}_{0}, \quad u_{1}=\tilde{\eta}_{1},
$$

for a small excess of energy in the sense that

$$
\left\|\nabla \tilde{\eta}_{0}, \tilde{\eta}_{1}\right\|_{L^{2} \times L^{2}} \lesssim b_{0}\left|\log b_{0}\right|, \quad\left\|\nabla^{2} \tilde{\eta}_{0}, \nabla \tilde{\eta}_{1}\right\|_{L^{2} \times L^{2}} \lesssim b_{0} .
$$

Hence the continuity of the flow associated to (1-1) ensures the existence of a time $T_{0}>0$ (uniform in $\left.\tilde{\eta}_{0}, \tilde{\eta}_{1}\right)$ for which the solution $u$ to (1-1) with initial data $\left(u_{0}, u_{1}\right)$ satisfies on $\left[0, T_{0}\right]$ that

$$
\sup _{\left[0, T_{0}\right]}\left\|\nabla(u-Q), \partial_{t} u\right\|_{L^{2} \times L^{2}} \lesssim b_{0}\left|\log b_{0}\right| .
$$


Step 1: Modulation near $Q$. The nondegeneracy $(\Lambda Q, \Phi) \neq 0$ ensures ${ }^{11}$ that $u$ admits on $\left[0, T_{0}\right]$ a decomposition

$$
u(t)=(Q+\tilde{\varepsilon}(t))_{\lambda(t)},
$$

with

$$
\left(\tilde{\varepsilon}(t), \chi_{M} \Phi\right)=0 .
$$

Moreover, $\lambda \in \mathscr{C}^{2}\left(\left[0, T_{0}\right] ; \mathbb{R}_{+}^{*}\right)$, and noting that $\tilde{\eta}_{0}$ satisfies

$$
\left|\left(\tilde{\eta}_{0}, \chi_{M} \Phi\right)\right| \lesssim \frac{b_{0}^{2}}{\left|\log b_{0}\right|},
$$

we obtain the bound

$$
|\lambda(0)-1| \lesssim \frac{b_{0}^{2}}{\left|\log b_{0}\right|}
$$

We then let $b(t)=-\lambda_{t}(t)$ on $\left[0, T_{0}\right]$.

Step 2: Positivity of $b$. Straightforward computations yield

$$
\partial_{t} \tilde{\varepsilon}(t)=\left(\partial_{t} u-\frac{b(t)}{\lambda(t)} \Lambda u\right)_{1 / \lambda(t)} .
$$

Taking the scalar product with $\chi_{M} \Phi$, we obtain at the initial time

$$
b(0)=\lambda(0) \frac{\left(\left(u_{1}\right)_{1 / \lambda(0)}, \chi_{M} \Phi\right)}{\left(\left(\Lambda u_{0}\right)_{1 / \lambda(0)}, \chi_{M} \Phi\right)}
$$

where (2-5) together with (A-5) imply

$$
\begin{aligned}
\left(\left(u_{1}\right)_{1 / \lambda(0)}, \chi_{M} \Phi\right) & =b_{0}\left(\Lambda Q, \chi_{M} \Phi\right)+O\left(\frac{b_{0}^{2}}{\left|\log \left(b_{0}\right)\right|}\right), \\
\left(\left(\Lambda u_{0}\right)_{1 / \lambda(0)}, \chi_{M} \Phi\right) & =\left(\Lambda Q, \chi_{M} \Phi\right)+O\left(b_{0}^{2}\left|\log \left(b_{0}\right)\right|\right) .
\end{aligned}
$$

This yields the positivity of $b(0)$ and the positivity of $b(t)$ for small time, together with

$$
b(t)=b_{0}+O\left(\frac{b_{0}^{2}}{\left|\log \left(b_{0}\right)\right|}\right) .
$$

As $b>0$, we may introduce the decomposition

$$
u(t)=(Q+\tilde{\varepsilon})_{\lambda(t)}=\left(P_{B_{1}(b(t))}+\varepsilon\right)_{\lambda(t)}, \quad \text { where } \varepsilon(t)=\tilde{\varepsilon}(t)-\left(P_{B_{1}(b(t))}-Q\right) .
$$

Observe from (2-4) and (A-4) that

$$
\forall t \in\left[0, T_{0}\right], \quad\left(\varepsilon(t), \chi_{M} \Phi\right)=0 .
$$

The uniqueness of such a decomposition is guaranteed by the (local) uniqueness of $(\lambda, \tilde{\varepsilon})$.

\footnotetext{
${ }^{11}$ This is a direct consequence of the implicit function theorem and the smoothness of the flow (1-1).
} 
Step 3: Smallness of $\varepsilon$. To complete the proof, we obtain smallness of $\varepsilon$ in $\dot{H}^{1}$ and $\dot{H}^{2}$. To this end, we note that

$$
\varepsilon(0)=\left(u_{0}\right)_{1 / \lambda(0)}-P_{B_{1}(b(0))}=\left[\left(P_{B_{1}\left(b_{0}\right)}\right)_{1 / \lambda(0)}-P_{B_{1}(b(0))}\right]+\left(\eta_{0}+d_{+} \psi\right)_{1 / \lambda(0)} .
$$

Simple computations based on the estimates of Proposition 2.1 yield the expected result,

$$
\|\nabla \varepsilon(0)\|_{L^{2}} \lesssim b_{0}\left|\log \left(b_{0}\right)\right| \quad \text { and } \quad\left\|\frac{\varepsilon(0)}{1+y^{4}}\right\|_{L^{2}}+\left\|\nabla^{2} \varepsilon(0)\right\|_{L^{2}} \lesssim \frac{b_{0}^{2}}{\left|\log \left(b_{0}\right)\right|} .
$$

This concludes the proof.

Proof of Lemma 3.3. The proof of this lemma is divided into two steps. First, given $\left(\eta_{0}, \eta_{1}, d_{+}\right)$satisfying the smallness condition (3-1) for small $b_{0}$, we prove that $b, b_{s}$ and $w$ satisfy (3-31)-(3-34). Then, we show that given $\left(b_{0}, \eta_{0}, \eta_{1}\right)$, we can apply the inverse mapping theorem to $d_{+} \mapsto \kappa_{+}(0)$ close to 0 . The arguments used are standard and we refer to [Cote et al. 2009] for a detailed proof in a similar setting.

Step 1: Smallness of initial modulation. Given $\left(\eta_{0}, \eta_{1}, d_{+}\right)$satisfying the smallness condition (3-1), we can apply Lemma 3.1. This yields $T_{0}$ and $b, \varepsilon, w$ such that (3-31) holds and

$$
\|\nabla w(t)\|_{L^{2}} \lesssim b_{0}\left|\log \left(b_{0}\right)\right|, \quad\left\|\nabla^{2} w(t)\right\|_{L^{2}} \lesssim \frac{b_{0}^{2}}{\left|\log \left(b_{0}\right)\right|^{2}} .
$$

We emphasize that Lemma 3.1 implies in particular that $b_{0} / 2<b(0)<2 b_{0}$ for sufficiently small $b_{0}$.

As before, we focus now on bounds satisfied initially. We first compute $b_{s}(0)$ using (1-1) and the orthogonality condition (A-11). Recalling that $\left(\partial_{b}^{k} P_{B_{1}}, \chi_{M} \Phi\right)=\left(\partial_{s}^{k-1} \varepsilon, \chi_{M} \Phi\right)=0$ for any integer $k$, we get, like for (4-10),

$$
\begin{aligned}
b_{s}\left[\left(\Lambda P_{B_{1}}, \chi_{M} \Phi\right)+2 b\left(\Lambda \partial_{b} P_{B_{1}},\right.\right. & \left.\left.\chi_{M} \Phi\right)+\left(\Lambda \varepsilon, \chi_{M} \Phi\right)\right] \\
& =-\left(\Psi_{B_{1}}, \chi_{M} \Phi\right)-\left(\varepsilon, H_{B_{1}}^{*}\left(\chi_{M} \Phi\right)\right)+b\left(\partial_{s} \varepsilon, \Lambda\left(\chi_{M} \Phi\right)\right)+\left(N(\varepsilon), \chi_{M} \Phi\right)
\end{aligned}
$$

where, denoting by $L H S$ and RHS the two sides at initial time, we compute, for $b_{0}$ small enough with respect to $M$ that

$$
|R H S| \leq C(M)\left(\frac{b_{0}^{2}}{\left|\log \left(b_{0}\right)\right|}+\left\|\partial_{s} \varepsilon\right\|_{L^{2}(y<M)}\right), \quad \frac{\left|b_{s}(0)\right|}{2}\left(\Lambda Q, \chi_{M} \Phi\right) \leq|L H S| .
$$

At the same time, after time-differentiation, we obtain

$$
\partial_{s} \varepsilon(0)=\lambda(0) \partial_{t} \varepsilon(0)=-b_{s}(0) \partial_{b} P_{B_{1}(b(0))}-b(0) \Lambda u_{0}+\lambda(0)\left(b_{0} \Lambda P_{B_{1}\left(b_{0}\right)}\right)_{1 / \lambda(0)} .
$$

Observe now from (2-8) that

$$
\left\|\partial_{b} P_{B_{1}\left(b_{0}\right)}\right\|_{L^{2}(y \leq 2 M)} \lesssim C(M) b_{0} \leq \sqrt{b_{0}},
$$

which together with (A-5), (A-9) and (3-1) yields

$$
\left\|\partial_{s} \varepsilon(0)\right\|_{L^{2}(y \leq 2 M)}=\lambda(0)\left\|\partial_{t} \varepsilon(0)\right\|_{L^{2}(y \leq 2 M)} \lesssim \frac{b_{0}^{2}}{\left|\log b_{0}\right|}+\left|b_{s}(0)\right| \sqrt{b_{0}},
$$


which together with (A-14) concludes the proof of the initial bound (3-26) on $b_{s}$.

Then we compute

$$
\partial_{t} w(0)=u_{1}-\left(\frac{b_{s}(0)}{\lambda(0)} \partial_{b} P_{B_{1}(b(0))}+\frac{b(0)}{\lambda(0)} \Lambda P_{B_{1}(b(0))}\right)_{\lambda(0)},
$$

so that, introducing (A-15) and previous estimates on $b(0)$, we get

$$
\left\|\partial_{t} w(0)+\frac{b(0)}{\lambda(0)}\left(\left(1-\chi_{B_{1}(b(0))}\right) \Lambda Q\right)_{\lambda(0)}\right\|_{L^{2}} \lesssim b_{0}\left|\ln \left(b_{0}\right)\right| \leq \sqrt{b_{0}},
$$

and

$$
\left\|\nabla \partial_{t} w(0)\right\|_{L^{2}} \lesssim \frac{b_{0}^{2}}{\left|\log b_{0}\right|}
$$

Together with (A-13), this yields (3-27) and (3-28).

Finally, straightforward computations yield

$$
\kappa_{-}=\frac{1}{2}(\varepsilon, \psi)-\frac{1}{\zeta}\left(\partial_{s} \varepsilon, \psi\right)-\frac{b_{s}}{2 \zeta}\left(\partial_{b} P_{B_{1}}, \psi\right)
$$

Consequently, we apply (3-28), noting that $w(t)=(\varepsilon(t))_{\lambda(t)}$, and (A-15) because of the exponential decay of $\psi$ to get

$$
\left|\kappa_{-}(0)\right| \lesssim \frac{b_{0}^{2}}{\left|\log b_{0}\right|}
$$

Step 2: Computation of $d_{+}$. We now claim from an explicit computation that given $a_{+}$, the initialization $\overline{(3-24)}$ can be reformulated in the form

$$
F\left(d_{+}\right)=a_{+}, \quad \text { with }\left.\frac{\partial F}{\partial d_{+}}\right|_{d_{+}=0}=\frac{1}{2}\|\psi\|_{L^{2}}^{2}+O\left(b_{0}\right),
$$

from which the implicit function theorem concludes the proof of Lemma 3.3.

Let us briefly justify (A-19). We want to study the mapping

$$
\mathscr{V} \rightarrow \mathbb{R}^{4}, \quad d_{+} \mapsto\left[b(t), b_{s}(t),(\varepsilon(0), \psi),\left(\partial_{s} \varepsilon(t), \psi\right)\right],
$$

where $\mathscr{V}$ is a neighborhood of 0 . To this end, it is necessary to study the dependencies of all initial parameters on $d_{+}$. For conciseness, we denote by $d$ differentiation with respect to $d_{+}$in what follows.

Computation of $(\boldsymbol{\lambda}(\mathbf{0}), \tilde{\varepsilon}(\mathbf{0}))$. As a first step in modulation theory, we proved that $(\lambda(0), \tilde{\varepsilon}(0))=\Phi\left(u_{0}\right)$, where $\Phi$ is a smooth mapping $\dot{H}^{1}\left(\mathbb{R}^{N}\right) \rightarrow \mathbb{R} \times \dot{H}^{1}\left(\mathbb{R}^{N}\right)$ defined on a neighborhood of $Q$. Due to the exponential decay of $\psi \in \mathscr{C}^{\infty}\left(\mathbb{R}^{N}\right)$ we thus have that $\lambda(0)$ is a smooth function of $d_{+}$with differential $d \lambda(0)=d \lambda \in \mathbb{R}$. We have the same result for $\varepsilon$ with differential $d \tilde{\varepsilon}(0)=d \tilde{\varepsilon} \in \dot{H}^{1}\left(\mathbb{R}^{N}\right)$. By definition, we have

$$
\tilde{\varepsilon}(0)=u_{0}-Q_{1 / \lambda}
$$


so that

$$
d \tilde{\varepsilon}=\psi+\frac{d \lambda}{\lambda(0)}(\Lambda Q)_{1 / \lambda(0)}
$$

Computation of $\boldsymbol{b}(\mathbf{0})$. From $(\mathrm{A}-6), b(0)$ is a $\mathscr{b}^{1}$ mapping with

$$
\begin{array}{r}
d b(0)=d \lambda\left[\frac{\left(\left(u_{1}\right)_{1 / \lambda(0)}, \chi_{M} \Phi\right)}{\left(\left(\Lambda u_{0}\right)_{1 / \lambda(0)}, \chi_{M} \Phi\right)}+\frac{\left(\left(\Lambda^{2} u_{0}\right)_{1 / \lambda(0)}, \chi_{M} \Phi\right)-\left(\left(\Lambda u_{1}\right)_{1 / \lambda(0)}, \chi_{M} \Phi\right)}{\left(\left(\Lambda u_{0}\right)_{1 / \lambda(0)}, \chi_{M} \Phi\right)^{2}}\right] \\
-\lambda(0) \frac{\left(\left(u_{1}\right)_{1 / \lambda(0)}, \chi_{M} \Phi\right)\left((\Lambda \psi)_{\left.1 / \lambda(0), \chi_{M} \Phi\right)}\right.}{\left(\left(\Lambda u_{0}\right)_{1 / \lambda(0)}, \chi_{M} \Phi\right)^{2}}
\end{array}
$$

where (A-6) and (A-7) ensure that, for some $d b \in \mathbb{R}$, we have

$$
d b(0)=d b+O\left(b_{0}\right)
$$

Computation of $\boldsymbol{\varepsilon}(\mathbf{0})$. Next,

$$
\varepsilon(0)=\tilde{\varepsilon}(0)-\left(P_{B_{1}(b(0))}-Q\right) .
$$

Consequently, $(\varepsilon(0), \psi)$ is also a smooth function of $d_{+}$with derivative $d p s_{1}(0)$ satisfying

$$
d p s_{1}(0)=(d \tilde{\varepsilon}, \psi)-d b(0)\left(\partial_{b} P_{B_{1}(b(0))}, \psi\right) .
$$

Replacing $d \tilde{\varepsilon}$ by its values, and applying that $(\Lambda Q, \psi)=0$ together with $|\lambda(0)-1| \lesssim b_{0}^{2} /\left|\log \left(b_{0}\right)\right|$, we get

$$
(d \tilde{\varepsilon}, \psi)=\|\psi\|_{L^{2}}^{2}+O\left(b_{0}\right)
$$

so that

$$
\operatorname{dps}_{1}(0)=\|\psi\|_{L^{2}}^{2}+O\left(b_{0}\right)
$$

Computation of $\partial_{s} \varepsilon(0)+b_{s}(0) \partial_{b} P_{B_{1}(b(0))}$. From (A-15),

$$
\partial_{s} \varepsilon(0)=-b_{s}(0) \partial_{b} P_{B_{1}(b(0))}-b(0) \Lambda u_{0}+\lambda(0)\left(b_{0} \Lambda P_{B_{1}\left(b_{0}\right)}\right)_{1 / \lambda(0)},
$$

so that $\left(\partial_{s} \varepsilon(0)+b_{s}(0) \partial_{b} P_{B_{1}(b(0))}, \psi\right)$ is a smooth function of $d_{+}$with derivative

$$
d p s_{2}(0)=-d b(0)\left(\Lambda u_{0}, \psi\right)+d \lambda\left(\left[\left(b_{0} \Lambda P_{B_{1}\left(b_{0}\right)}\right)_{1 / \lambda(0)}+\left(b_{0} \Lambda^{2} P_{B_{1}\left(b_{0}\right)}\right)_{1 / \lambda(0)}\right], \psi\right)-b(0)(\Lambda \psi, \psi),
$$

where, for the same orthogonality reason $(\Lambda Q, \psi)=0$, we have

$$
\left(\Lambda u_{0}, \psi\right)=(\Lambda Q, \psi)+O\left(b_{0}\right)=O\left(b_{0}\right)
$$

Consequently $d p s_{2}(0)=O\left(b_{0}\right)$. 
Conclusion. Finally, we have

$$
\kappa_{+}(0)=\frac{1}{2}\left[(\varepsilon(0), \psi)+\frac{1}{\sqrt{\zeta}}\left(\partial_{s} \varepsilon(0)+b_{s}(0) \partial_{b} P_{B_{1}(b(0))}, \psi\right)\right],
$$

and $\kappa_{+}(0)=a_{+}$reduces to a simple one-dimensional equation $F\left(d_{+}\right)=a_{+}$, with $F$ computed as combination of the above functions so that it is smooth in a neighborhood of 0 . Moreover,

$$
d F=\frac{1}{2}\left[d p s_{1}(0)+\frac{1}{\sqrt{\zeta}} d p s_{2}(0)\right]=\frac{1}{2}\|\psi\|_{L^{2}}^{2}+O\left(b_{0}\right),
$$

and (A-19) is proved. This concludes the proof of Lemma 3.3.

\section{Appendix B: Coercivity estimates}

The aim of this section is to prove the coercivity properties of the quadratic form

$$
B(\eta, \eta)=(\mathscr{B} v, v)=\int_{\mathbb{R}^{4}}\left|\partial_{r} \eta\right|^{2}+\int_{\mathbb{R}^{4}} W \eta^{2},
$$

where

$$
W(r)=2 V+\frac{3}{2} r V^{\prime}=\frac{6}{\left(1+r^{2} / 8\right)^{2}}-\frac{9}{4} \frac{r^{2}}{\left(1+r^{2} / 8\right)^{3}} .
$$

We use the elementary method developed in [Fibich et al. 2006]. The coercivity property of Lemma 4.7 is a consequence of the two following facts. First the index of $B$ on

$$
\dot{H}_{r}^{1}=\left\{u \text { radial }\left.\left|\int\right| \nabla u\right|^{2}+\int \frac{u^{2}}{r^{2}}<+\infty\right\}
$$

is at most 2. From standard Sturm-Liouville oscillation theorems, see Theorem XIII.8 [Reed and Simon 1978], this is equivalent to counting the number of zeroes of the solution to

$$
\left\{\begin{array}{l}
\mathscr{B} U=0, \\
U(0)=1, \quad U^{\prime}(0)=0,
\end{array}\right.
$$

on $(0, \infty)$, and this can be analytically reduced to counting the number of zeroes of a Bessel function. Then we need to show that the orthogonality conditions $(\eta, \psi)=(\eta, \Phi)=0$ are enough to treat the two negative directions. Arguing exactly as in [Fibich et al. 2006] — see also [Marzuola and Simpson 2011] — this is equivalent to first inverting the operator $\mathscr{B}$ on $\dot{H}_{\text {rad }}^{1}$, and then showing that $B$ restricted to $\operatorname{Span}\left\{\mathscr{B}^{-1} \psi, \mathscr{B}^{-1} \Phi\right\}$ is negative definite, which is an elementary numerical check. We shall check these two facts below and refer to [Fibich et al. 2006] for the proofs that this implies the claimed coercivity property. The proofs there are given for exponentially decaying functions and potentials, but one checks easily that the decay of the potential $|W(r)| \sim 1 / r^{4}$ at infinity and $|\Phi(r)| \sim 1 / r^{4}$ are more than enough to have all proofs go through. 
Computation of the index of $\boldsymbol{B}$. We first show that the index of $\mathscr{B}$ on $\dot{H}_{r}^{1}$ is at most 2 . We start by noting that $W(r) \geq \widehat{W}(r)$, where

$$
\hat{W}(r)=-\frac{3}{2} \frac{r^{2}}{\left(1+r^{2} / 8\right)^{3}} .
$$

Hence, classical Sturm-Liouville theory ensures that $U$ has less zeros than $\hat{U}$, the unique solution to

$$
-\frac{1}{r^{3}} \frac{d}{d r}\left[r^{3} \frac{d}{d r} \widehat{U}\right]+\widehat{W} \widehat{U}=0, \quad \widehat{U}(0)=1, \quad \widehat{U}^{\prime}(0)=0,
$$

on $(0, \infty)$. Second, we look for $\widehat{U}$ of the form $\widehat{U}(r)=\left(2 / r^{2}\right) \bar{U}\left(r^{2} / 2\right)$, with $\bar{U}$ a sufficiently smooth function. Denoting by $s$ the new variable $r^{2} / 2$, straightforward calculations yield that $\bar{U}$ is a solution to

$$
-\frac{d^{2}}{d s^{2}} \bar{U}+\bar{W} \bar{U}=0, \quad \bar{U}(0)=0, \quad \bar{U}^{\prime}(0)=1,
$$

on $(0, \infty)$, where

$$
\bar{W}(s)=-\frac{3}{2} \frac{1}{(1+s / 4)^{3}} .
$$

Setting then $\bar{U}(s)=\sqrt{1+s / 4} \widetilde{U}(1 / \sqrt{1+s / 4})$, we obtain that $\bar{U}$ is a solution to (B-3) if and only if $\widetilde{U}$ is a solution to

$$
\tau^{2} \frac{d^{2}}{d \tau^{2}} \widetilde{U}+\tau \frac{d}{d \tau} \widetilde{U}+\left(96 \tau^{2}-1\right) \widetilde{U}=0, \quad \widetilde{U}(1)=0, \quad \widetilde{U}^{\prime}(1)=-8,
$$

on $(0,1)$. Hence, $\widetilde{U}$ is a combination of Bessel functions: $\widetilde{U}(\tau)=C_{1} J(1,4 \sqrt{6} \tau)+C_{2} Y(1,4 \sqrt{6} \tau)$.

We compute $\left(C_{1}, C_{2}\right)$ and draw the explicit combination with Maple (Figure 1). The computed solution $\widetilde{U}$ has two zeros on $(0,1)$. Moreover, it diverges at 0 so that $\widetilde{U}(\tau) \sim K / \tau$ close to 0 with $K \neq 0$, As a

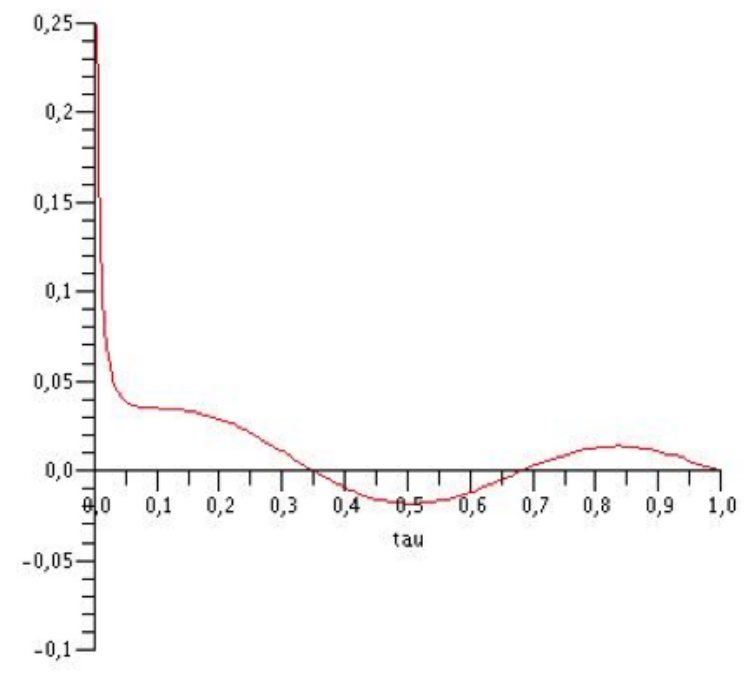

Figure 1. Solution to (B-3) computed by Maple. 


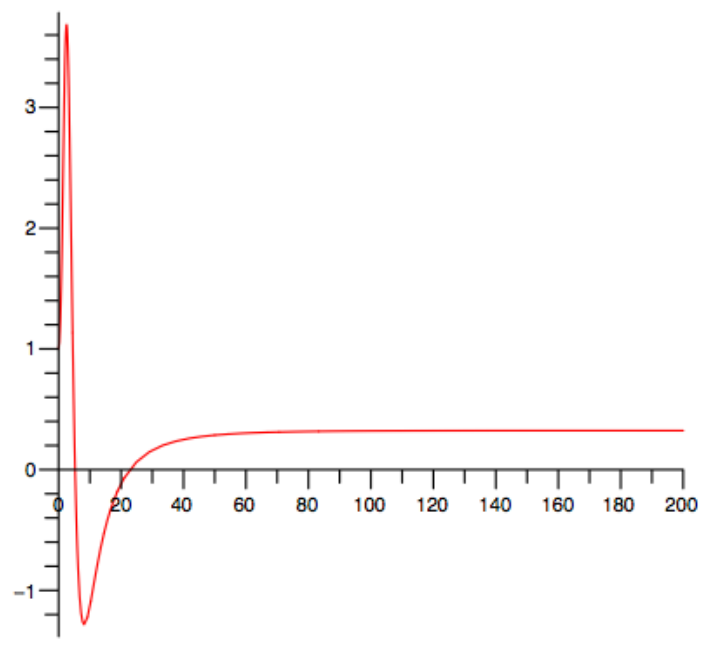

Figure 2. Solution to (B-1) computed by MAPLE.

consequence.

$$
\hat{U}(r) \sim \frac{1}{4} K \neq 0 \quad \text { when } r \rightarrow \infty
$$

and thus the index of $-\Delta+\hat{W}$ on $\dot{H}_{\text {rad }}^{1}$ is exactly two. Hence the index of $\mathscr{B}$ is at most 2.

Choice of the orthogonality conditions. We now invert $\mathscr{B}$. We first check numerically that the solution $U$ does not vanish at infinity, that is,

$$
\lim _{r \rightarrow+\infty} U(r)>0
$$

see Figure 2.

Hence $U$ is not a resonance - note that if $U$ had been a resonance, we could have removed the resonance by diminishing a bit the potential and getting a potential with index 2 and no resonance - and thus from standard ODE arguments [Fibich et al. 2006] there exists unique smooth solution in $\dot{H}^{1}{ }_{\text {rad }}$ of

$$
\mathscr{B} U=-\frac{1}{r^{3}} \frac{d}{d r}\left[r^{3} \frac{d}{d r} U\right]+W U=\psi, \quad U^{\prime}(0)=0,
$$

on $(0, \infty)$, with $\left(1+r^{2}\right) U \in L^{\infty}$, and

$$
\mathscr{B} U=-\frac{1}{r^{3}} \frac{d}{d r}\left[r^{3} \frac{d}{d r} U\right]+W U=\Phi, \quad U^{\prime}(0)=0,
$$

on $(0, \infty)$, with $\left(1+r^{2} / \log r\right) U \in L^{\infty}$. We denote by $\mathscr{B}^{-1} \psi$ and $\mathscr{B}^{-1} \Phi$ the respective solutions to these systems. We recall the explicit formula

$$
\Phi(r)=D \Lambda Q(r)=\frac{2-3 r^{2} / 4}{\left(1+r^{2} / 8\right)^{3}} .
$$


In the remainder of this section we check numerically that the restriction of $B$ to $\operatorname{Span}\left(\mathscr{B}^{-1} \psi, \mathscr{B}^{-1} \Phi\right)$ is negative definite, or equivalently:

Lemma. The symmetric matrix $\mathbb{B}=\left[\begin{array}{cc}\left(\mathscr{B}^{-1} \psi, \psi\right) & \left(\mathscr{B}^{-1} \Phi, \psi\right) \\ \left(\mathscr{B}^{-1} \Phi, \psi\right) & \left(\mathscr{B}^{-1} \Phi, \Phi\right)\end{array}\right]$ satisfies

$$
\left(\mathscr{B}^{-1} \psi, \psi\right)<0 \quad \text { and } \quad \operatorname{det} \mathbb{B}>0
$$

and is thus negative definite.

Numerical proof. We use standard MATLAB routines for the computation of solutions to (B-4) and (B-5). We note that we only fixed the initial value for $U^{\prime}(0)$. The value $U(0)$ is left open in order to achieve the expected decay at infinity that characterizes the inverse. To obtain $\mathscr{B}^{-1} \psi$, we first compute $\psi$. We obtain that the corresponding eigenvalue is approximately $l=-0.5860808922$. Because $\psi$ decays exponentially, we only need to obtain an approximation on a short time-range. We computed our solutions until $T_{\psi, \max }=30$. We emphasize here that we use an explicit scheme. As a drawback, the accumulation of errors tends to make the numerical solution become negative when the exact solution is exponentially small. Hence, our scheme becomes unstable after time $\widetilde{T}_{\psi, \max }=18$. Nevertheless, we extend our numerical solution by 0 after this time. This induces an exponentially small error. The pictures in Figure 3 illustrate this computation. On the left-hand side we draw the obtained solution. On the right-hand side, we draw $\psi_{\text {test }}(r)=\psi(r) \exp (\sqrt{-l} r)$. We observe here that our solution enters the exponential asymptotic regime before the instability comes into play.

The solution $\mathscr{B}^{-1} \psi$ is computed with the extension of $\psi$. Straightforward ODE analysis shows that the unique solution decaying fast at infinity behaves like $1 / r^{2}$ asymptotically. The choice of $U(0)$ is made with respect to this criterion. Figure 4 illustrates that we obtained a solution with the suitable decay. As previously, on the left-hand side is a picture of the numerical solution. On the right-hand side we plot $\mathscr{B}^{-1} \psi_{\text {test }}(r)=r^{2} \mathscr{B}^{-1} \psi(r)$. In the latter computations, this solution is involved in scalar products
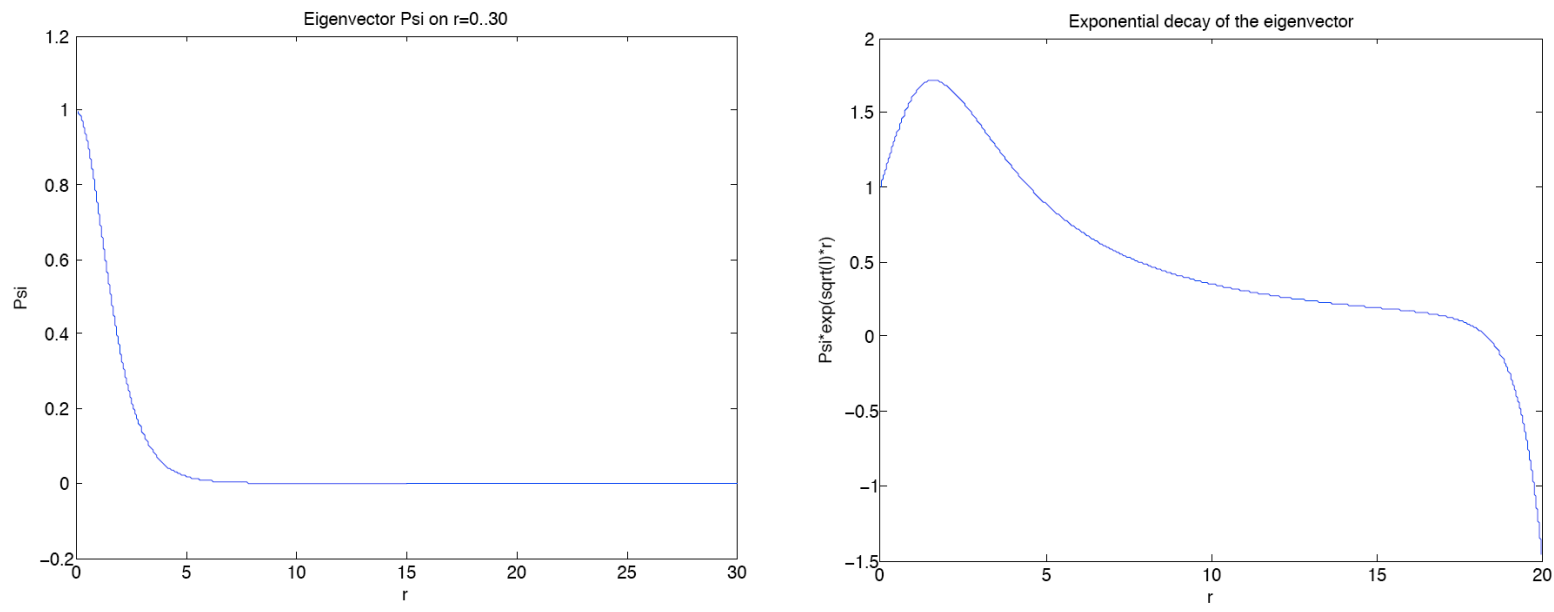

Figure 3. Numerical simulations for $\psi$. 

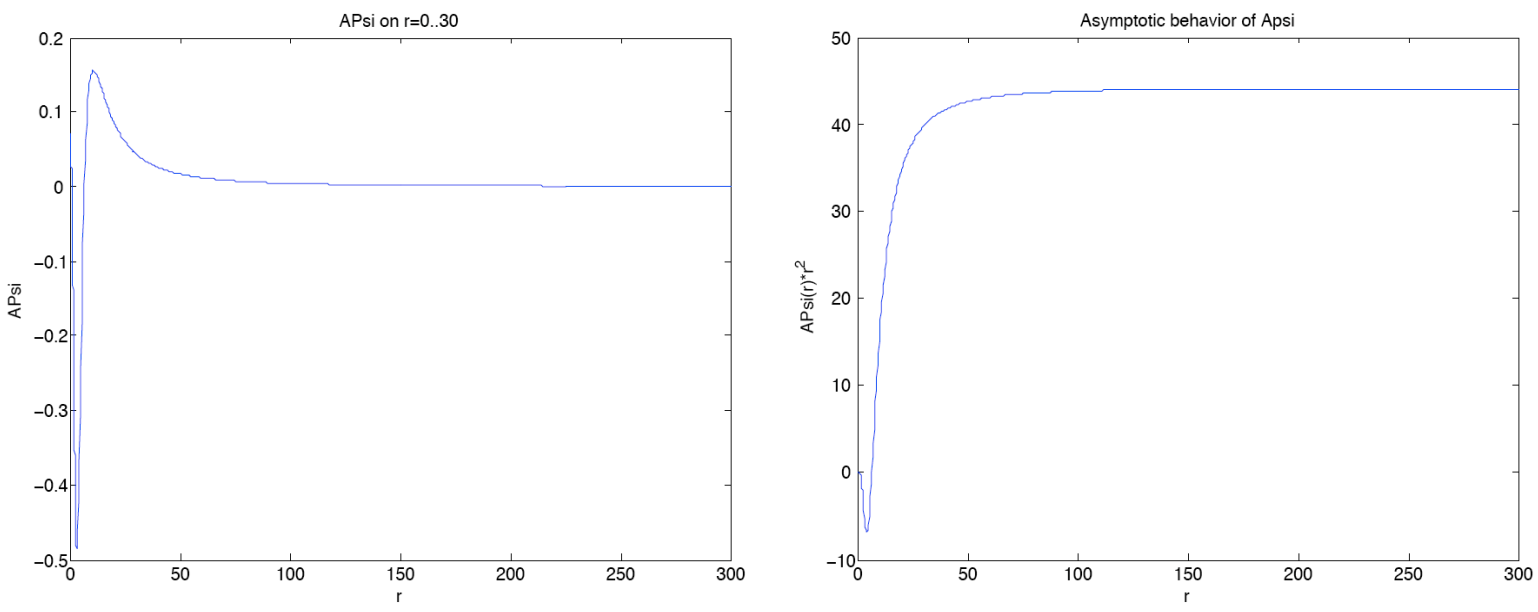

Figure 4. Numerical simulations for $\Re^{-1} \psi$.

with $\psi$. Hence even if drawn until $T_{\max }=300$, we only need a precise computation of this solution until $T_{\mathscr{B}^{-1} \psi, \max }=18$.

The last solution $\mathscr{B}^{-1} \Phi$ is computed with the same method. In this second case, the expected decay of the solution is $\log (r) / r^{2}$. Figure 5 illustrates that we obtained a solution with the suitable decay. The picture on the right-hand side restricts to the time-interval $r=0 \ldots 100$ because this is the significant region. In the latter computations, this solution is involved in integrals which converge slowly. Hence, we

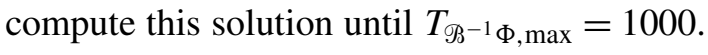

We now compute numerically the entries of the matrix $\mathbb{B}$. We first compute $\left(\mathscr{B}^{-1} \Phi, \psi\right)=\left(\mathscr{B}^{-1} \psi, \Phi\right)$. The exponential decay of $\psi$ implies that we need to compute the first integral $\left(\mathscr{B}^{-1} \Phi, \psi\right)$ on a shorter time-interval. Hence, we prefer this computation to the second one. We compute the $L^{2}$ scalar products with a standard trapezoidal method. Changing the time-interval and the time-step, the computations are
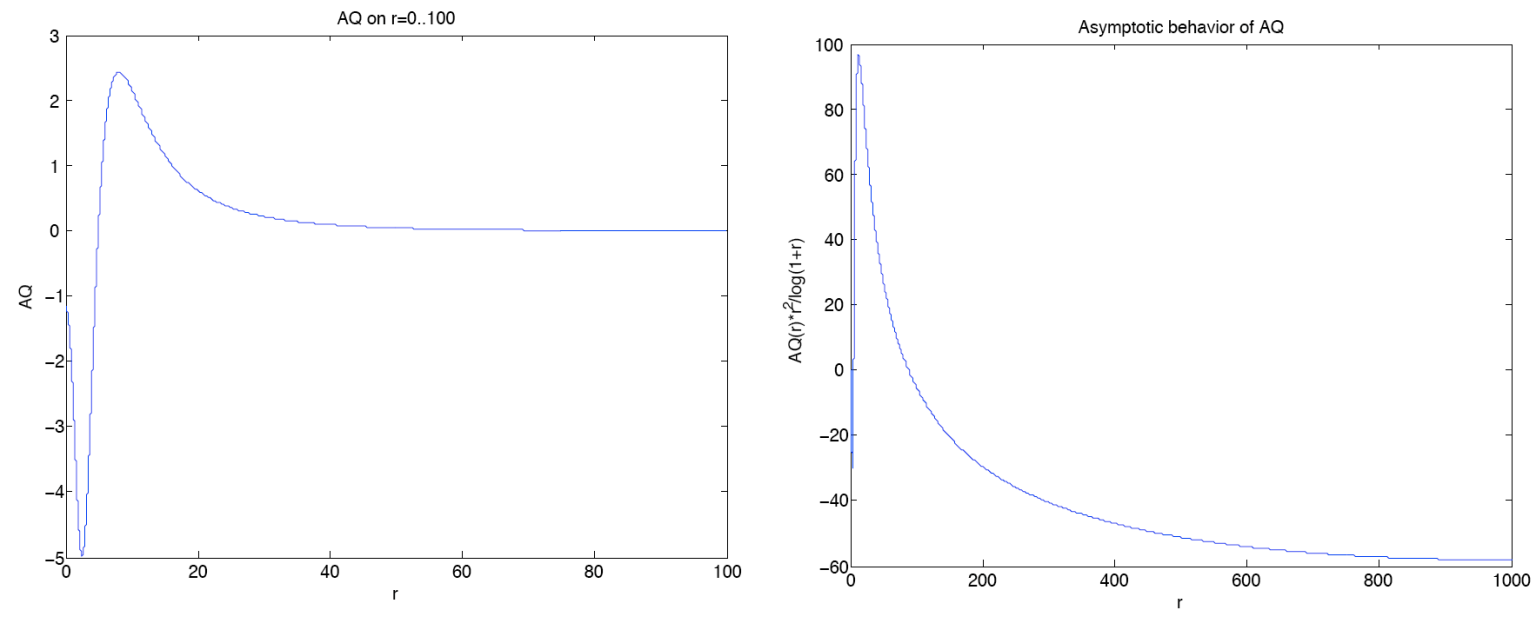

Figure 5. Numerical simulations for $\mathscr{B}^{-1} \Phi$. 


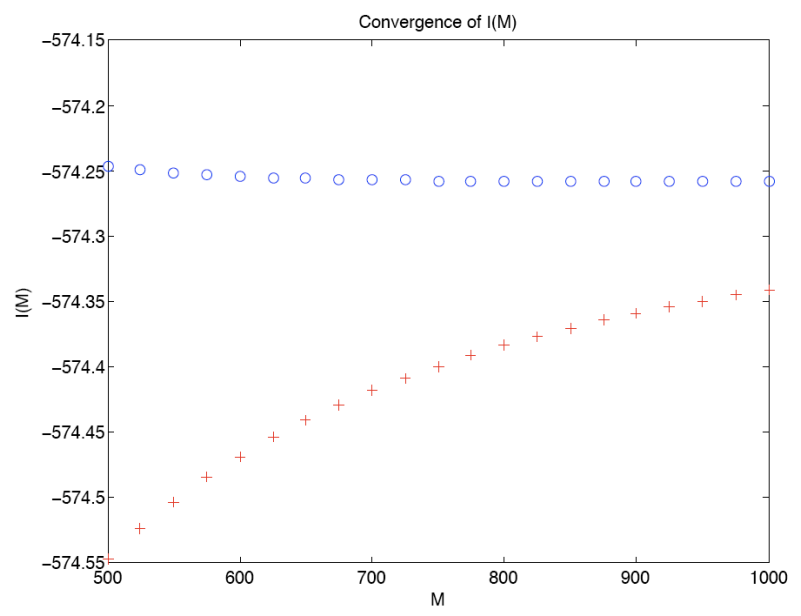

Figure 6. Computations for $\left(\Re^{-1} \Phi, \Phi\right)$.

stable up to an error of $10^{-2}$. We get the following approximations for the integrals involving $\psi$ :

$$
\left(\Re^{-1} \psi, \psi\right)=-4.63 \pm 10^{-2} \text { and }\left(\Re^{-1} \Phi, \psi\right)=32.65 \pm 10^{-2} .
$$

The last integral is a more involved computation. Indeed, standard real analysis implies that

$$
I(M):=\int_{0}^{M} \mathscr{B}^{-1} \Phi Q(r) \Phi(r) r^{3} \mathrm{~d} r=\left(\mathscr{B}^{-1} \Phi, \Phi\right)+\operatorname{err}(M),
$$

with a remainder satisfying $\operatorname{err}(M)=(K+o(1)) \ln (M) / M^{2}$ for some constant $K$. This remainder goes to 0 slowly; we see numerically that our computations have not converged even after integrating until $T_{\mathscr{B}^{-1}} \Phi, \max =1000$ (see Figure 6, red crosses). To improve the rate of convergence we compute an approximation of coefficient $K$ and subtract the estimated error term of our computations. This yields the blue circles in Figure 6. In this second computation we obtain a very good rate of convergence. Hence, we get the approximation $\left(\mathscr{B}^{-1} \Phi, \Phi\right)=-574.25 \pm 10^{-2}$, which leads to

$$
\operatorname{det}(\mathbb{B})=1591 \pm 10,
$$

concluding the numerical proof of the lemma.

\section{Appendix C: Some linear estimates}

We start by recalling some obvious integration-by-part results:

Lemma C.1. For any $N \geq 3$, there exists a constant $C$ for which there holds, for any $v \in H_{\mathrm{rad}}^{1}\left(\mathbb{R}^{N}\right)$,

$$
\left[\int_{\mathbb{R}^{N}} \frac{|v(y)|^{2}}{|y|^{2}}\right]^{1 / 2}+\sup _{y \in \mathbb{R}^{N}}\left(|y|^{(N-2) / 2}|v(y)|\right) \leq C\left[\int_{\mathbb{R}^{n}}|\nabla v(y)|^{2}\right]^{1 / 2} .
$$

Looking for control on further derivatives, we prove a lemma. 
Lemma C.2 (Hardy inequalities). Let $N=4$. Then for all $R>2$ and $v \in H_{\mathrm{rad}}^{2}\left(\mathbb{R}^{N}\right)$, we have

$$
\begin{gathered}
\int \frac{\left|\partial_{y} v\right|^{2}}{y^{2}} \lesssim \int(\Delta v)^{2} \\
\int_{y \leq R} \frac{|v|^{2}}{y^{4}(1+|\log y|)^{2}} \lesssim \int_{y \leq R} \frac{\left|\partial_{y} v\right|^{2}}{y^{2}}+\int_{y \leq 2}|v|^{2} \\
\int_{R \leq y \leq 2 R} \frac{|v|^{2}}{y^{4}} \lesssim \log R \int_{y \leq R} \frac{\left|\partial_{y} v\right|^{2}}{y^{2}}+\int_{y \leq 2}|v|^{2}
\end{gathered}
$$

Proof. Let $v$ be smooth. (C-2) follows from the explicit formula after integration by parts,

$$
\int(\Delta v)^{2}=\int\left(\partial_{y y} v+\frac{N-1}{y} \partial_{y} v\right)^{2}=\int\left(\partial_{y y} v\right)^{2}+(N-1) \int \frac{\left|\partial_{y} v\right|^{2}}{y^{2}}
$$

To prove (C-3), let $a \in[1,2]$ be such that

$$
|v(a)|^{2} \leq \int_{1 \leq y \leq 2}|v|^{2}
$$

Let $f(y)=-\left(1 / y^{3}(1+\log (y))\right) \mathbf{e}_{y}$ so that $\nabla \cdot f=1 /\left(y^{4}(1+|\log y|)^{2}\right)$, and integrate by parts to get

$$
\begin{aligned}
\int_{a \leq y \leq R} \frac{|v|^{2}}{y^{4}(1+\log y)^{2}} & =\int_{a \leq y \leq R}|v|^{2} \nabla \cdot f \\
& =-\left[\frac{|v|^{2}}{1+\log (y)}\right]_{a}^{R}+2 \int_{y \leq R} \frac{v \partial_{y} v}{y^{3}(1+\log y)} \\
& \lesssim|v(a)|^{2}+\left(\int_{y \leq R} \frac{|v|^{2}}{y^{4}(1+|\log y|)^{2}}\right)^{1 / 2}\left(\int_{y \leq R} \frac{\left|\partial_{y} v\right|^{2}}{y^{2}}\right)^{1 / 2} .
\end{aligned}
$$

similarly, using $\tilde{f}(y)=\left(1 / y^{3}(1-\log (y))\right) \mathbf{e}_{y}$, we get

$$
\begin{aligned}
\int_{\varepsilon \leq y \leq a} \frac{|v|^{2}}{y^{4}(1-\log y)^{2}} & =\int_{a \leq y \leq R}|v|^{2} \nabla \cdot \tilde{f} \\
& =\left[\frac{|v|^{2}}{1-\log (y)}\right]_{\varepsilon}^{a}+2 \int_{y \leq a} v \partial_{y} v \frac{1}{y^{3}(1-\log y)} \\
& \lesssim|v(a)|^{2}+\left(\int_{y \leq R} \frac{|v|^{2}}{y^{4}(1+|\log y|)^{2}}\right)^{1 / 2}\left(\int_{y \leq R} \frac{\left|\partial_{y} v\right|^{2}}{y^{2}}\right)^{1 / 2} .
\end{aligned}
$$

(C-5)-(C-7) now yield (C-3). The last inequality (C-4) is a straightforward variant of [Raphaël and Rodnianski 2012, Lemma B.1, (B.4)] and is left to the reader. 
Lemma C.3 (coercivity estimates with $H$ ). Let $\psi$ be the first eigenvector of $H$. Then there exist $c>0$ and $M_{0} \geq 1$ such that for $M \geq M_{0}$, there exists $\delta(M)>0$ such that given $u \in H_{\mathrm{rad}}^{1}\left(\mathbb{R}^{N}\right)$, we have

$$
\begin{aligned}
(H u, u) & \geq c \int\left(\partial_{y} u\right)^{2}-\frac{1}{c}\left[(u, \psi)^{2}+\left(u, \chi_{M} \Phi\right)^{2}\right], \\
\int(H u)^{2} & \geq \delta(M)\left[\int \frac{\left(\partial_{y} u\right)^{2}}{y^{2}}+\int \frac{u^{2}}{y^{4}(1+|\log y|)^{2}}\right]-\frac{1}{\delta(M)}\left(u, \chi_{M} \Phi\right)^{2} .
\end{aligned}
$$

Proof. (C-8) is a standard consequence of the coercivity of the linearized energy which admits exactly $\psi$ as bound state and $\Lambda Q$ as resonance at the origin, the good enough localization of $\Phi$ from (2-1) and the nondegeneracy from (2-2). The detailed proof is left to the reader.

To prove (C-9), we first observe the key subcoercivity property

$$
\begin{aligned}
\int(H u)^{2}=\int(\Delta u+V u)^{2} & =\int(\Delta u)^{2}-2 \int V\left(\partial_{y} u\right)^{2}+\int\left(\Delta V+V^{2}\right) u^{2} \\
& \geq c\left[\int(\Delta u)^{2}+\int \frac{u^{2}}{1+y^{6}}\right]-\frac{1}{c}\left[\int \frac{\left(\partial_{y} u\right)^{2}}{1+y^{4}}+\int \frac{u^{2}}{1+y^{8}}\right],
\end{aligned}
$$

where we used the asymptotic value

$$
V(y)=\frac{N(N+2)(N-2)}{y^{4}}\left[1+O\left(\frac{1}{y^{2}}\right)\right] \text { as } y \rightarrow+\infty .
$$

(C-9) now follows by contradiction. Let $M>0$ fixed and consider a sequence $u_{n}$ such that

$$
\int \frac{\left(\partial_{y} u_{n}\right)^{2}}{y^{2}}+\int \frac{u_{n}^{2}}{y^{4}(1+|\log y|)^{2}}=1
$$

and

$$
\int\left(H u_{n}\right)^{2} \leq \frac{1}{n}, \quad\left(u_{n}, \chi_{M} \Phi\right)=0 .
$$

Then by semicontinuity of the norm, a subsequence of $u_{n}$ weakly converges to a solution $u_{\infty} \in H_{\text {loc }}^{1}$ of $H u_{\infty}=0$. The solution $u_{\infty}$ is smooth away from the origin and hence the explicit integration of the ODE and the regularity assumption at the origin $u_{\infty} \in H_{\text {loc }}^{1}$ imply that

$$
u_{\infty}=\alpha \Lambda Q \text {. }
$$

On one hand, the uniform bound (C-11) together with the local compactness of Sobolev embeddings ensure that, up to a subsequence,

$$
\int \frac{\left(\partial_{y} u_{n}\right)^{2}}{1+y^{4}}+\int \frac{\left|u_{n}\right|^{2}}{1+y^{8}} \rightarrow \int \frac{\left(\partial_{y} u_{\infty}\right)^{2}}{1+y^{4}}+\int \frac{\left|u_{\infty}\right|^{2}}{1+y^{8}} \quad \text { and } \quad\left(u_{n}, \chi_{M} \Phi\right) \rightarrow\left(u_{\infty}, \chi_{M} \Phi\right),
$$

thanks to the $\chi_{M}$ localization. We thus conclude that

$$
\alpha\left(\Lambda Q, \chi_{M} \Phi\right)=\left(u_{\infty}, \chi_{M} \Phi\right)=0
$$

and thus $\alpha=0$. On the other hand, the subcoercivity property (C-10), the Hardy control (C-2), (C-3) and 
(C-11), (C-12) ensure that

$$
\int \frac{\left(\partial_{y} u_{n}\right)^{2}}{1+y^{4}}+\int \frac{u_{n}^{2}}{1+y^{8}} \geq C>0
$$

from which

$$
\alpha^{2}\left[\int \frac{\left(\partial_{y} \Lambda Q\right)^{2}}{1+y^{4}}+\int \frac{|\Lambda Q|^{2}}{1+y^{8}}\right]=\int \frac{\left(\partial_{y} u_{\infty}\right)^{2}}{1+y^{4}}+\int \frac{\left|u_{\infty}\right|^{2}}{1+y^{8}} \geq C>0,
$$

and thus $\alpha \neq 0$. A contradiction follows. This concludes the proof of (C-9) and of Lemma C.3.

Straightforward computations show that the coercivity estimates with $H$ can be adapted to any of the operators $H_{\lambda}$ yielding, for any $\lambda>0$ and $u \in H_{\text {rad }}^{1}\left(\mathbb{R}^{N}\right)$,

$$
\left(H_{\lambda} u, u\right) \geq c \int\left(\partial_{y} u\right)^{2}-\frac{1}{c \lambda^{4}}\left[\left(u,(\psi)_{\lambda}\right)^{2}+\left(u,\left(\chi_{M} \Phi\right)_{\lambda}\right)^{2}\right]
$$

for the same $c$ and $\delta(M)$ as in Lemma C.3.

\section{Acknowledgements}

The authors thank Igor Rodnianski for stimulating discussions about this work. P. Raphaël is supported by the French ANR Jeune Chercheur SWAP.

\section{References}

[Alinhac 1995] S. Alinhac, Blowup for nonlinear hyperbolic equations, Progress in nonlinear differential equations and their applications 17, Birkhäuser, Boston, MA, 1995. MR 1339762 Zbl 0820.35001

[van den Berg et al. 2003] J. B. van den Berg, J. Hulshof, and J. R. King, "Formal asymptotics of bubbling in the harmonic map heat flow", SIAM J. Appl. Math. 63:5 (2003), 1682-1717. MR 2004h:35097 Zbl 1037.35023

[Bizoń et al. 2001] P. Bizoń, T. Chmaj, and Z. Tabor, "Formation of singularities for equivariant $(2+1)$-dimensional wave maps into the 2-sphere", Nonlinearity 14:5 (2001), 1041-1053. MR 2003b:58043 Zbl 0988.35010

[Christodoulou and Tahvildar-Zadeh 1993] D. Christodoulou and A. S. Tahvildar-Zadeh, "On the regularity of spherically symmetric wave maps", Comm. Pure Appl. Math. 46:7 (1993), 1041-1091. MR 94e:58030 Zbl 0744.58071

[Cote et al. 2009] R. Cote, Y. Martel, and F. Merle, "Construction of multisolitons solutions for the $L^{2}$-supercritical gKdV and NLS equations", preprint, 2009. arXiv 0905.0470

[Duyckaerts et al. 2011] T. Duyckaerts, C. Kenig, and F. Merle, "Universality of blow-up profile for small radial type II blow-up solutions of the energy-critical wave equation”, J. Eur. Math. Soc. (JEMS) 13:3 (2011), 533-599. MR 2012e:35160 Zbl 1230.35067

[Duyckaerts et al. 2012] T. Duyckaerts, C. Kenig, and F. Merle, "Universality of the blow-up profile for small type II blow-up solutions of the energy-critical wave equation: the nonradial case", J. Eur. Math. Soc. (JEMS) 14:5 (2012), 1389-1454. MR 2966655

[Fibich et al. 2006] G. Fibich, F. Merle, and P. Raphaël, "Proof of a spectral property related to the singularity formation for the $L^{2}$ critical nonlinear Schrödinger equation”, Phys. D 220:1 (2006), 1-13. MR 2007d:35254 Zbl 1100.35097

[Grillakis 1990] M. G. Grillakis, "Regularity and asymptotic behaviour of the wave equation with a critical nonlinearity", Ann. of Math. (2) 132:3 (1990), 485-509. MR 92c:35080 Zbl 0736.35067

[Jörgens 1961] K. Jörgens, "Das Anfangswertproblem im Grossen für eine Klasse nichtlinearer Wellengleichungen”, Math. Z. 77 (1961), 295-308. MR 24 \#A323 Zbl 0111.09105

[Karageorgis and Strauss 2007] P. Karageorgis and W. A. Strauss, "Instability of steady states for nonlinear wave and heat equations", J. Differential Equations 241:1 (2007), 184-205. MR 2009a:35118 Zbl 1130.35015 
[Kavian and Weissler 1990] O. Kavian and F. B. Weissler, "Finite energy self-similar solutions of a nonlinear wave equation", Comm. Partial Differential Equations 15:10 (1990), 1381-1420. MR 91m:35151 Zbl 0726.35085

[Kenig and Merle 2008] C. E. Kenig and F. Merle, "Global well-posedness, scattering and blow-up for the energy-critical focusing non-linear wave equation”, Acta Math. 201:2 (2008), 147-212. MR 2011a:35344 Zbl 1183.35202

[Krieger and Schlag 2007] J. Krieger and W. Schlag, "On the focusing critical semi-linear wave equation", Amer. J. Math. 129:3 (2007), 843-913. MR 2009f:35231 Zbl 1219.35144

[Krieger and Schlag 2009] J. Krieger and W. Schlag, "Non-generic blow-up solutions for the critical focusing NLS in 1-D", J. Eur. Math. Soc. (JEMS) 11:1 (2009), 1-125. MR 2009m:35488 Zbl 1163.35035

[Krieger et al. 2008] J. Krieger, W. Schlag, and D. Tataru, "Renormalization and blow up for charge one equivariant critical wave maps", Invent. Math. 171:3 (2008), 543-615. MR 2009b:58061 Zbl 1139.35021

[Krieger et al. 2009a] J. Krieger, W. Schlag, and D. Tataru, "Renormalization and blow up for the critical Yang-Mills problem", Adv. Math. 221:5 (2009), 1445-1521. MR 2010h:58023 Zbl 1183.35203

[Krieger et al. 2009b] J. Krieger, W. Schlag, and D. Tataru, "Slow blow-up solutions for the $H^{1}\left(\mathbb{R}^{3}\right)$ critical focusing semilinear wave equation”, Duke Math. J. 147:1 (2009), 1-53. MR 2010h:58045 Zbl 1170.35066

[Levine 1974] H. A. Levine, "Instability and nonexistence of global solutions to nonlinear wave equations of the form $P u_{t t}=-A u+\mathscr{F}(u) "$, Trans. Amer. Math. Soc. 192 (1974), 1-21. MR 49 \#9436 Zbl 0288.35003

[Martel 2005] Y. Martel, "Asymptotic $N$-soliton-like solutions of the subcritical and critical generalized Korteweg-de Vries equations", Amer. J. Math. 127:5 (2005), 1103-1140. MR 2007a:35128 Zbl 1090.35158

[Martel and Merle 2002] Y. Martel and F. Merle, "Stability of blow-up profile and lower bounds for blow-up rate for the critical generalized KdV equation", Ann. of Math. (2) 155:1 (2002), 235-280. MR 2003e:35270 Zbl 1005.35081

[Marzuola and Simpson 2011] J. L. Marzuola and G. Simpson, "Spectral analysis for matrix Hamiltonian operators", Nonlinearity 24:2 (2011), 389-429. MR 2012b:35328 Zbl 1213.35371

[Merle and Raphael 2003] F. Merle and P. Raphael, "Sharp upper bound on the blow-up rate for the critical nonlinear Schrödinger equation”, Geom. Funct. Anal. 13:3 (2003), 591-642. MR 2005j:35207 Zbl 1061.35135

[Merle and Raphael 2004] F. Merle and P. Raphael, "On universality of blow-up profile for $L^{2}$ critical nonlinear Schrödinger equation”, Invent. Math. 156:3 (2004), 565-672. MR 2006a:35283 Zbl 1067.35110

[Merle and Raphael 2005a] F. Merle and P. Raphael, "The blow-up dynamic and upper bound on the blow-up rate for critical nonlinear Schrödinger equation”, Ann. of Math. (2) 161:1 (2005), 157-222. MR 2006k:35277 Zbl 1185.35263

[Merle and Raphael 2005b] F. Merle and P. Raphael, "Profiles and quantization of the blow up mass for critical nonlinear Schrödinger equation”, Comm. Math. Phys. 253:3 (2005), 675-704. MR 2006m:35346 Zbl 1062.35137

[Merle and Raphael 2006] F. Merle and P. Raphael, "On a sharp lower bound on the blow-up rate for the $L^{2}$ critical nonlinear Schrödinger equation”, J. Amer. Math. Soc. 19:1 (2006), 37-90. MR 2006j:35223 Zbl 1075.35077

[Merle and Zaag 2003] F. Merle and H. Zaag, "Determination of the blow-up rate for the semilinear wave equation", Amer. J. Math. 125:5 (2003), 1147-1164. MR 2004g:35163 Zbl 1052.35043

[Merle and Zaag 2008] F. Merle and H. Zaag, "Openness of the set of non-characteristic points and regularity of the blow-up curve for the 1 D semilinear wave equation”, Comm. Math. Phys. 282:1 (2008), 55-86. MR 2010e:35193 Zbl 1159.35046

[Raphael 2005] P. Raphael, "Stability of the log-log bound for blow up solutions to the critical non linear Schrödinger equation", Math. Ann. 331:3 (2005), 577-609. MR 2006b:35303 Zbl 1082.35143

[Raphäl and Rodnianski 2012] P. Raphaël and I. Rodnianski, "Stable blow up dynamics for the critical co-rotational wave maps and equivariant Yang-Mills problems", Publ. Math. Inst. Hautes Études Sci. 115:1 (2012), 1-122. MR 2929728

[Raphaël and Szeftel 2011] P. Raphaël and J. Szeftel, "Existence and uniqueness of minimal blow-up solutions to an inhomogeneous mass critical NLS", J. Amer. Math. Soc. 24:2 (2011), 471-546. MR 2748399 Zbl 1218.35226

[Reed and Simon 1978] M. Reed and B. Simon, Methods of modern mathematical physics, vol. 4: Analysis of operators, Academic Press, New York, 1978. MR 0493421 Zbl 0401.47001

[Rodnianski and Sterbenz 2010] I. Rodnianski and J. Sterbenz, "On the formation of singularities in the critical O(3) $\sigma$-model", Ann. of Math. (2) 172:1 (2010), 187-242. MR 2011i:58023 Zbl 1213.35392 
[Shatah and Tahvildar-Zadeh 1994] J. Shatah and A. S. Tahvildar-Zadeh, "On the Cauchy problem for equivariant wave maps", Comm. Pure Appl. Math. 47:5 (1994), 719-754. MR 96c:58049 Zbl 0811.58059

[Sogge 1995] C. D. Sogge, Lectures on nonlinear wave equations, Monographs in Analysis 2, International Press, Boston, MA, 1995. MR 2000g:35153 Zbl 1089.35500

[Strauss 1989] W. A. Strauss, Nonlinear wave equations, CBMS Regional Conference Series in Mathematics 73, American Mathematical Society, Providence, RI, 1989. MR 91g:35002 Zbl 0714.35003

[Struwe 1988] M. Struwe, "Globally regular solutions to the $u^{5}$ Klein-Gordon equation", Ann. Scuola Norm. Sup. Pisa Cl. Sci. (4) 15:3 (1988), 495-513. MR 90j:35142 Zbl 0728.35072

[Struwe 2003] M. Struwe, "Equivariant wave maps in two space dimensions", Comm. Pure Appl. Math. 56:7 (2003), 815-823. MR 2004c:58061 Zbl 1033.53019

Received 8 Oct 2010. Accepted 13 May 2011.

MatthiEU HiLlairet: hillairet@ceremade.dauphine.fr Ceremade, Université Paris Dauphine, 75775 Paris Cedex 16, France http://www.ceremade.dauphine.fr/ mhillair/

PIERRE RAPHAËL: pierre.raphael@math.univ-toulouse.fr Université Paul Sabatier, Institut de Mathématiques de Toulouse, 31062 Toulouse Cedex 9, France http://www.math.univ-toulouse.fr/ raphael/ 


\title{
Analysis \& PDE
}

\author{
msp.berkeley.edu/apde
}

EDITORS

EDITOR-IN-CHIEF

Maciej Zworski

University of California

Berkeley, USA

BOARD OF EDITORS

\begin{tabular}{|c|c|c|c|}
\hline Michael Aizenman & $\begin{array}{l}\text { Princeton University, USA } \\
\text { aizenman@math.princeton.edu }\end{array}$ & Nicolas Burq & $\begin{array}{l}\text { Université Paris-Sud 11, France } \\
\text { nicolas.burq@math.u-psud.fr }\end{array}$ \\
\hline Luis A. Caffarelli & $\begin{array}{l}\text { University of Texas, USA } \\
\text { caffarel@math.utexas.edu }\end{array}$ & un-Yung Alice Chang & $\begin{array}{l}\text { Princeton University, USA } \\
\text { chang@math.princeton.edu }\end{array}$ \\
\hline Michael Christ & $\begin{array}{l}\text { University of California, Berkeley, USA } \\
\text { mchrist@math.berkeley.edu }\end{array}$ & Charles Fefferman & $\begin{array}{l}\text { Princeton University, USA } \\
\text { cf@math.princeton.edu }\end{array}$ \\
\hline Ursula Hamenstaedt & $\begin{array}{l}\text { Universität Bonn, Germany } \\
\text { ursula@math.uni-bonn.de }\end{array}$ & Nigel Higson & $\begin{array}{l}\text { Pennsylvania State Univesity, USA } \\
\text { higson@ math.psu.edu }\end{array}$ \\
\hline Vaughan Jones & $\begin{array}{l}\text { University of California, Berkeley, USA } \\
\text { vfr@math.berkeley.edu }\end{array}$ & Herbert Koch & $\begin{array}{l}\text { Universität Bonn, Germany } \\
\text { koch@math.uni-bonn.de }\end{array}$ \\
\hline Izabella Laba & $\begin{array}{l}\text { University of British Columbia, Canada } \\
\text { ilaba@math.ubc.ca }\end{array}$ & Gilles Lebeau & $\begin{array}{l}\text { Université de Nice Sophia Antipolis, France } \\
\text { lebeau@unice.fr }\end{array}$ \\
\hline László Lempert & $\begin{array}{l}\text { Purdue University, USA } \\
\text { lempert@math.purdue.edu }\end{array}$ & Richard B. Melrose & $\begin{array}{l}\text { Massachussets Institute of Technology, USA } \\
\text { rbm@math.mit.edu }\end{array}$ \\
\hline Frank Merle & $\begin{array}{l}\text { Université de Cergy-Pontoise, France } \\
\text { Frank.Merle@u-cergy.fr }\end{array}$ & William Minicozzi II & $\begin{array}{l}\text { Johns Hopkins University, USA } \\
\text { minicozz@ math.jhu.edu }\end{array}$ \\
\hline Werner Müller & $\begin{array}{l}\text { Universität Bonn, Germany } \\
\text { mueller@math.uni-bonn.de }\end{array}$ & Yuval Peres & $\begin{array}{l}\text { University of California, Berkeley, USA } \\
\text { peres@stat.berkeley.edu }\end{array}$ \\
\hline Gilles Pisier & $\begin{array}{l}\text { Texas A\&M University, and Paris } 6 \\
\text { pisier@math.tamu.edu }\end{array}$ & Tristan Rivière & $\begin{array}{l}\text { ETH, Switzerland } \\
\text { riviere@math.ethz.ch }\end{array}$ \\
\hline Igor Rodnianski & $\begin{array}{l}\text { Princeton University, USA } \\
\text { irod@math.princeton.edu }\end{array}$ & Wilhelm Schlag & $\begin{array}{l}\text { University of Chicago, USA } \\
\text { schlag@math.uchicago.edu }\end{array}$ \\
\hline Sylvia Serfaty & $\begin{array}{l}\text { New York University, USA } \\
\text { serfaty@ cims.nyu.edu }\end{array}$ & Yum-Tong Siu & $\begin{array}{l}\text { Harvard University, USA } \\
\text { siu@math.harvard.edu }\end{array}$ \\
\hline Terence Tao & $\begin{array}{l}\text { University of California, Los Angeles, USA } \\
\text { tao@math.ucla.edu }\end{array}$ & A Michael E. Taylor & $\begin{array}{l}\text { Univ. of North Carolina, Chapel Hill, USA } \\
\text { met@math.unc.edu }\end{array}$ \\
\hline Gunther Uhlmann & $\begin{array}{l}\text { University of Washington, USA } \\
\text { gunther@math.washington.edu }\end{array}$ & András Vasy & $\begin{array}{l}\text { Stanford University, USA } \\
\text { andras@math.stanford.edu }\end{array}$ \\
\hline Virgil Voiculescu & $\begin{array}{l}\text { University of California, Berkeley, USA } \\
\text { dvv@math.berkeley.edu }\end{array}$ & Steven Zelditch & $\begin{array}{l}\text { Northwestern University, USA } \\
\text { zelditch@math.northwestern.edu }\end{array}$ \\
\hline
\end{tabular}

\section{PRODUCTION}

contact@msp.org

Silvio Levy, Scientific Editor

Sheila Newbery, Senior Production Editor

See inside back cover or msp.berkeley.edu/apde for submission instructions.

The subscription price for 2012 is US \$140/year for the electronic version, and \$240/year for print and electronic. Subscriptions, requests for back issues from the last three years and changes of subscribers address should be sent to Mathematical Sciences Publishers, Department of Mathematics, University of California, Berkeley, CA 94720-3840, USA.

Analysis \& PDE, at Mathematical Sciences Publishers, Department of Mathematics, University of California, Berkeley, CA 94720-3840 is published continuously online. Periodical rate postage paid at Berkeley, CA 94704, and additional mailing offices.

APDE peer review and production are managed by EditFLOW ${ }^{\mathrm{TM}}$ from Mathematical Sciences Publishers.

PUBLISHED BY

mathematical sciences publishers

http://msp.org/

A NON-PROFIT CORPORATION

Typeset in IATEX

Copyright $(2012$ by Mathematical Sciences Publishers 


\section{ANALYSIS \& PDE}

\section{Volume $5 \quad$ No. $4 \quad 2012$}

On the global well-posedness of energy-critical Schrödinger equations in curved spaces

Alexandru D. IONESCU, BenOIT PAusader and Gigliola StafFilani

Generalized Ricci flow, I: Higher-derivative estimates for compact manifolds

YI LI

Smooth type II blow-up solutions to the four-dimensional energy-critical wave equation

Matthieu Hillairet and PierRe RaphaËL

Nonconcentration in partially rectangular billiards

LUC Hillairet and JEREMY L. MARZUOLA

Global well-posedness and scattering for the defocusing quintic NLS in three dimensions ROWAN KILLIP and MONICA VIŞAN 